Supporting Information

\title{
Enthalpy-Controlled Insertion of a 'Nonspectator' Tricoordinate Phosphorus Ligand into Group 10 Transition Metal-Carbon Bonds
}

Seung Jun Hwang, ${ }^{\# \dagger}$ Akira Tanushi, ${ }^{\# \dagger}$ and Alexander T. Radosevich* ${ }^{\dagger}$

${ }^{\dagger}$ Department of Chemistry Massachusetts Institute of Technology, Cambridge, Massachusetts, 02139, United States

I| Present Address: Department of Chemistry, Pohang University of Science and Technology (POSTECH), Pohang, 37673, Republic of Korea

${ }^{\perp}$ These authors contributed equally to this work.

Correspondence to: radosevich@mit.edu 


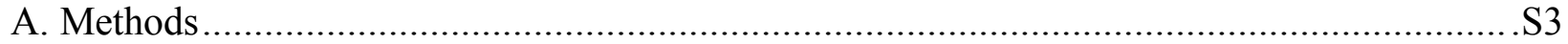

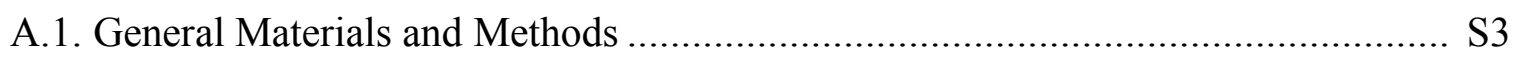

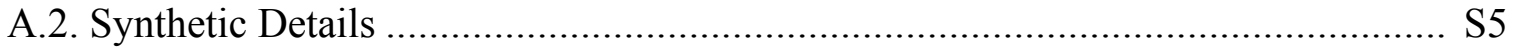

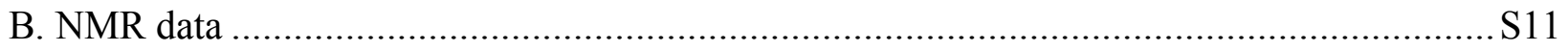

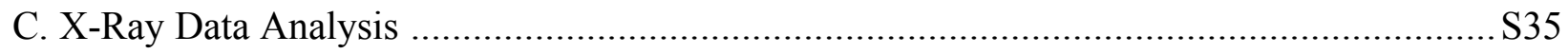

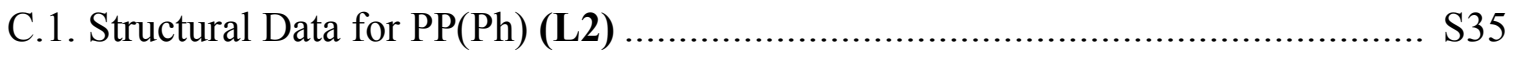

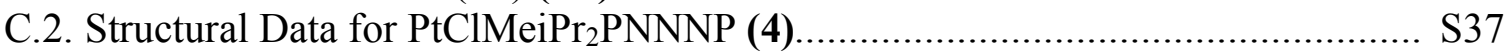

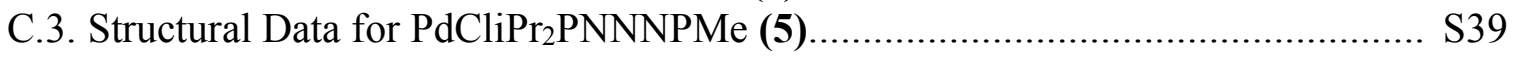

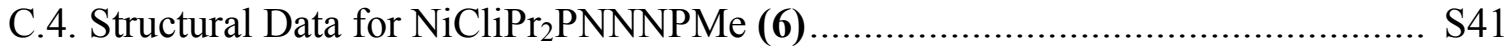

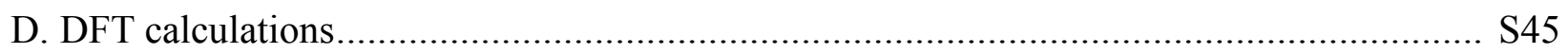

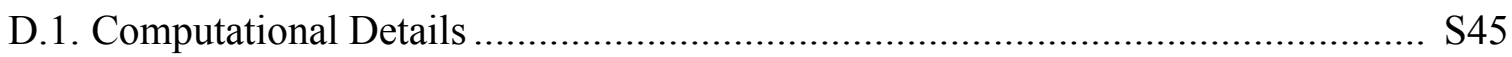

D.2. Comparison of Crystallographic and Computed Structures of 4, 5 and $\mathbf{6} \ldots \ldots \ldots \ldots . . .546$

D.3. Energies and XYZ Coordinates.................................................................... S47

D.4. Energies and XYZ Coordinates..................................................................... S47

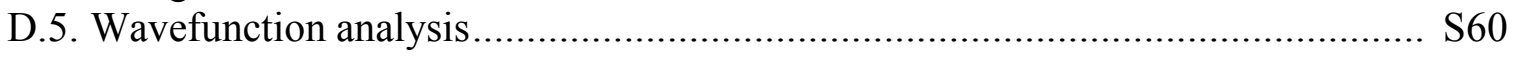

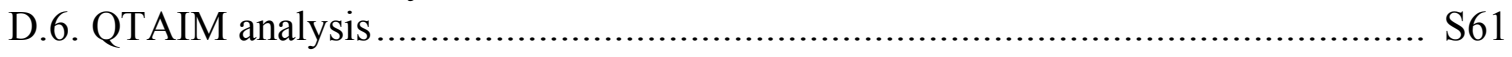

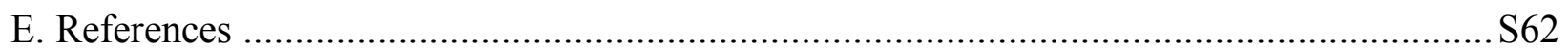




\section{A. Methods}

\section{A.1. General Materials and Methods}

All reactions were carried out on the benchtop inside a fume hood. $\mathrm{Pd}_{2} \mathrm{dba}_{3}$, (cod) $\mathrm{Pd}(\mathrm{Me})(\mathrm{Cl})$, (cod)Pt(Me), Xantphos, DiPPF, and diisopropylphosphine were obtained from Strem Chemical. Sodium tert-butoxide, triethylamine, palladium(II) acetate, bromoiodobenzene, iodobenzene, diphenylphosphine, phosphorus trichloride, $10 \mathrm{wt} \%$ palladium on carbon, and solvents were obtained from Sigma Aldrich. All chemicals were used without further purification. Bis(2aminophenyl)amine ${ }^{1}(\mathrm{cod}) \mathrm{Pt}(\mathrm{Me})(\mathrm{Cl})^{2}$, and $\mathrm{Ni}(\mathrm{Me})(\mathrm{Cl})\left(\mathrm{PMe}_{3}\right)_{2}{ }^{3}$ were prepared as previously reported. Cross couplings were carried out in sealed heavy-walled reaction flasks (Chemglass Part \# CG-1880-R-03) fitted with perfluoro O-rings (Chemglass part \#CG-309-220). Column chromatography was carried out on silica gel (SiliFlash ${ }^{\circledR}$ Irregular Silica Gel, P60 40-63 $\mu$ m). Thin layer chromatography was performed using Analtech aluminum backed fluorescent silica gel (UV254). All reactions were heated in temperature controlled silicone oil baths where the oil level reached at least $3 / 4$ of the reaction volume at any time. All yields are isolated yields of purified products. All metalation chemistry and handling of the resulting compounds was performed in a VAC-Atmospheres Nexus II nitrogen filled glovebox. All solvents in the glove box were taken from a Glass Contour Solvent Purification System utilizing alumina columns and solvents degassed/stored under argon gas. All vials and pipettes in the glovebox were heated to $120^{\circ} \mathrm{C}$ for a minimum of $24 \mathrm{~h}$ in an oven before being brought into the glovebox.

NMR spectra were recorded on a Varian Inova-500 (500 MHz) spectrometer at the MIT Department of Chemistry Instrumentation Facility and processed with a MestReNova software. ${ }^{1} \mathrm{H}$ and ${ }^{13} \mathrm{C}\left\{{ }^{1} \mathrm{H}\right\}$ NMR spectra were internally referenced to the residual solvent signal $(\delta=7.26$ and 77.16 ppm for $\mathrm{CHCl}_{3}$ in $\mathrm{CDCl}_{3}-\mathrm{d}_{1}$ respectively), ${ }^{4}$ while ${ }^{31} \mathrm{P}\left\{{ }^{1} \mathrm{H}\right\}$ NMR spectra were externally referenced to $85 \% \mathrm{H}_{3} \mathrm{PO}_{4}$. Multiplicities are described as $\mathrm{s}=$ singlet, brs $=$ broad singlet, $\mathrm{d}=$ doublet, $\mathrm{t}=$ triplet, $\mathrm{q}=$ quartet, $\mathrm{dd}=$ doublet of doublets, $\mathrm{td}=$ triplet of doublets, $\mathrm{m}=$ multiplet. Coupling constants are reported as $J$-values in Hz. High-resolution mass spectra were obtained at the Mass Spectrometry Laboratory in the Department of Chemistry Instrumentation Facility, MIT, using either Agilent QTOF 6545 with ESI ionization source or a JEOL AccuTOF-DART (JMST100LP, ionSense DART source).

X-ray structures of compounds $\mathbf{L} 2$ and $\mathbf{6}^{\prime}$ data were collected on a Bruker three-circle platform goniometer equipped with an Apex II CCD and an Oxford cryostream cooling device operating at $100 \mathrm{~K}$. Radiation was from a graphite fine focus sealed tube Mo K $\alpha(0.71073 \AA)$ source. Crystals were mounted on a glass fiber pin using Paratone $\mathrm{N}$ oil. Data was collected as a series of $\varphi$ and/or $\omega$ scans. Data was integrated using SAINT and scaled with multi-scan absorption correction using SADABS. ${ }^{5}$ The structures were solved by intrinsic phasing using SHELXT (Apex3 program suite v2016.1.0) and refined against $\mathrm{F}^{2}$ on all data by full matrix least squares with SHELXL-97. ${ }^{6}$ All non-hydrogen atoms were refined anisotropically. $\mathrm{H}$ atoms were placed at idealized positions and refined using a riding model. 
X-ray structures of complexes $\mathbf{4}$ and $\mathbf{5}^{\prime}$ were collected using $0.24797 \AA$ radiation at temperature of $15 \mathrm{~K}$ (Oxford Diffraction Helijet) on a vertical mounted Bruker D8 three-circle platform goniometer equipped with an Apex II CCD at ChemMatCARS located at Advances Photon Source (APS), Argonne National Laboratory (ANL). Structures were solved and refined as described above. Crystal data and refinement statistics are summarized in Tables S1-S4 and thermal ellipsoid plots are collected in Figs. S26-S29. 


\section{A.2. Synthetic Details}

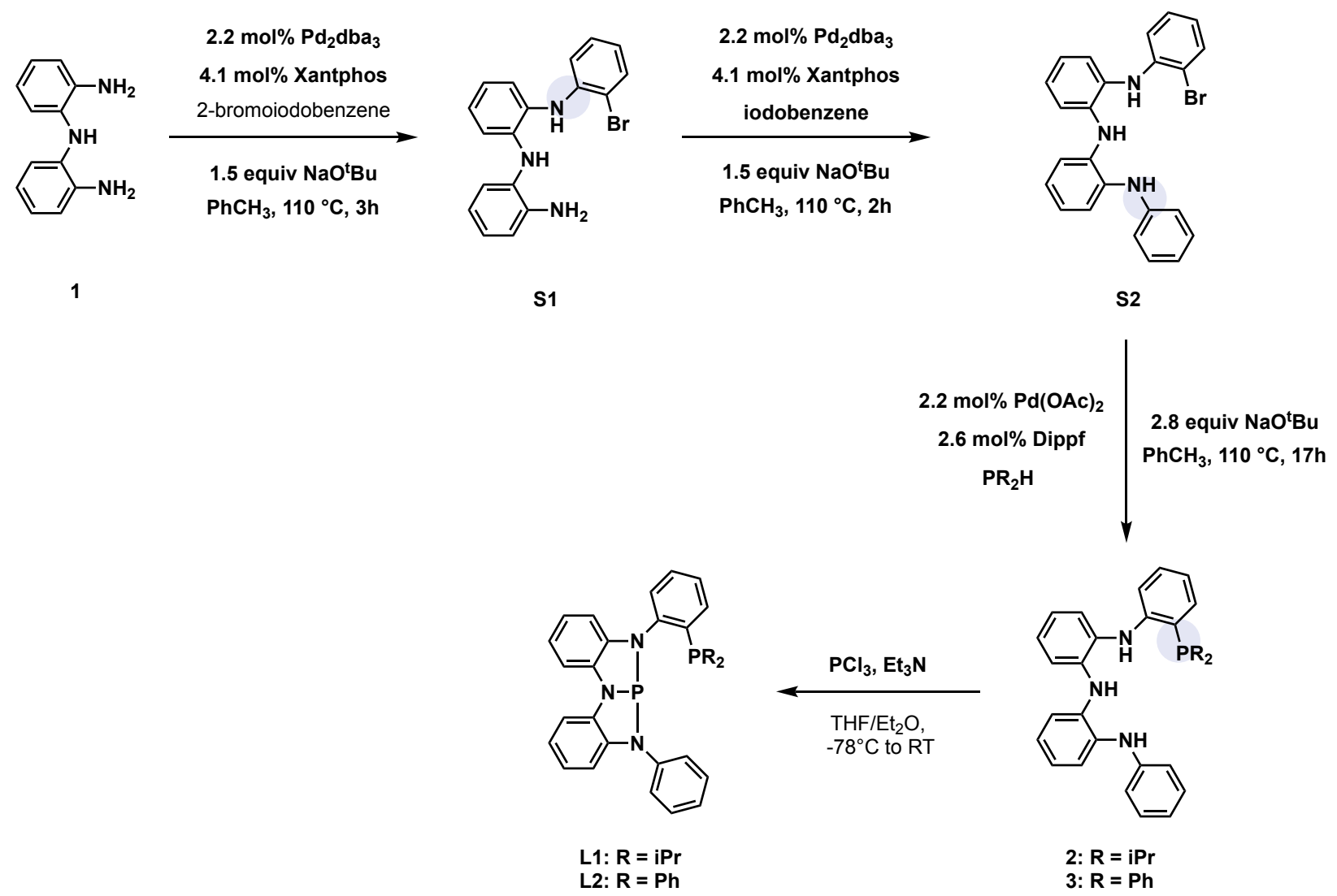

Figure S1. Synthetic Scheme for bidentate ligand L1 and L2.

N1-(2-aminophenyl)-N2-(2-bromophenyl)benzene-1,2-diamine

(S1).

$\operatorname{Bis}(2-$ aminophenyl)amine A1 (4.00g, $20.1 \mathrm{mmol}, 1.00 \mathrm{eq}), \mathrm{Pd}_{2}(\mathrm{dba})_{3}$ (405 mg, $0.44 \mathrm{mmol}, 2.20 \mathrm{~mol} \%$ ), Xantphos (477 mg, $0.82 \mathrm{mmol}, 4.1 \mathrm{~mol} \%$ ), $\mathrm{NaO}^{t} \mathrm{Bu}(2.89 \mathrm{~g}, 30.2 \mathrm{mmol}, 1.50 \mathrm{eq}$ ), and $80 \mathrm{~mL}$ of toluene were combined in a $200 \mathrm{~mL}$ Schlenk flask equipped with a stir bar. The Schlenk flask was sealed with a rubber septum and removed from the glovebox. Bromoiodobenzene (5.96 g, 21.1 mmol, $1.05 \mathrm{eq})$ was then injected using a syringe. The resulting reaction mixture was heated to $110^{\circ} \mathrm{C}$ with vigorous stirring for $3 \mathrm{~h}$. After it was cooled to room temperature, the mixture was filtered through a bed of silica, which was then washed with $\mathrm{CH}_{2} \mathrm{Cl}_{2}(40 \mathrm{~mL})$. The compound was further purified using ethyl acetate/hexanes $\left(20 \%\right.$ EtOAc). The appropriate fractions $\left(\mathrm{R}_{\mathrm{f}}=0.45\right.$ in $20 \%$ EtOAc) were collected and evaporated to dryness yielding (S1) as a yellow gel $(2.05 \mathrm{~g}, 28.0 \%$ yield). ${ }^{1} \mathrm{H}$ NMR $\left(500 \mathrm{MHz}, \mathrm{CD}_{3} \mathrm{Cl}_{-} \mathrm{d}_{1}, 23{ }^{\circ} \mathrm{C}\right) \delta(\mathrm{ppm}): 7.52(\mathrm{~d}, J=7.5 \mathrm{~Hz}, 1 \mathrm{H}), 7.21(\mathrm{~d}, J=7.6$ $\mathrm{Hz}, 1 \mathrm{H}), 7.15(\mathrm{t}, J=7.7 \mathrm{~Hz}, 1 \mathrm{H}), 7.11(\mathrm{t}, J=7.6 \mathrm{~Hz}, 1 \mathrm{H}), 7.04(\mathrm{dd}, J=7.7,12.1 \mathrm{~Hz}, 2 \mathrm{H}), 6.85(\mathrm{t}$, $J=7.6 \mathrm{~Hz}, 1 \mathrm{H}), 6.80(\mathrm{dd}, J=7.7,14.4 \mathrm{~Hz}, 2 \mathrm{H}), 6.75(\mathrm{dd}, J=4.6,8.0 \mathrm{~Hz}, 2 \mathrm{H}), 6.71(\mathrm{~d}, J=7.5$ $\mathrm{Hz}, 1 \mathrm{H}), 5.87(\mathrm{~s}, 1 \mathrm{H}), 5.62(\mathrm{br}, 1 \mathrm{H}), 4.04(\mathrm{br}, 2 \mathrm{H}) .{ }^{13} \mathrm{C}\left\{{ }^{1} \mathrm{H}\right\} \mathrm{NMR}\left(126 \mathrm{MHz}, \mathrm{CD}_{3} \mathrm{Cl}-\mathrm{d}_{1}, 23{ }^{\circ} \mathrm{C}\right) \delta$ (ppm): 143.1, 141.8, 141.6, 132.9, 128.5, 128.2, 128.0, 127.0, 126.4, 126.2, 125.6, 120.2, 119.7, 119.6, 116.5, 114.9, 114.7, 111.0. HRMS (DART) m/z calc for $\mathrm{C}_{18} \mathrm{H}_{17} \mathrm{BrN}_{3}[\mathrm{M}+\mathrm{H}]^{+}: 354.06004$, found 354.05906 . 
N1-(2-bromophenyl)-N2-(2-(phenylamino)phenyl)benzene-1,2-diamine (S2). S1 (1.90g, 5.36 mmol, 1.00 eq), $\mathrm{Pd}_{2}(\mathrm{dba})_{3}$ (110 mg, $0.12 \mathrm{mmol}, 2.20 \mathrm{~mol} \%$ ), Xantphos (128 mg, $0.22 \mathrm{mmol}, 4.1$ $\mathrm{mol} \%$ ), $\mathrm{NaO}{ }^{t} \mathrm{Bu}(774 \mathrm{mg}, 8.09 \mathrm{mmol}, 1.50 \mathrm{eq})$, and $25 \mathrm{~mL}$ of toluene were combined in a $100 \mathrm{~mL}$ Schlenk flask equipped with a stir bar. The Schlenk flask was sealed with a rubber septum and removed from the glovebox. Iodobenzene $(1.09 \mathrm{~g}, 5.36 \mathrm{mmol}, 1.00 \mathrm{eq})$ was then injected using a syringe. The resulting reaction mixture was heated to $110{ }^{\circ} \mathrm{C}$ with vigorous stirring for $3 \mathrm{~h}$. After it was cooled to room temperature, the mixture was filtered through a bed of silica, which was then washed with $\mathrm{CH}_{2} \mathrm{Cl}_{2}(40 \mathrm{~mL})$. The compound was further purified using ethyl acetate/hexanes $(2 \%$ EtOAc). The appropriate fractions $\left(\mathrm{R}_{\mathrm{f}}=0.30\right.$ in $2 \%$ EtOAc) were collected and evaporated to dryness yielding $\mathbf{S 2}$ as a yellow gel (1.41 g, 61.1\% yield). ${ }^{1} \mathrm{H}$ NMR $\left(500 \mathrm{MHz}, \mathrm{CD}_{3} \mathrm{Cl}-\mathrm{d}_{1}, 23{ }^{\circ} \mathrm{C}\right)$ $\delta$ (ppm): 7.55 (d, $J=7.9 \mathrm{~Hz}, 1 \mathrm{H}), 7.34$ (d, $J=7.5 \mathrm{~Hz}, 1 \mathrm{H}), 7.27$ (t, $J=7.4 \mathrm{~Hz}, 3 \mathrm{H}), 7.16$ (dt, $J=$ 7.4, $15.2 \mathrm{~Hz}, 3 \mathrm{H}), 7.09$ (d, $J=7.9 \mathrm{~Hz}, 1 \mathrm{H}), 7.04$ (br, 2H), 6.98 (br, 4H), 6.84 (d, $J=8.1 \mathrm{~Hz}, 1 \mathrm{H})$, $6.76(\mathrm{t}, J=7.6 \mathrm{~Hz}, 1 \mathrm{H}), 5.95(\mathrm{br}, 3 \mathrm{H}) .{ }^{13} \mathrm{C}\left\{{ }^{1} \mathrm{H}\right\} \mathrm{NMR}\left(126 \mathrm{MHz}, \mathrm{CD}_{3} \mathrm{Cl}_{-} \mathrm{d}_{1}, 23{ }^{\circ} \mathrm{C}\right) \delta(\mathrm{ppm}): 143.9$, $142.7,139.4,135.6,134.5,132.9,130.6,129.5,128.5,126.1,125.1,123.8,123.2,121.5,120.9$, 120.6, 120.3 $117.8,117.5,115.1,111.5$. HRMS (DART) $\mathrm{m} / \mathrm{z}$ calc for $\mathrm{C}_{24} \mathrm{H}_{21} \mathrm{BrN}_{3}[\mathrm{M}+\mathrm{H}]^{+}$: 430.09134 , found 430.09029 .

N1-(2-diisopropylphosphinophenyl)-N2-(2-(phenylamino)phenyl)benzene-1,2-diamine (2). A $100 \mathrm{~mL}$ round bottom flask equipped with a stir bar was charged with $\mathrm{Pd}(\mathrm{OAc})_{2}(5.3 \mathrm{mg}, 0.023$ mmol, $4.0 \mathrm{~mol} \%$ ), DiPPF (11.6 mg, $0.028 \mathrm{mmol}, 4.9 \mathrm{~mol} \%)$, and toluene (4 mL). After the mixture was stirred for $5 \mathrm{~min}, \mathrm{NaO}^{t} \mathrm{Bu}(78.0 \mathrm{mg}, 0.812 \mathrm{mmol}, 1.40 \mathrm{eq})$ was added, followed by diisopropylphosphine $(68.7 \mathrm{mg}, 0.581 \mathrm{mmol}, 1.00 \mathrm{eq})$. The round flask was sealed with a cap containing a PTFE septum and removed from the glovebox. Compound S2 (250.0 mg, 0.581 $\mathrm{mmol}, 1.00 \mathrm{eq})$ in toluene $(2 \mathrm{~mL})$ was added via syringe. The vial was then heated to $110^{\circ} \mathrm{C}$ with vigorous stirring for $21 \mathrm{~h}$, at which point complete conversion was confirmed by ${ }^{1} \mathrm{H}$ and ${ }^{31} \mathrm{P} \mathrm{NMR}$ of an aliquot of the reaction mixture. The cooled reaction mixture was then filtered through a bed of silica and washed with $\mathrm{CH}_{2} \mathrm{Cl}_{2}(20 \mathrm{~mL})$. The solution was concentrated, and the resulting crude material was purified by flash chromatography (ethyl acetate/hexanes (2\% EtOAc)), giving the product 2 as a yellow oil in $75.2 \%$ yield $(204.3 \mathrm{mg}, 0.437 \mathrm{mmol}) .{ }^{1} \mathrm{H}$ NMR $\left(500 \mathrm{MHz}, \mathrm{CD}_{3} \mathrm{Cl}-\mathrm{d}_{1}\right.$, $\left.23{ }^{\circ} \mathrm{C}\right) \delta(\mathrm{ppm}): 7.31(\mathrm{~d}, J=7.4 \mathrm{~Hz}, 2 \mathrm{H}), 7.22(\mathrm{ddd}, J=25.4,13.7,8.6 \mathrm{~Hz}, 5 \mathrm{H}), 7.04(\mathrm{t}, J=8.6$ $\mathrm{Hz}, 3 \mathrm{H}), 6.94(\mathrm{qd}, J=15.6,7.4 \mathrm{~Hz}, 6 \mathrm{H}), 6.82(\mathrm{~m}, 2 \mathrm{H}), 5.92(\mathrm{~s}, 1 \mathrm{H}), 5.63(\mathrm{~s}, 1 \mathrm{H}), 2.14(\mathrm{~m}, 2 \mathrm{H})$, $1.12(\mathrm{dd}, J=15.8,6.9 \mathrm{~Hz}, 6 \mathrm{H}), 0.93(\mathrm{dd}, J=12.0,6.9 \mathrm{~Hz}, 6 \mathrm{H}) .{ }^{13} \mathrm{C}\left\{{ }^{1} \mathrm{H}\right\} \mathrm{NMR}\left(126 \mathrm{MHz}, \mathrm{CD}_{3} \mathrm{Cl}-\right.$ $\left.\mathrm{d}_{1}, 23{ }^{\circ} \mathrm{C}\right) \delta(\mathrm{ppm}): 150.8(\mathrm{~d}, J=18.5 \mathrm{~Hz}), 143.8,139.1,135.9,134.2,133.3,131.9,130.0,129.3$, 125.1, 124.2, 123.3, 122.6, 121.2, 121.1, 120.7, 119.4, 119.3, 118.9, 117.7, 116.9, 114.0, 23.3 (d, $J=9.0 \mathrm{~Hz}), 20.2(\mathrm{~d}, J=17.9 \mathrm{~Hz}), 18.9(\mathrm{~d}, J=8.2 \mathrm{~Hz}) .{ }^{31} \mathrm{P}\left\{{ }^{1} \mathrm{H}\right\} \mathrm{NMR}\left(203 \mathrm{MHz}, \mathrm{CD}_{3} \mathrm{Cl}-\mathrm{d}_{1}\right) \delta$ (ppm): -16.0 (s). HRMS (DART) m/z calc for $\mathrm{C}_{30} \mathrm{H}_{35} \mathrm{~N}_{3} \mathrm{P}[\mathrm{M}+\mathrm{H}]^{+}$: 468.25631, found 468.25733. 
N1-(2-diphenylphosphinophenyl)-N2-(2-(phenylamino)phenyl)benzene-1,2-diamine (3). A $100 \mathrm{~mL}$ round bottom flask equipped with a stir bar was charged with $\mathrm{Pd}(\mathrm{OAc})_{2}(5.5 \mathrm{mg}, 0.024$ mmol, $4.00 \mathrm{~mol} \%$ ), DiPPF (12.1 mg, $0.029 \mathrm{mmol}, 4.78 \mathrm{~mol} \%)$, and toluene (4 mL). After the mixture was stirred for $5 \mathrm{~min}, \mathrm{NaO}^{t} \mathrm{Bu}(81 \mathrm{mg}, 0.843 \mathrm{mmol}, 1.40 \mathrm{eq})$ was added, followed by diphenylphosphine (113 mg, $0.607 \mathrm{mmol}, 1.00 \mathrm{eq}$ ). The round flask was sealed with a cap containing a PTFE septum and removed from the glovebox. Compound S2 (261.4 mg, 0.607 mmol, $1.00 \mathrm{eq})$ in toluene $(2 \mathrm{~mL})$ was added via syringe. The vial was then heated to $110^{\circ} \mathrm{C}$ with vigorous stirring for $21 \mathrm{~h}$, at which point complete conversion was confirmed by ${ }^{1} \mathrm{H}$ and ${ }^{31} \mathrm{P}$ NMR of an aliquot of the reaction mixture. The cooled reaction mixture was then filtered through a bed of silica and washed with $\mathrm{CH}_{2} \mathrm{Cl}_{2}(20 \mathrm{~mL})$. The solution was concentrated, and the resulting crude material was purified by flash chromatography (ethyl acetate/hexanes (2\% EtOAc)), giving the product 3 as a yellow oil in $68.0 \%$ yield $(221 \mathrm{mg}, 0.413 \mathrm{mmol}) .{ }^{1} \mathrm{H} \mathrm{NMR}\left(500 \mathrm{MHz}, \mathrm{CD}_{3} \mathrm{Cl}-\mathrm{d}_{1}\right.$, $\left.23{ }^{\circ} \mathrm{C}\right) \delta(\mathrm{ppm}): 7.33(\mathrm{~m}, 10 \mathrm{H}), 7.26(\mathrm{dd}, J=15.7,7.9 \mathrm{~Hz}, 4 \mathrm{H}), 7.13(\mathrm{~d}, J=7.7 \mathrm{~Hz}, 1 \mathrm{H}), 7.01(\mathrm{t}, J$ $=7.5 \mathrm{~Hz}, 2 \mathrm{H}), 6.94(\mathrm{~m}, 5 \mathrm{H}), 6.86(\mathrm{~m}, 5 \mathrm{H}), 6.13(\mathrm{~d}, J=5.8 \mathrm{~Hz}, 1 \mathrm{H}), 5.48(\mathrm{~s}, 1 \mathrm{H}), 5.40(\mathrm{~s}, 1 \mathrm{H})$. ${ }^{13} \mathrm{C}\left\{{ }^{1} \mathrm{H}\right\}$ NMR $\left(126 \mathrm{MHz}, \mathrm{CD}_{3} \mathrm{Cl}-\mathrm{d}_{1}, 23{ }^{\circ} \mathrm{C}\right) \delta(\mathrm{ppm}): 147.7,147.6,143.6,138.8,135.2,135.1$, $134.3,133.9$, 133.7, 133.6, 131.3, 130.3, 129.4, 129.1, 128.8, 128.7, 125.2, 124.2, 123.6, 123.1, $122.2,122.0,121.3,120.7,120.4,118.6,117.7,117.4,114.7 .{ }^{31} \mathrm{P}\left\{{ }^{1} \mathrm{H}\right\} \mathrm{NMR}\left(203 \mathrm{MHz}, \mathrm{CD}_{3} \mathrm{Cl}-\right.$ $\left.\mathrm{d}_{1}\right) \delta(\mathrm{ppm}):-18.5(\mathrm{~s})$. HRMS (DART) $\mathrm{m} / \mathrm{z}$ calc for $\mathrm{C}_{36} \mathrm{H}_{31} \mathrm{~N}_{3} \mathrm{P}[\mathrm{M}+\mathrm{H}]^{+}:$536.22501, found 536.22433 .

L1. Triamine phosphine 2 (400 $\mathrm{mg}, 0.855 \mathrm{mmol}, 1.00 \mathrm{eq})$ and triethylamine $(259.5 \mathrm{mg}, 2.565$ $\mathrm{mmol}, 3.00 \mathrm{eq})$ was dissolved in $\mathrm{THF} / \mathrm{Et}_{2} \mathrm{O}(1 / 2,8 \mathrm{~mL})$. To the solution was added phosphorus trichloride $(0.075 \mathrm{~mL}, 0.855 \mathrm{mmol}, 1.00 \mathrm{eq})$ dropwise via syringe. The reaction mixture was stirred at $-78^{\circ} \mathrm{C}$ for $1 \mathrm{~h}$ and then warmed to room temperature. After $4 \mathrm{~h}$ of stirring at room temperature, the solvent was removed, and the resulting solid mixture was brought into a nitrogen-filled glovebox. The resulting thick white solid mixture was re-dissolved in $\mathrm{Et}_{2} \mathrm{O}(20 \mathrm{~mL})$ and the suspension was pipette filtered through glass paper to remove triethylammonium salt. The filtrate was concentrated in vacuo to afford pure product of $\mathbf{L 1}$ as off-white solids (288 $\mathrm{mg}, 0.581 \mathrm{mmol}$, 68.0\%). ${ }^{1} \mathrm{H}$ NMR $\left(500 \mathrm{MHz}, \mathrm{C}_{6} \mathrm{D}_{6}-\mathrm{d}_{6}, 23{ }^{\circ} \mathrm{C}\right) \delta(\mathrm{ppm}): 7.41(\mathrm{dd}, J=15.4,7.4 \mathrm{~Hz}, 2 \mathrm{H}), 7.33(\mathrm{~d}, J$ $=6.9 \mathrm{~Hz}, 1 \mathrm{H}), 7.07(\mathrm{~m}, 5 \mathrm{H}), 6.93(\mathrm{t}, J=7.2 \mathrm{~Hz}, 2 \mathrm{H}), 6.81(\mathrm{~m}, 3 \mathrm{H}), 6.72(\mathrm{~m}, 3 \mathrm{H}), 6.19(\mathrm{~d}, J=7.0$ $\mathrm{Hz}, 1 \mathrm{H}), 1.77(\mathrm{~m}, 1 \mathrm{H}), 1.60(\mathrm{~m}, 1 \mathrm{H}), 0.93(\mathrm{dd}, J=13.6,6.4 \mathrm{~Hz}, 3 \mathrm{H}), 0.77(\mathrm{~m}, 6 \mathrm{H}), 0.64(\mathrm{dd}, J=$ 11.5, 7.0 Hz, 3H). ${ }^{13} \mathrm{C}\left\{{ }^{1} \mathrm{H}\right\}$ NMR $\left(126 \mathrm{MHz}, \mathrm{C}_{6} \mathrm{D}_{6}-\mathrm{d}_{6}, 23{ }^{\circ} \mathrm{C}\right) \delta(\mathrm{ppm}): 145.6(\mathrm{dd}, J=24.4,11.3$ $\mathrm{Hz}), 142.0,141.9,141.6(\mathrm{~d}, J=8.0 \mathrm{~Hz}), 141.1,141.0,138.8,138.6,138.1,134.4(\mathrm{~d}, J=2.2 \mathrm{~Hz})$, $131.5,130.3,129.3,127.4,125.2,125.0(\mathrm{~d}, J=5.7 \mathrm{~Hz}), 124.3,124.0,120.3,119.8,118.5(\mathrm{~d}, J=$ $5.2 \mathrm{~Hz}), 117.9$ (d, $J=5.3 \mathrm{~Hz}), 111.5,111.3,25.2(\mathrm{~d}, J=14.1 \mathrm{~Hz}), 21.0(\mathrm{~d}, J=8.0 \mathrm{~Hz}), 20.0(\mathrm{dd}$, $J=38.3,16.0 \mathrm{~Hz}) .{ }^{31} \mathrm{P}\left\{{ }^{1} \mathrm{H}\right\} \mathrm{NMR}\left(203 \mathrm{MHz}, \mathrm{C}_{6} \mathrm{D}_{6}-\mathrm{d}_{6}\right) \delta(\mathrm{ppm}): 154.3(\mathrm{~d}, J=86.0 \mathrm{~Hz}),-7.93(\mathrm{~d}$, $\mathrm{J}=86.0 \mathrm{~Hz}$ ). HRMS (DART) $\mathrm{m} / \mathrm{z}$ calc for $\mathrm{C}_{30} \mathrm{H}_{32} \mathrm{~N}_{3} \mathrm{P}_{2}[\mathrm{M}+\mathrm{H}]^{+}:$496.20660, found 496.20624. 
L2. Triamine phosphine 3 (133 mg, $0.248 \mathrm{mmol}, 1.00 \mathrm{eq})$ and triethylamine $(75.3 \mathrm{mg}, 0.744 \mathrm{mmol}$, $3.00 \mathrm{eq})$ was dissolved in $\mathrm{THF} / \mathrm{Et}_{2} \mathrm{O}(1 / 2,8 \mathrm{~mL})$. To the solution was added phosphorus trichloride $(0.022 \mathrm{~mL}, 0.249 \mathrm{mmol}, 1.00 \mathrm{eq})$ dropwise via syringe. The reaction mixture was stirred at $-78^{\circ} \mathrm{C}$ for $1 \mathrm{~h}$ and then warmed to room temperature. After $4 \mathrm{~h}$ of stirring at room temperature, the solvent was removed, and the resulting solid mixture was brought into a nitrogen-filled glovebox. The resulting thick white solid mixture was re-dissolved in $\mathrm{Et}_{2} \mathrm{O}(20 \mathrm{~mL})$ and the suspension was pipette filtered through glass paper to remove triethylammonium salt. The filtrate was concentrated in vacuo to afford pure product of $\mathbf{L 2}$ as off-white solids (116 mg, $0.206 \mathrm{mmol}, 83.0 \%$ ). Crystals suitable for single-crystal diffraction analysis were obtained from a slow diffusion of pentane into an $\mathrm{Et}_{2} \mathrm{O}$ solution at $-35{ }^{\circ} \mathrm{C} .{ }^{1} \mathrm{H}$ NMR $\left(500 \mathrm{MHz}, \mathrm{C}_{6} \mathrm{D}_{6}-\mathrm{d}_{6}, 23{ }^{\circ} \mathrm{C}\right) \delta(\mathrm{ppm}): 7.43(\mathrm{~d}, J=6.7 \mathrm{~Hz}$, $1 \mathrm{H}), 7.38$ (d, $J=7.6 \mathrm{~Hz}, 1 \mathrm{H}), 7.33$ (d, $J=7.9 \mathrm{~Hz}, 1 \mathrm{H}), 7.26(\mathrm{br}, 2 \mathrm{H}), 7.22(\mathrm{~d}, J=7.8 \mathrm{~Hz}, 2 \mathrm{H})$, $7.09(\mathrm{~m}, 1 \mathrm{H}), 7.02(\mathrm{dt}, J=15.5,7.4 \mathrm{~Hz}, 5 \mathrm{H}), 6.94(\mathrm{br}, 5 \mathrm{H}), 6.88(\mathrm{~m}, 6 \mathrm{H}), 6.65(\mathrm{t}, J=7.6 \mathrm{~Hz}, 1 \mathrm{H})$, $6.57(\mathrm{t}, J=7.6 \mathrm{~Hz}, 1 \mathrm{H}), 5.87(\mathrm{~d}, J=7.7 \mathrm{~Hz}, 1 \mathrm{H}) .{ }^{13} \mathrm{C}$ NMR $(101 \mathrm{MHz}$, Chloroform- $d$ ) $\delta 143.8$ (dd, $J=24.2,11.7 \mathrm{~Hz}), 142.6(\mathrm{~d}, J=8.4 \mathrm{~Hz}), 141.1(\mathrm{dd}, J=5.9,2.3 \mathrm{~Hz}), 140.6(\mathrm{~d}, J=14.0 \mathrm{~Hz}), 140.2$ $(\mathrm{d}, J=18.2 \mathrm{~Hz}), 139.5(\mathrm{~d}, J=5.9 \mathrm{~Hz}), 137.4,135.5,134.3(\mathrm{~d}, J=22.0 \mathrm{~Hz}), 133.4(\mathrm{~d}, J=19.0 \mathrm{~Hz})$, $130.7,129.3,128.5,128.3(\mathrm{~d}, J=5.9 \mathrm{~Hz}), 128.1,128.0,125.4,125.0$ (d, $J=6.8 \mathrm{~Hz}), 124.7,123.6$, 120.6, 119.1, $118.8(\mathrm{~d}, J=6.1 \mathrm{~Hz}), 116.8(\mathrm{~d}, J=4.6 \mathrm{~Hz}), 111.89,110.72 .{ }^{31} \mathrm{P}\left\{{ }^{1} \mathrm{H}\right\} \mathrm{NMR}(203$ $\left.\mathrm{MHz}, \mathrm{C}_{6} \mathrm{D}_{6}-\mathrm{d}_{6}\right) \delta(\mathrm{ppm}): 155.6(\mathrm{~d}, J=48 \mathrm{~Hz}),-16.4(\mathrm{~d}, \mathrm{~J}=47 \mathrm{~Hz})$. HRMS (DART) $\mathrm{m} / \mathrm{z}$ calc for $\mathrm{C}_{36} \mathrm{H}_{28} \mathrm{~N}_{3} \mathrm{P}_{2}[\mathrm{M}+\mathrm{H}]^{+}:$564.17530, found 564.17471.

PtCIMeiPr 2 PNNNP (4). A solution of $\mathbf{L 1}(20 \mathrm{mg}, 0.04 \mathrm{mmol}, 1.00 \mathrm{eq})$ in $2 \mathrm{~mL}$ of $\mathrm{C}_{6} \mathrm{H}_{6}$ was added to a solution of $(\mathrm{cod}) \mathrm{Pt}(\mathrm{Me})(\mathrm{Cl})(14.4 \mathrm{mg}, 0.04 \mathrm{mmol}, 1.00 \mathrm{eq})$ in $2 \mathrm{~mL}$ of $\mathrm{C}_{6} \mathrm{H}_{6}$ to prompt an immediate color change to light yellow/green. After stirring at ambient temperature for $0.5 \mathrm{hr}$, the reaction mixture was concentrated to dryness and the residue was taken up in $\mathrm{Et}_{2} \mathrm{O}$ and solvent was decanted, and the residue was dried in vacuo to afford $27.2 \mathrm{mg}$ of the title compound as an off-white solid (90.0\% yield). The solid was re-dissolved in $\mathrm{CHCl}_{3}$ and the resulting light yellow solution was filtered through a tightly packed glass paper pipette filter and placed in a pentane diffusion chamber to grow colorless block shaped crystals. If upon complete diffusion of pentane into the solution the crystal yield is low, the vial was placed in a freezer at $-35^{\circ} \mathrm{C}$ for $24 \mathrm{~h}$ prior to crystal collection. The supernatant was removed and the crystals washed with additional pentane. The crystals were then dried under vacuum to yield a mixture of 4-cis and 4-trans as a white crystalline material. Crystals suitable for single-crystal diffraction analysis were obtained from a slow diffusion of pentane into an $\mathrm{CHCl}_{3}$ solution at $25{ }^{\circ} \mathrm{C}$. ${ }^{1} \mathrm{H} \mathrm{NMR}\left(500 \mathrm{MHz}, \mathrm{CDCl}_{3}-\mathrm{d}_{1}\right)$ $\delta 7.66(\mathrm{tt}, J=17.7,8.0 \mathrm{~Hz}, 5 \mathrm{H}), 7.57$ (d, $J=7.4 \mathrm{~Hz}, 1 \mathrm{H}), 7.54-7.35(\mathrm{~m}, 3 \mathrm{H}), 7.13$ (d, $J=7.2 \mathrm{~Hz}$, $1 \mathrm{H}), 6.95(\mathrm{q}, J=7.1 \mathrm{~Hz}, 5 \mathrm{H}), 6.81(\mathrm{~d}, J=7.5 \mathrm{~Hz}, 0 \mathrm{H}), 6.77(\mathrm{~d}, J=7.6 \mathrm{~Hz}, 1 \mathrm{H}), 6.49-6.44(\mathrm{~m}$, $0 \mathrm{H}), 6.42(\mathrm{~d}, J=7.3 \mathrm{~Hz}, 1 \mathrm{H}), 3.09$ (dt, $J=13.6,6.9 \mathrm{~Hz}, 1 \mathrm{H}), 2.87$ (d, $J=39.9 \mathrm{~Hz}, 0 \mathrm{H}), 2.70$ (dd, $1 \mathrm{H}), 2.59(\mathrm{dt}, J=13.3,6.7 \mathrm{~Hz}, 0 \mathrm{H}), 1.37(\mathrm{dd}, J=15.4,7.0 \mathrm{~Hz}, 3 \mathrm{H}), 1.16(\mathrm{dd}, J=16.0,6.9 \mathrm{~Hz}$, $1 \mathrm{H}), 0.58(\mathrm{dd}, J=16.9,7.0 \mathrm{~Hz}, 4 \mathrm{H}), 0.53(\mathrm{~d}, J=7.1 \mathrm{~Hz}, 1 \mathrm{H}) .{ }^{31} \mathrm{P}\left\{{ }^{1} \mathrm{H}\right\} \mathrm{NMR}\left(203 \mathrm{MHz}, \mathrm{CDCl}_{3}-\right.$ $\left.\mathrm{d}_{1}\right) \delta(\mathrm{ppm}): 4-c i s: 100.2\left(\mathrm{td},{ }^{1} J_{\mathrm{Pt}-\mathrm{P}}=6700,{ }^{2} J_{\mathrm{P}-\mathrm{P}}=28.0 \mathrm{~Hz}\right), 17.4\left(\mathrm{td},{ }^{1} J_{\mathrm{Pt}-\mathrm{P}}=1695,{ }^{2} J_{\mathrm{P}-\mathrm{P}}=28.0\right.$ Hz). 4-trans: $142.3\left(\mathrm{td},{ }^{1} J_{\mathrm{Pt}-\mathrm{P}}=2601,{ }^{2} J_{\mathrm{P}-\mathrm{P}}=27.0 \mathrm{~Hz}\right), 15.9\left(\mathrm{td},{ }^{1} J_{\mathrm{Pt}-\mathrm{P}}=4048,{ }^{2} J_{\mathrm{P}-\mathrm{P}}=27.0 \mathrm{~Hz}\right)$. 
${ }^{13} \mathrm{C}$ NMR (126 MHz, $\left.\mathrm{CDCl}_{3}-\mathrm{d}_{1}\right) \delta 143.15,142.80(\mathrm{~d}, J=8.5 \mathrm{~Hz}), 140.14(\mathrm{~d}, J=6.7 \mathrm{~Hz}), 139.79$, 139.69 (d, $J=11.4 \mathrm{~Hz}), 139.25,138.33,137.16(\mathrm{~d}, J=10.7 \mathrm{~Hz}), 136.77(\mathrm{~d}, J=13.1 \mathrm{~Hz}), 136.56$ (d, $J=8.4 \mathrm{~Hz}), 136.03$ (d, $J=4.0 \mathrm{~Hz}), 135.94,133.21,132.69,132.56,132.13,130.12,129.91$, 129.81, 129.60, 128.23, 128.17, 127.75, 127.68, 126.08 (d, $J=5.0 \mathrm{~Hz}), 125.31,124.58,124.18$, 124.12, 121.29 (d, $J=11.4 \mathrm{~Hz}), 121.08(\mathrm{~d}, J=5.5 \mathrm{~Hz}), 120.18(\mathrm{~d}, J=33.4 \mathrm{~Hz}), 119.46(\mathrm{~d}, J=$ $10.2 \mathrm{~Hz}), 119.22(\mathrm{~d}, J=8.9 \mathrm{~Hz}), 116.62(\mathrm{~d}, J=8.9 \mathrm{~Hz}), 115.82(\mathrm{~d}, J=10.0 \mathrm{~Hz}), 110.37,110.28$ (d, $J=5.5 \mathrm{~Hz}), 109.17$ (d, $J=5.7 \mathrm{~Hz}), 108.52,25.69$ (d, $J=38.6 \mathrm{~Hz}), 24.89,24.67,24.71-24.37$ (m), 19.23 (d, $J=4.4 \mathrm{~Hz}), 19.00$ (d, $J=94.1 \mathrm{~Hz}), 18.28-18.12(\mathrm{~m}), 18.10,16.83(\mathrm{~d}, J=5.6 \mathrm{~Hz})$, $16.53(\mathrm{~d}, J=5.2 \mathrm{~Hz}), 5.96$ (dd, $J=149.7,6.9 \mathrm{~Hz}), 3.76(\mathrm{dd}, J=85.8,5.0 \mathrm{~Hz})$. HRMS (DART) $\mathrm{m} / \mathrm{z}$ calc for $\mathrm{C}_{31} \mathrm{H}_{34} \mathrm{~N}_{3} \mathrm{P}_{2} \mathrm{Pt}[\mathrm{M}-\mathrm{Cl}]^{+}:$705.18702, found 705.18683 .

PdCliPr $_{2}$ PNNNPMe (5') To a $20 \mathrm{~mL}$ scintillation vial was added $\mathbf{L 1}(30 \mathrm{mg}, 0.060 \mathrm{mmol}, 1.00$ eq), (cod) $\mathrm{Pd}(\mathrm{Me})(\mathrm{Cl})(16.2 \mathrm{mg}, 0.060 \mathrm{mmol}, 1.00 \mathrm{eq})$, and $\mathrm{C}_{6} \mathrm{H}_{6}(4.00 \mathrm{~g})$. The reaction immediately turned dark violet. The reaction was stirred using a small Teflon coated magnetic stir bar at ambient temperature for $1 \mathrm{~h}$, during which time a light orange solution was observed. The mixture was concentrated to dryness and the residue was washed with pentane twice and dried in vacuo to afford the title complex as a yellow solid ( $40 \mathrm{mg}$, 99\% yield). The solid was re-dissolved in $\mathrm{PhCF}_{3}$ and the resulting yellow solution was filtered through a tightly packed glass paper pipette filter and placed in a pentane diffusion chamber to grow yellow needle shaped crystals. The supernatant was removed and the crystals washed with additional pentane. If upon complete diffusion of pentane into the solution the crystal yield is low, the vial was placed in a freezer at $-35^{\circ} \mathrm{C}$ for 24 $\mathrm{h}$ prior to crystal collection. The crystals were then dried under vacuum to yield $\mathbf{5}$ as a yellow crystalline material. ${ }^{1} \mathrm{H}$ NMR $\left(500 \mathrm{MHz}, \mathrm{CD}_{2} \mathrm{Cl}_{2}-\mathrm{d}_{2}, 23{ }^{\circ} \mathrm{C}\right) \delta(\mathrm{ppm}): 7.90(\mathrm{br}, 1 \mathrm{H}), 7.74(\mathrm{br}, 1 \mathrm{H})$, 7.64 (br, 2H), 7.57 (br, 1H), 7.52 (br, 2H), 7.28 (br, 3H), 7.18 (br, 1H), 7.12 (br, 1H), 7.05 (br, 2H), 6.89 (br, 2H), $6.78(\mathrm{br}, 1 \mathrm{H}), 2.81(\mathrm{~m}, 1 \mathrm{H}), 2.20(\mathrm{~m}, 1 \mathrm{H}), 1.60(\mathrm{~d}, J=10.7 \mathrm{~Hz}, 3 \mathrm{H}), 1.47(\mathrm{~m}$, $6 \mathrm{H}), 0.92(\mathrm{~d}, J=15.9 \mathrm{~Hz}, 6 \mathrm{H}) .{ }^{13} \mathrm{C}\left\{{ }^{1} \mathrm{H}\right\} \mathrm{NMR}\left(126 \mathrm{MHz}, \mathrm{CD}_{2} \mathrm{Cl}_{2}-\mathrm{d}_{2}, 23{ }^{\circ} \mathrm{C}\right) \delta(\mathrm{ppm}): 147.6,144.7$ (t, $J=7,5 \mathrm{~Hz}), 138.2,135.0$ (d, $J=2.8 \mathrm{~Hz}), 132.4,132.1,131.9,129.8$ (d, $J=7.5 \mathrm{~Hz}), 129.2,128.2$ (d, $J=4.0 \mathrm{~Hz}), 126.5,125.1,123.9,122.4,122.3$ (d, $J=5.3 \mathrm{~Hz}), 121.2,118.4,114.7$ (d, $J=7.8$ $\mathrm{Hz}), 111.8(\mathrm{~d}, J=7.0 \mathrm{~Hz}), 106.6,26.1(\mathrm{~d}, J=21.9 \mathrm{~Hz}), 22.7$ (d, $J=24.7 \mathrm{~Hz}), 19.8,19.4$ (d, $J=$ $5.4 \mathrm{~Hz}), 16.8(\mathrm{dd}, J=19.2,4.5 \mathrm{~Hz}), 16.2(\mathrm{~d}, J=7.8 \mathrm{~Hz}), 15.5(\mathrm{~d}, J=8.2 \mathrm{~Hz}) .{ }^{31} \mathrm{P}\left\{{ }^{1} \mathrm{H}\right\} \mathrm{NMR}(203$ $\left.\mathrm{MHz}, \mathrm{CD}_{2} \mathrm{Cl}_{2}-\mathrm{d}_{2}\right) \delta(\mathrm{ppm}): 31.0\left(\mathrm{~d},{ }^{2} J_{\mathrm{P}-\mathrm{P}}=42.5 \mathrm{~Hz}\right), 0.50\left(\mathrm{~d},{ }^{2} J_{\mathrm{P}-\mathrm{P}}=42.2 \mathrm{~Hz}\right) .{ }^{31} \mathrm{P}$ NMR $(203$ $\left.\mathrm{MHz}, \mathrm{CD}_{2} \mathrm{Cl}_{2}-\mathrm{d}_{2}\right) \delta(\mathrm{ppm}): 30.9(\mathrm{~m}), 0.50\left(\mathrm{dq},{ }^{2} \mathrm{~J}_{\mathrm{P}-\mathrm{P}}=42.2,{ }^{1} J_{\mathrm{P}-\mathrm{C}}=10.5 \mathrm{~Hz}\right)$. Crystals suitable for single-crystal diffraction analysis were obtained from $\mathrm{PhCF}_{3}$ solution layered with pentane at -35 ${ }^{\circ} \mathrm{C}$. HRMS (DART) $\mathrm{m} / \mathrm{z}$ calc for $\mathrm{C}_{31} \mathrm{H}_{35} \mathrm{ClN}_{3} \mathrm{P}_{2} \mathrm{Pd}[\mathrm{M}+\mathrm{H}]^{+}: 652.10210$ found 652.10254 .

\section{NiCliPr 2 PNNNPMe (6')}

To a $20 \mathrm{~mL}$ scintillation vial was added $\mathbf{L 1}(20 \mathrm{mg}, 0.040 \mathrm{mmol}, 1.00 \mathrm{eq}),\left(\mathrm{Me}_{3} \mathrm{P}\right)_{2} \mathrm{Ni}(\mathrm{Me})(\mathrm{Cl})$ (11.6 mg, $0.040 \mathrm{mmol}, 1.00 \mathrm{eq}$ ), and $\mathrm{C}_{6} \mathrm{H}_{6}(4 \mathrm{~mL})$. The reaction immediately turned dark brown. The reaction was stirred using a small Teflon coated magnetic stir bar at ambient temperature for $1 \mathrm{~h}$, during which time a light orange solution was observed. The mixture was concentrated to 
dryness and the residue was washed with pentane twice and dried in vacuo to afford the title complex as a yellow solid $(8.8 \mathrm{mg}, 37 \%$ yield). The solid was re-dissolved in toluene and the resulting yellow solution was filtered through a tightly packed glass paper pipette filter and placed in a pentane diffusion chamber to grow yellow needle shaped crystals. The supernatant was removed and the crystals washed with additional pentane. If upon complete diffusion of pentane into the solution the crystal yield is low, the vial was placed in a freezer at $-35^{\circ} \mathrm{C}$ for $24 \mathrm{~h}$ prior to crystal collection. The crystals were then dried under vacuum to yield $\mathbf{6}$ as an orange crystalline material. Crystals suitable for single-crystal diffraction analysis were obtained from toluene solution layered with pentane at $-35{ }^{\circ} \mathrm{C} .{ }^{1} \mathrm{H}$ NMR $\left(500 \mathrm{MHz}, \mathrm{C}_{6} \mathrm{D}_{6}\right) \delta 7.84(\mathrm{~d}, J=8.1 \mathrm{~Hz}, 1 \mathrm{H})$, $7.57(\mathrm{dd}, J=5.9,2.9 \mathrm{~Hz}, 1 \mathrm{H}), 7.52(\mathrm{~d}, J=7.9 \mathrm{~Hz}, 1 \mathrm{H}), 7.10(\mathrm{~m}, 4 \mathrm{H}), 7.01(\mathrm{~m}, 4 \mathrm{H}), 6.87(\mathrm{~m}, 2 \mathrm{H})$, $6.76(\mathrm{~m}, 4 \mathrm{H}), 2.11(\mathrm{sepd}, J=7.0,4.0 \mathrm{~Hz}, 1 \mathrm{H}), 2.01(\mathrm{sepd}, J=7.1,4.1 \mathrm{~Hz}, 1 \mathrm{H}), 1.56(\mathrm{td}, J=18.1$, $16.9,6.9 \mathrm{~Hz}, 6 \mathrm{H}), 1.11(\mathrm{~d}, J=6.8 \mathrm{~Hz}, 3 \mathrm{H}), 0.96(\mathrm{dd}, J=17.1,7.2 \mathrm{~Hz}, 1 \mathrm{H}), 0.47$ (dd, $J=13.5,7.0$ $\mathrm{Hz}, 3 \mathrm{H}) .{ }^{13} \mathrm{C}$ NMR $\left(101 \mathrm{MHz}, \mathrm{C}_{6} \mathrm{D}_{6}\right) \delta 148.77(\mathrm{~d}, J=6.6 \mathrm{~Hz}), 145.60,134.84,132.45,131.42$, 129.67, 129.34, 129.18, $128.16(\mathrm{~d}, J=2.4 \mathrm{~Hz}), 127.94,126.49$ (d, $J=4.5 \mathrm{~Hz}), 125.70,124.46$, $124.11(\mathrm{~d}, J=5.0 \mathrm{~Hz}), 123.24,122.96,122.27,120.93,119.32,115.51(\mathrm{~d}, J=7.1 \mathrm{~Hz}), 111.22(\mathrm{~d}$, $J=6.9 \mathrm{~Hz}), 106.56,25.23(\mathrm{~d}, J=20.8 \mathrm{~Hz}), 22.31(\mathrm{~d}, J=23.5 \mathrm{~Hz}), 20.05(\mathrm{~d}, J=3.3 \mathrm{~Hz}), 19.50(\mathrm{~d}$, $J=5.0 \mathrm{~Hz}), 17.70$ (d, $J=3.4 \mathrm{~Hz}), 16.70(\mathrm{~d}, J=4.9 \mathrm{~Hz}), 14.28 .{ }^{31} \mathrm{P} \mathrm{NMR}\left(162 \mathrm{MHz}, \mathrm{CDCl}_{3}-\mathrm{d}_{1}\right) \delta$ $30.7(\mathrm{~d}, J=86 \mathrm{~Hz}),-14.9(\mathrm{~d}, J=87 \mathrm{~Hz})$. HRMS (DART) $\mathrm{m} / \mathrm{z}$ calc for $\mathrm{C}_{31} \mathrm{H}_{35} \mathrm{ClN}_{3} \mathrm{P}_{2} \mathrm{Ni}[\mathrm{M}+\mathrm{H}]^{+}$: 604.13427, found 604.13371. 


\section{B. NMR data}

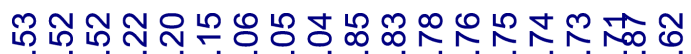

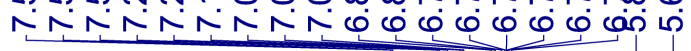

$\underset{\dot{i}}{\dot{\dagger}}$
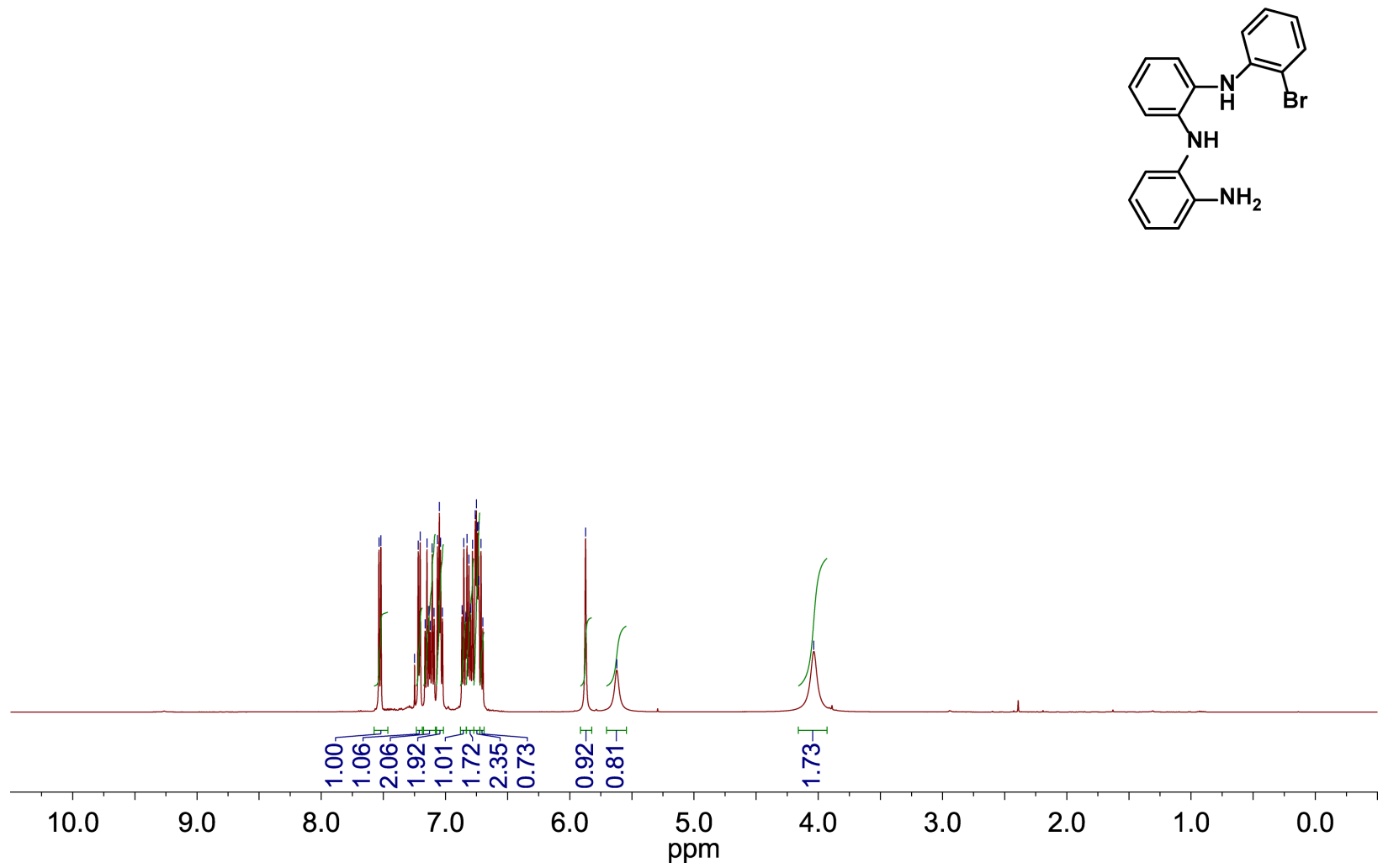

Figure S2. ${ }^{1} \mathrm{H}$ NMR spectrum of N1-(2-aminophenyl)-N2-(2-bromophenyl)benzene-1,2-diamine (S1) recorded in $\mathrm{CDCl}_{3}-\mathrm{d}_{1}$ at $23{ }^{\circ} \mathrm{C}$. 


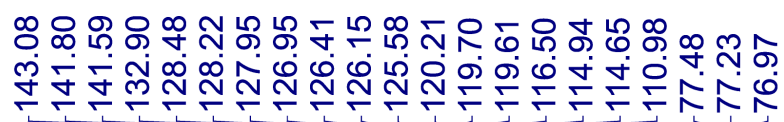
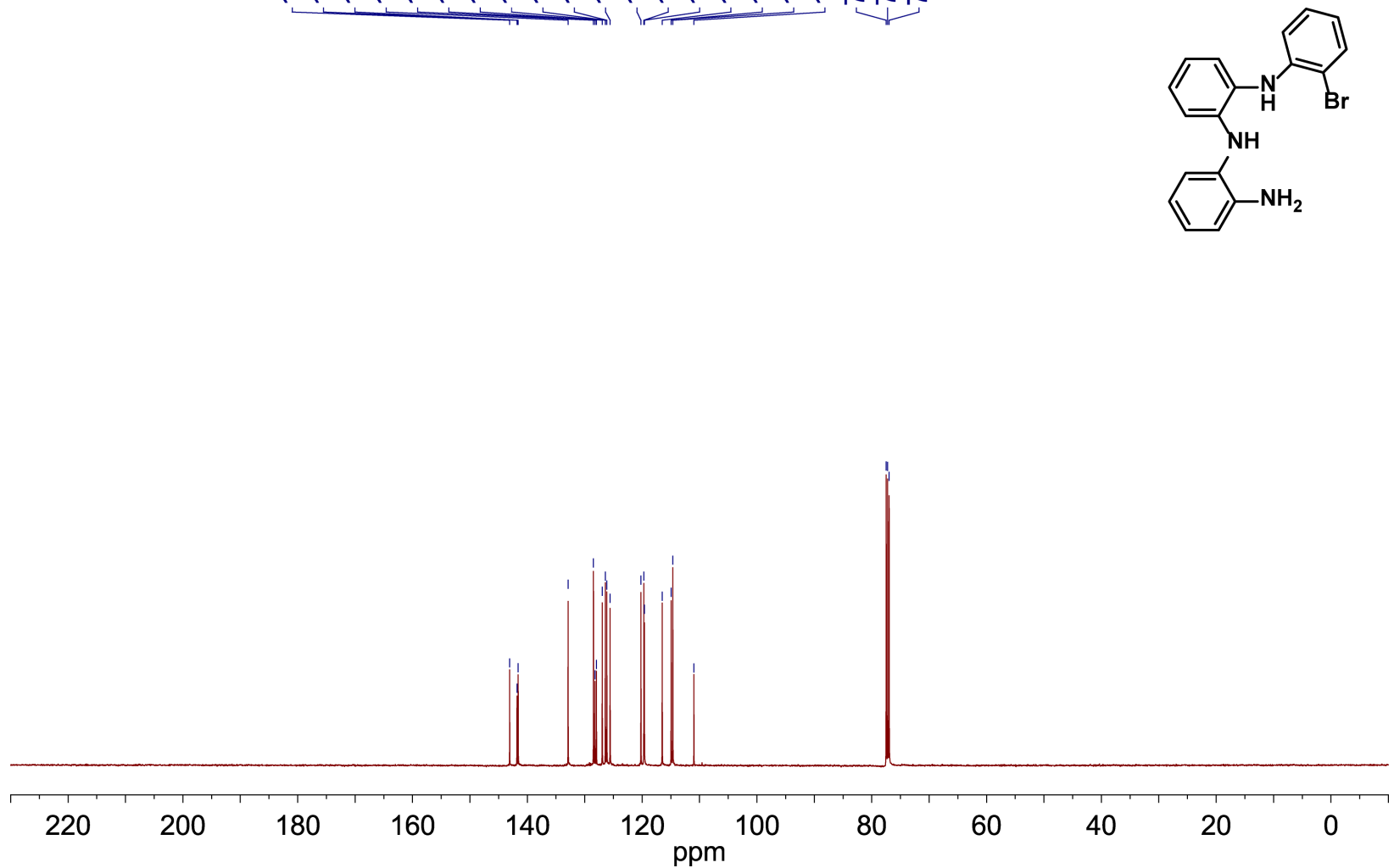

Figure S3. ${ }^{13} \mathrm{C}$ NMR spectrum of N1-(2-aminophenyl)-N2-(2-bromophenyl)benzene-1,2-diamine (S1) recorded in $\mathrm{CDCl}_{3}-\mathrm{d}_{1}$ at $23{ }^{\circ} \mathrm{C}$. 


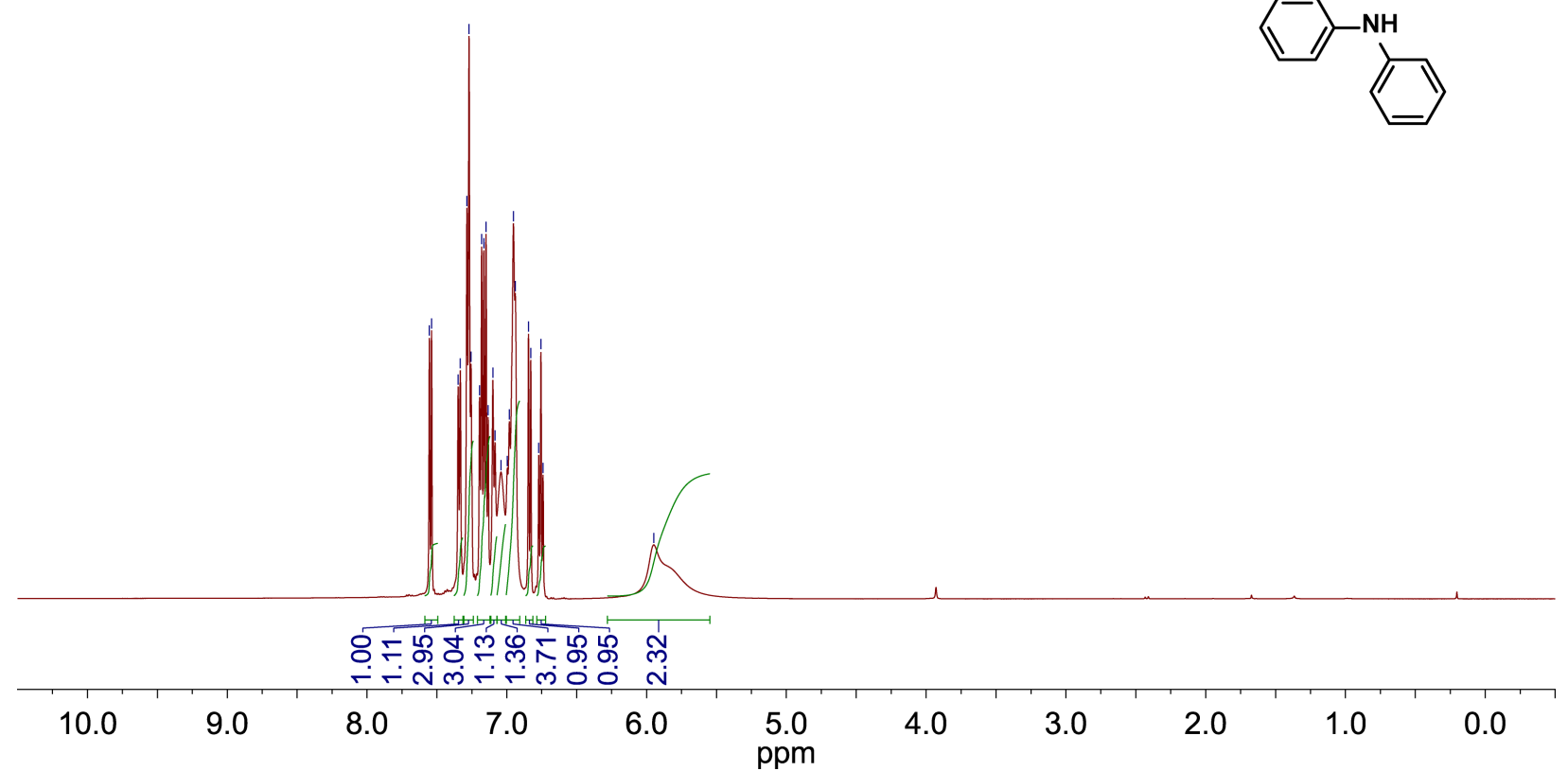

Figure S4. ${ }^{1} \mathrm{H}$ NMR spectrum of N1-(2-bromophenyl)-N2-(2-(phenylamino)phenyl)benzene-1,2diamine (S2) recorded in $\mathrm{CDCl}_{3}-\mathrm{d}_{1}$ at $23{ }^{\circ} \mathrm{C}$. 


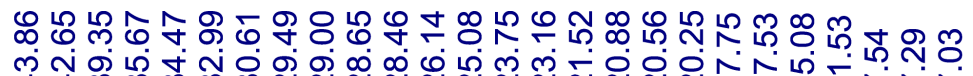

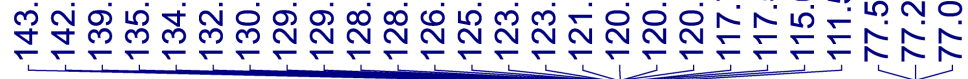
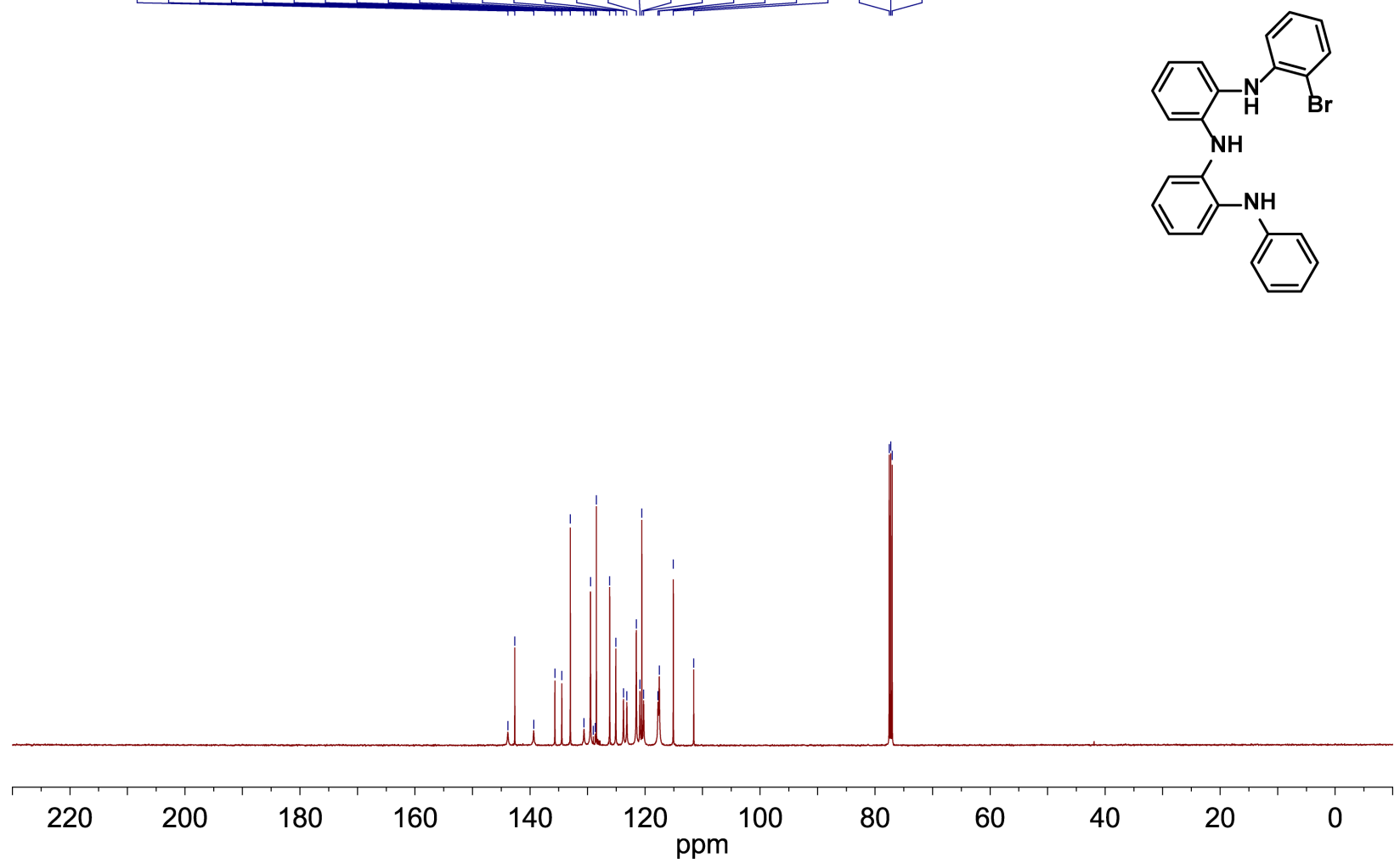

Figure S5. ${ }^{13} \mathrm{C}$ NMR spectrum of N1-(2-bromophenyl)-N2-(2-(phenylamino)phenyl)benzene-1,2diamine (S2) recorded in $\mathrm{CDCl}_{3}-\mathrm{d}_{1}$ at $23^{\circ} \mathrm{C}$. 

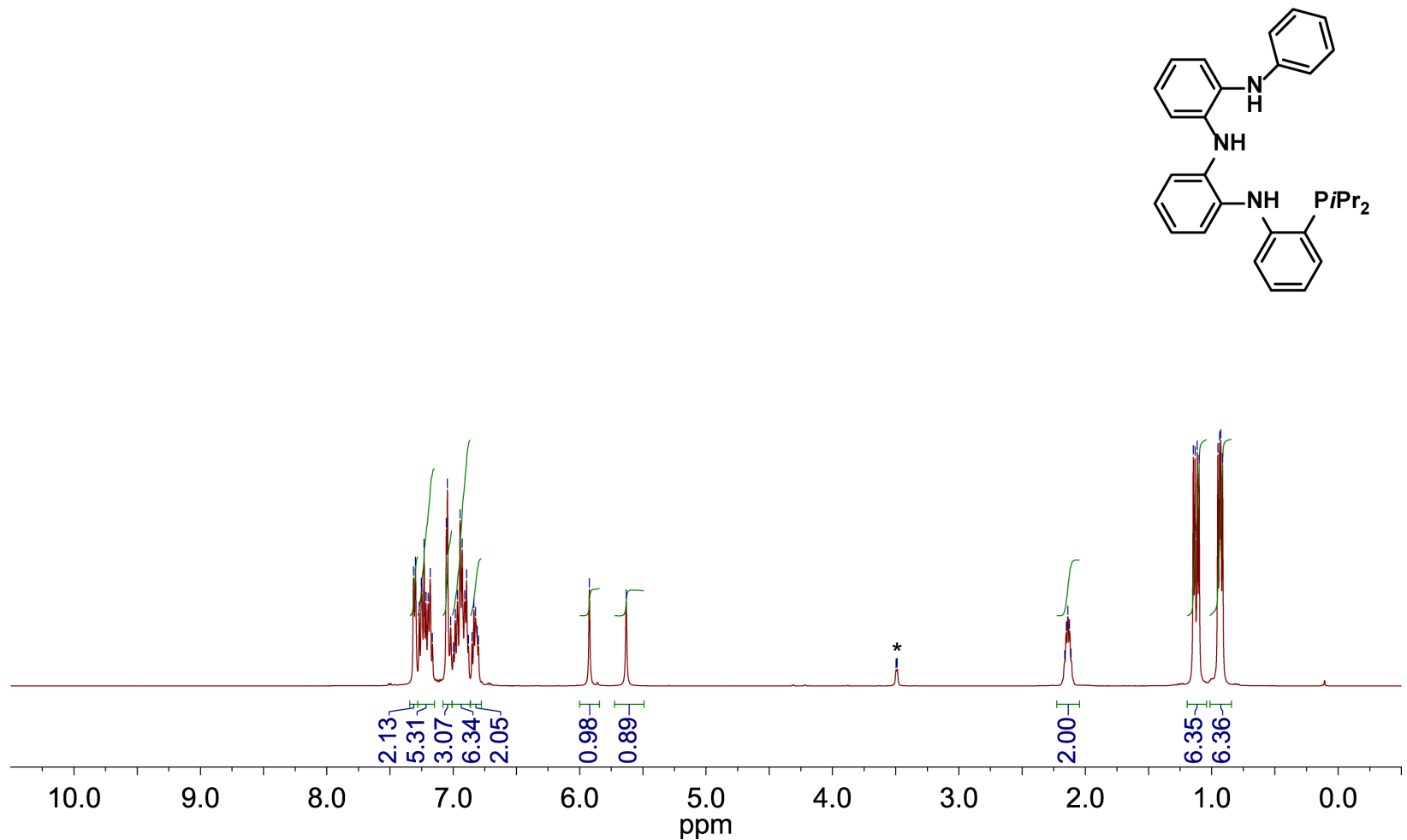

Figure S6. $\quad{ }^{1} \mathrm{H} \quad$ NMR $\quad$ spectrum of N1-(2-diisopropylphosphinophenyl)-N2-(2(phenylamino)phenyl)benzene-1,2-diamine (2) recorded in $\mathrm{CDCl}_{3}-\mathrm{d}_{1}$ at $23{ }^{\circ} \mathrm{C}$. Asterisk symbol (*) denotes residual ${ }^{1} \mathrm{H}$ peak of small amounts of $\mathrm{MeOH}$ from crystallization. 

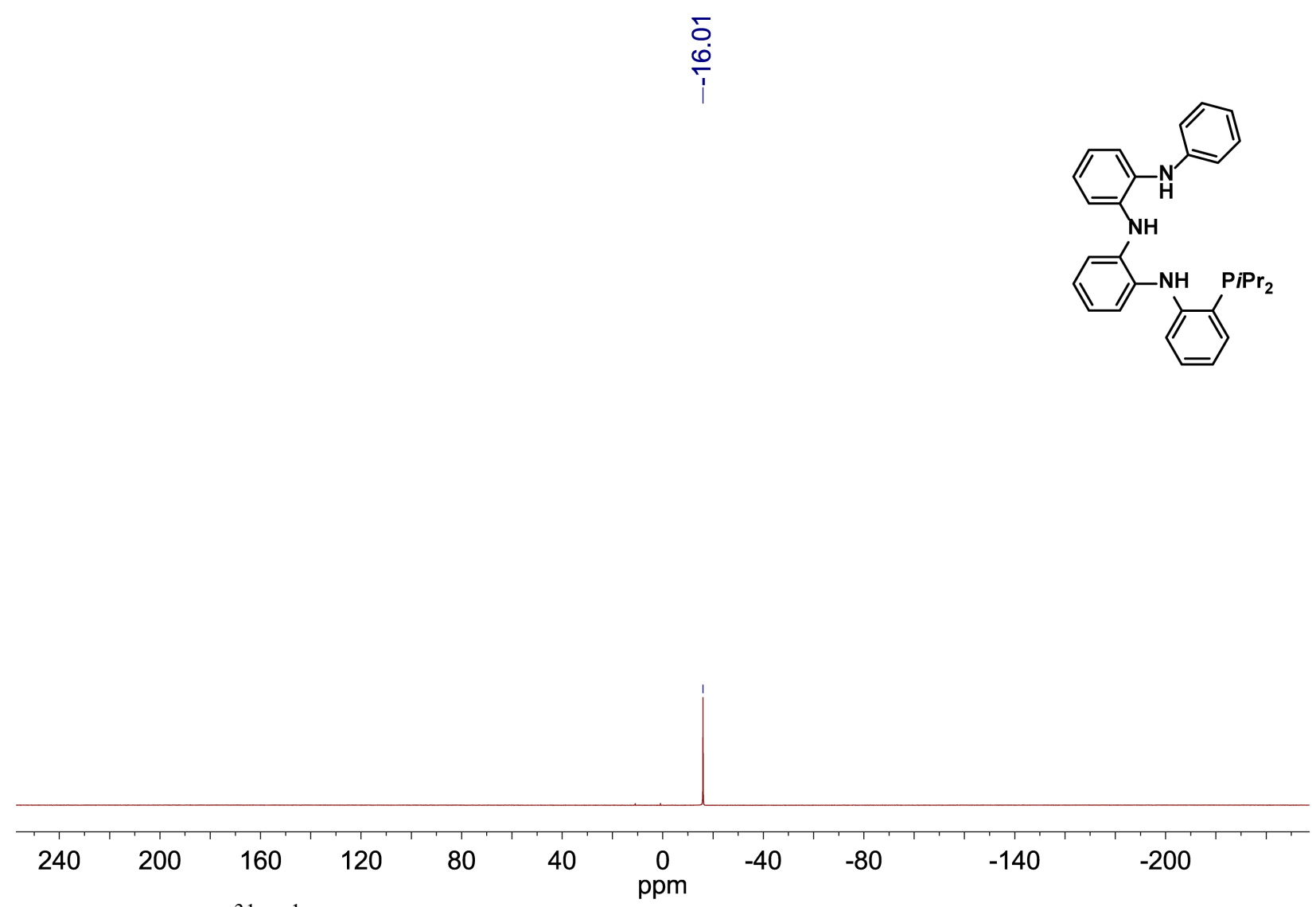

Figure S7. ${ }^{31} \mathrm{P}\left\{{ }^{1} \mathrm{H}\right\} \quad$ NMR spectrum of N1-(2-diisopropylphosphinophenyl)-N2-(2(phenylamino)phenyl)benzene-1,2-diamine (2) recorded in $\mathrm{CDCl}_{3}-\mathrm{d}_{1}$ at $23{ }^{\circ} \mathrm{C}$. 

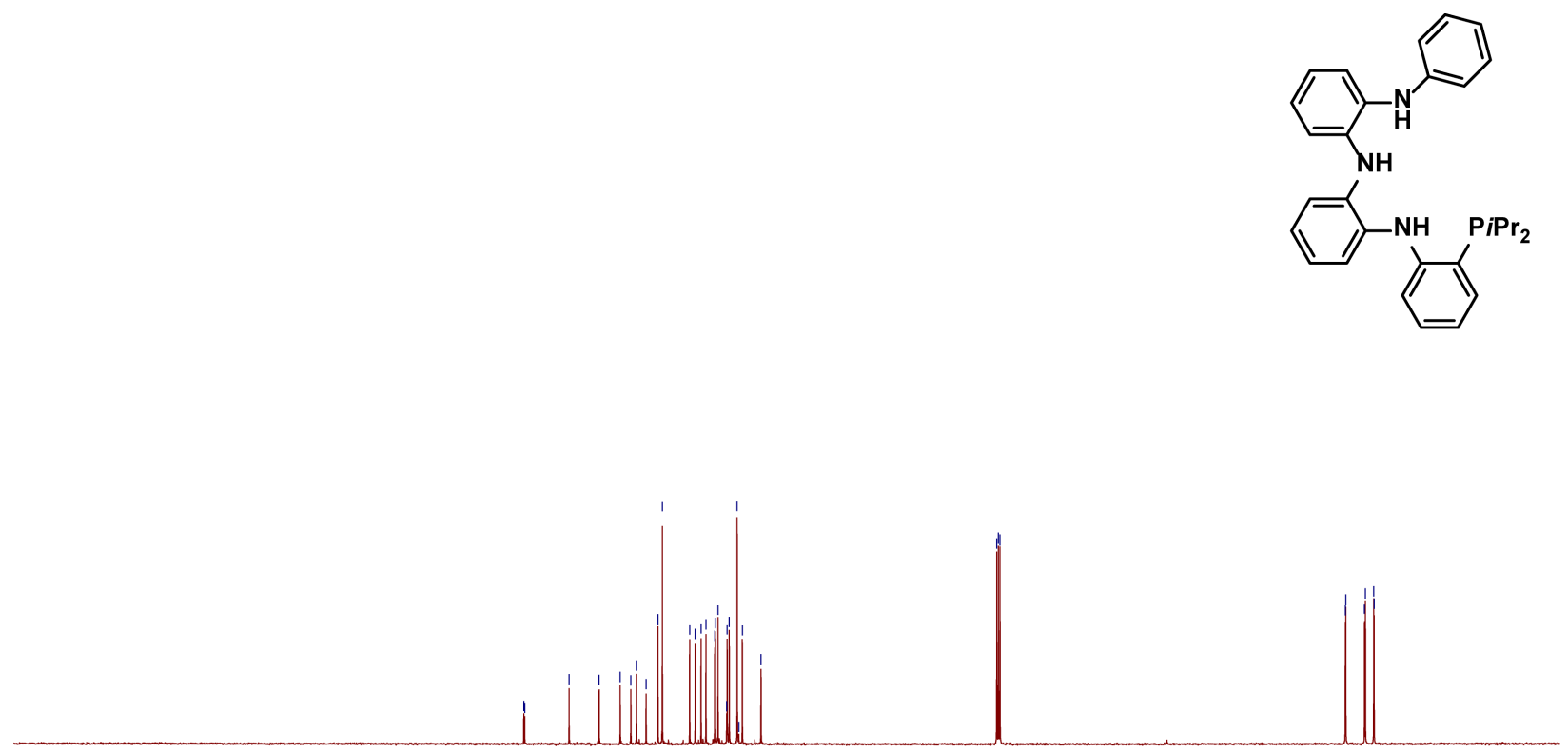

100

80

60

40

20

of

N1-(2-diisopropylphosphinophenyl)-N2-(2- 


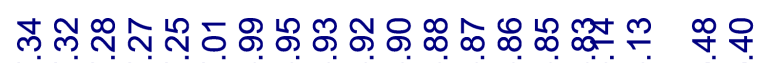

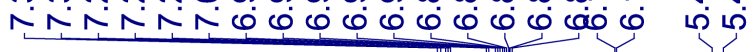
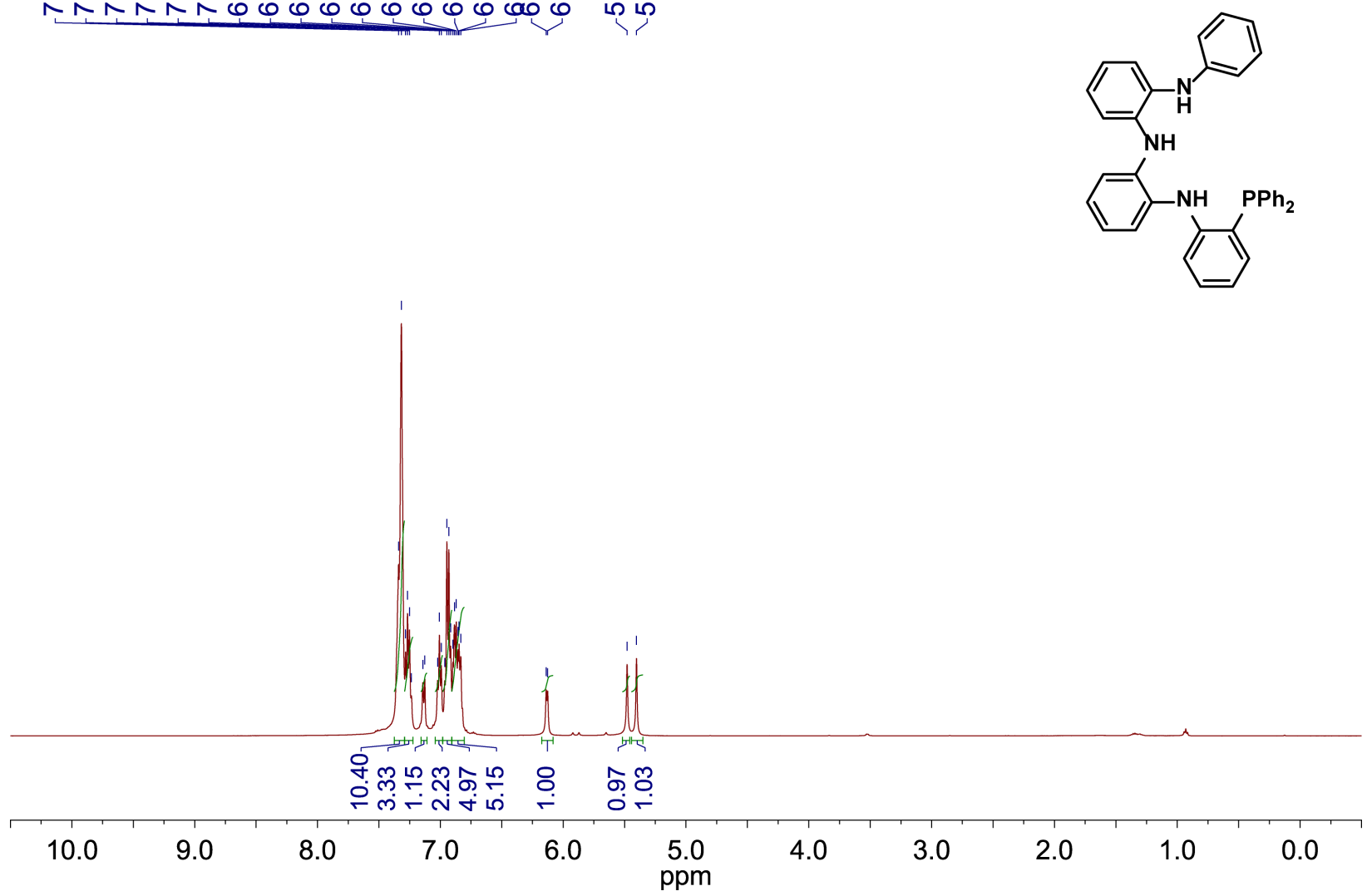

Figure S9. $\quad{ }^{1} \mathrm{H} \quad$ NMR $\quad$ spectrum of N1-(2-diphenylphosphinophenyl)-N2-(2(phenylamino)phenyl)benzene-1,2-diamine (3) recorded in $\mathrm{CDCl}_{3}-\mathrm{d}_{1}$ at $23{ }^{\circ} \mathrm{C}$. 
$\underset{\substack{+ \\ \hdashline}}{\infty}$
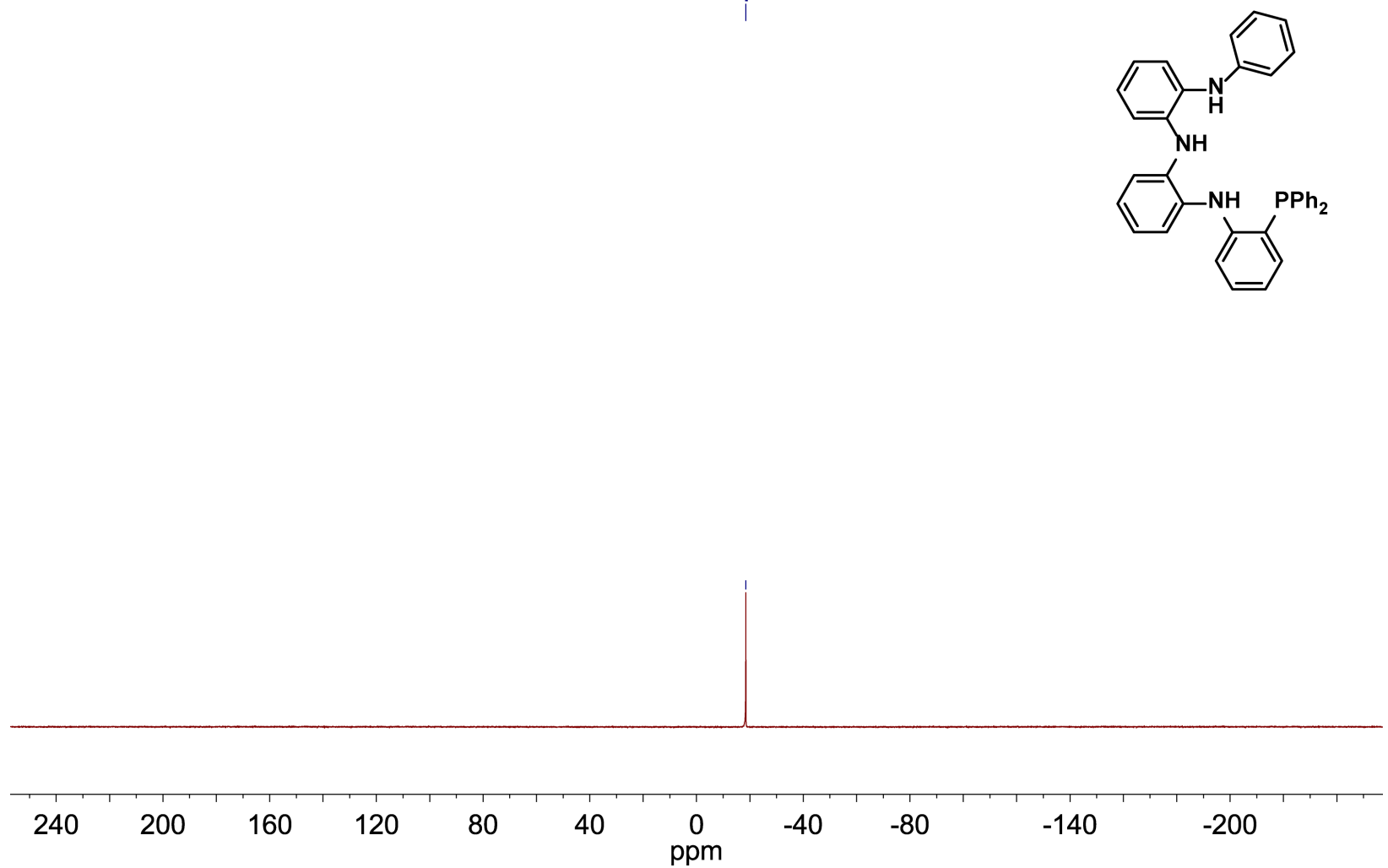

Figure S10. ${ }^{31} \mathrm{P}\left\{{ }^{1} \mathrm{H}\right\} \quad$ NMR spectrum of N1-(2-diphenylphosphinophenyl)-N2-(2(phenylamino)phenyl)benzene-1,2-diamine (3) recorded in $\mathrm{CDCl}_{3}-\mathrm{d}_{1}$ at $23{ }^{\circ} \mathrm{C}$. 


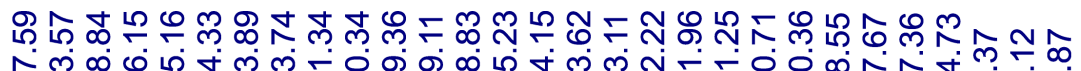

守宁宁宁

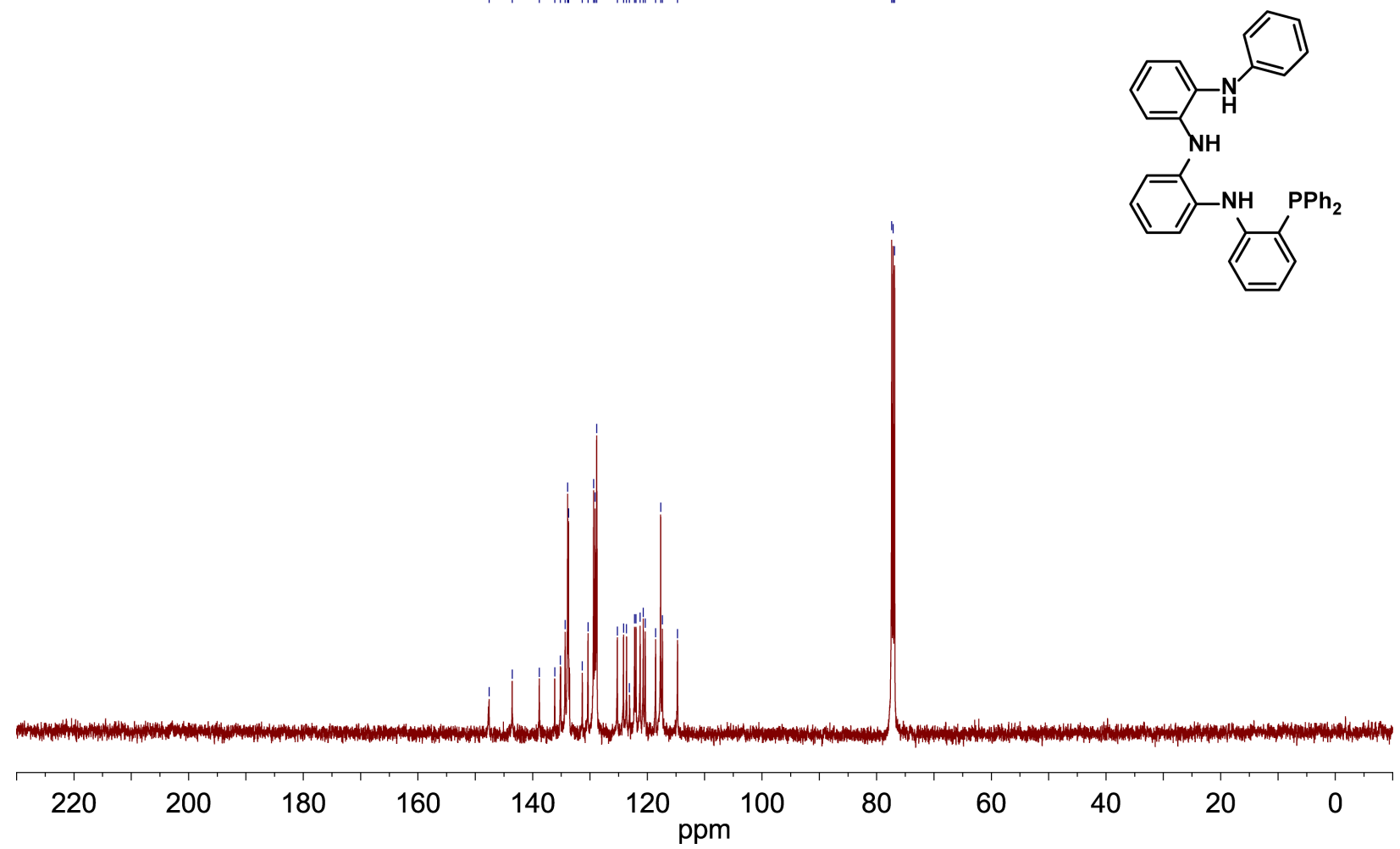

Figure S11. ${ }^{13} \mathrm{C}$ NMR spectrum of N1-(2-diphenylphosphinophenyl)-N2-(2(phenylamino)phenyl)benzene-1,2-diamine (3) recorded in $\mathrm{CDCl}_{3}-\mathrm{d}_{1}$ at $23{ }^{\circ} \mathrm{C}$. 


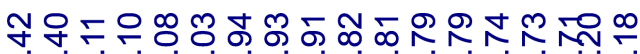

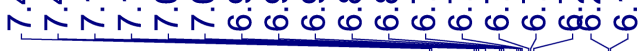

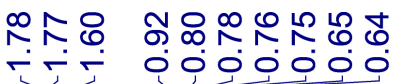
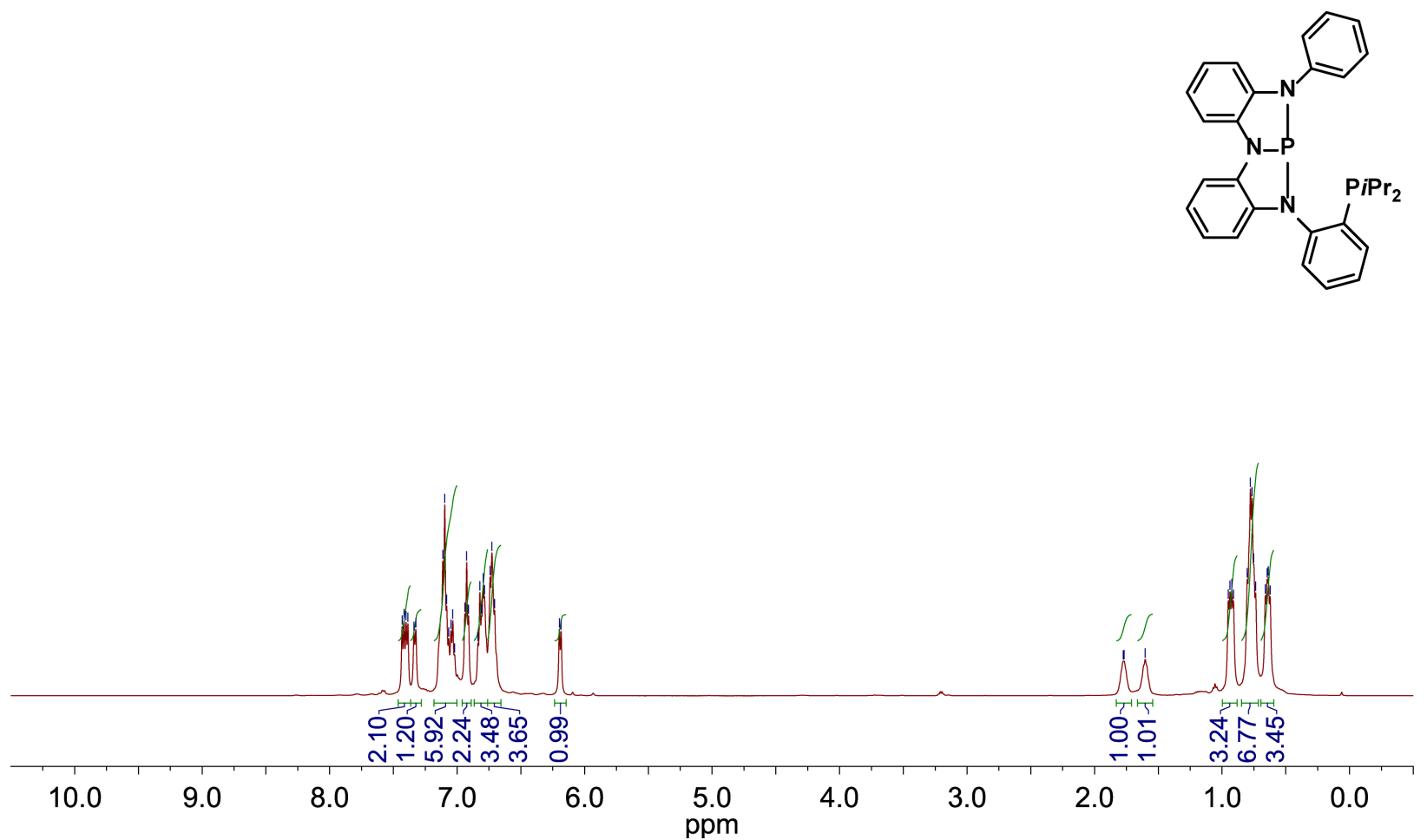

Figure S12. ${ }^{1} \mathrm{H}$ NMR spectrum of $\mathbf{L 1}$ recorded in $\mathrm{C}_{6} \mathrm{D}_{6}-\mathrm{d}_{6}$ at $23{ }^{\circ} \mathrm{C}$. 

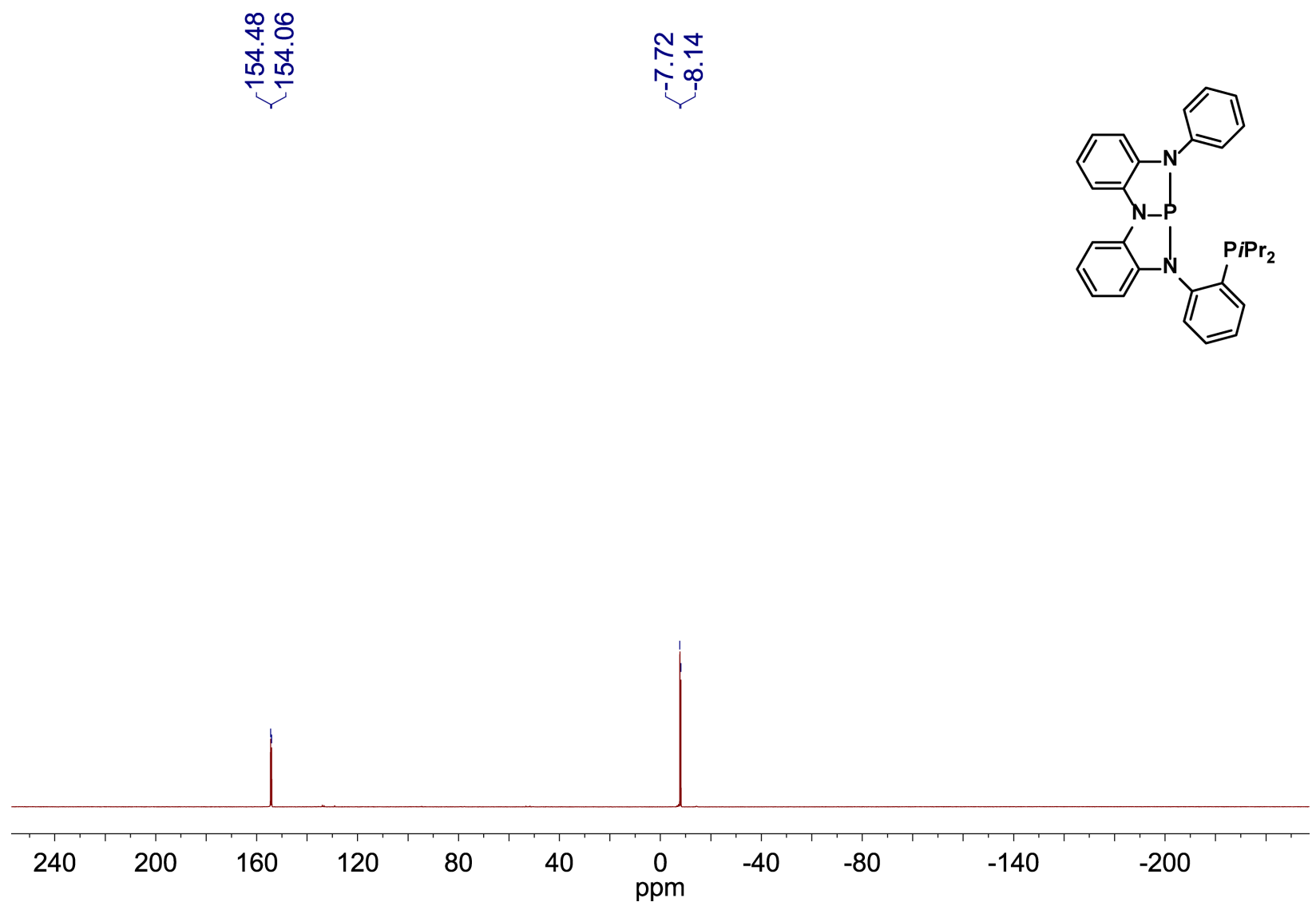

Figure S13. ${ }^{31} \mathrm{P}\left\{{ }^{1} \mathrm{H}\right\}$ NMR spectrum of $\mathbf{L 1}$ recorded in $\mathrm{C}_{6} \mathrm{D}_{6}-\mathrm{d}_{6}$ at $23{ }^{\circ} \mathrm{C}$. 


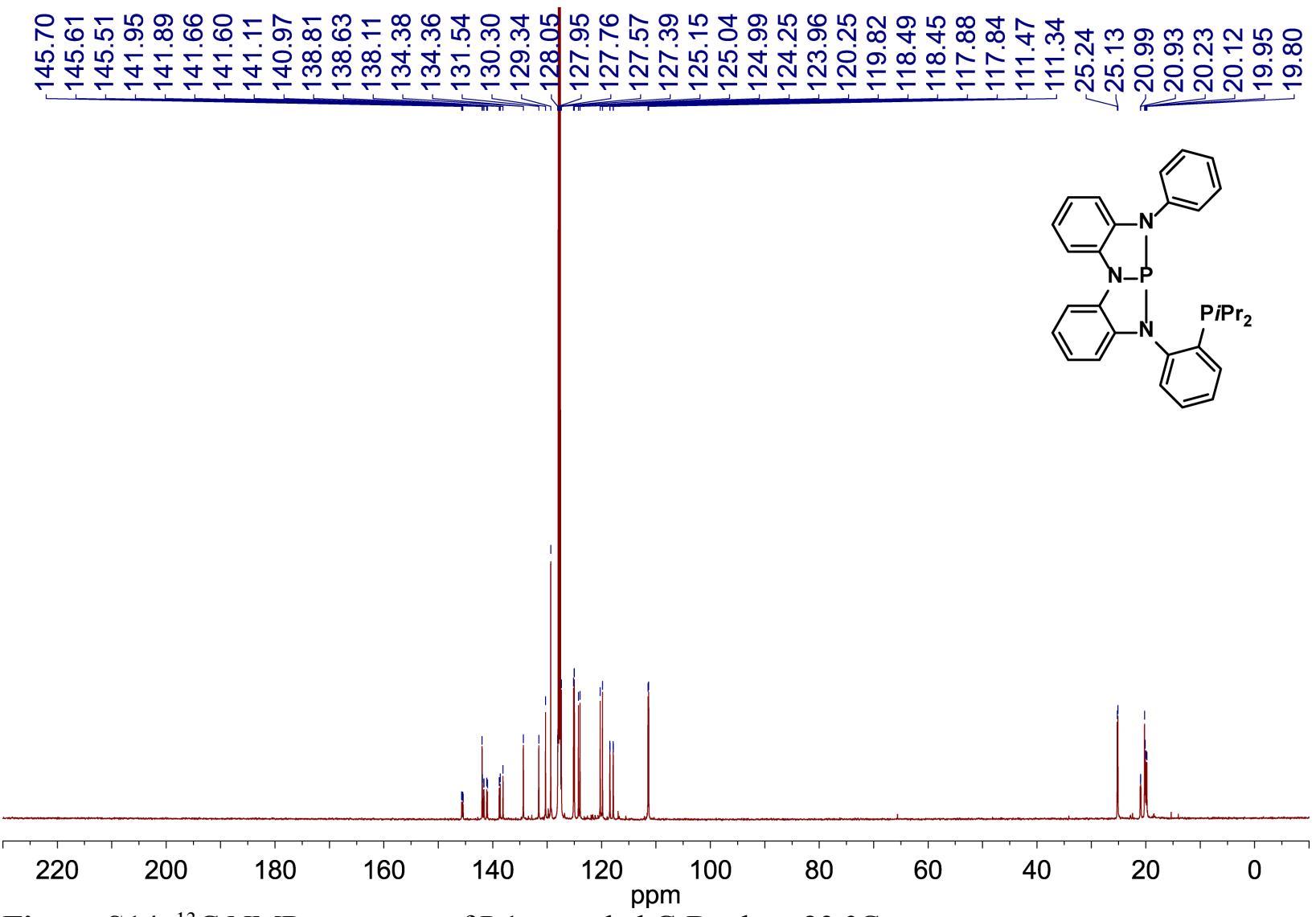

Figure S14. ${ }^{13} \mathrm{C}$ NMR spectrum of $\mathbf{L 1}$ recorded $\mathrm{C}_{6} \mathrm{D}_{6}-\mathrm{d}_{6}$ at $23{ }^{\circ} \mathrm{C}$. 


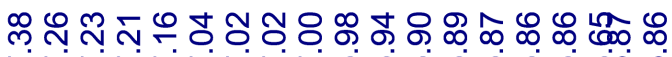

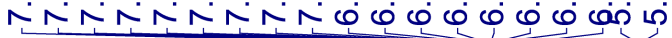
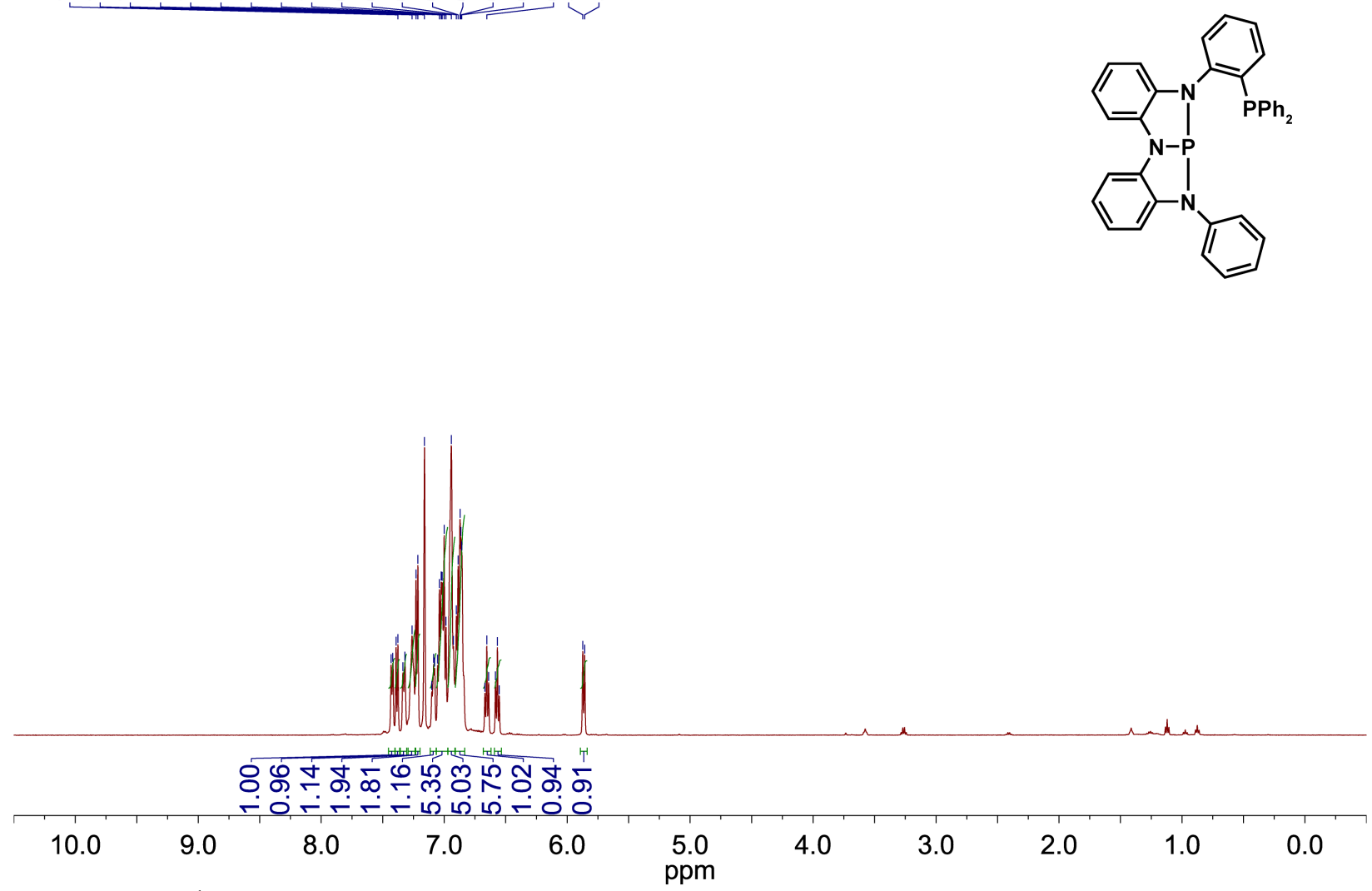

Figure S15. ${ }^{1} \mathrm{H}$ NMR spectrum of $\mathbf{L 2}$ recorded in $\mathrm{C}_{6} \mathrm{D}_{6-} \mathrm{d}_{6}$ at $23{ }^{\circ} \mathrm{C}$. Asterisk symbol (*) denotes residual ${ }^{1} \mathrm{H}$ peak of small amounts of $\mathrm{Et}_{2} \mathrm{O}$ and pentane from crystallization. 

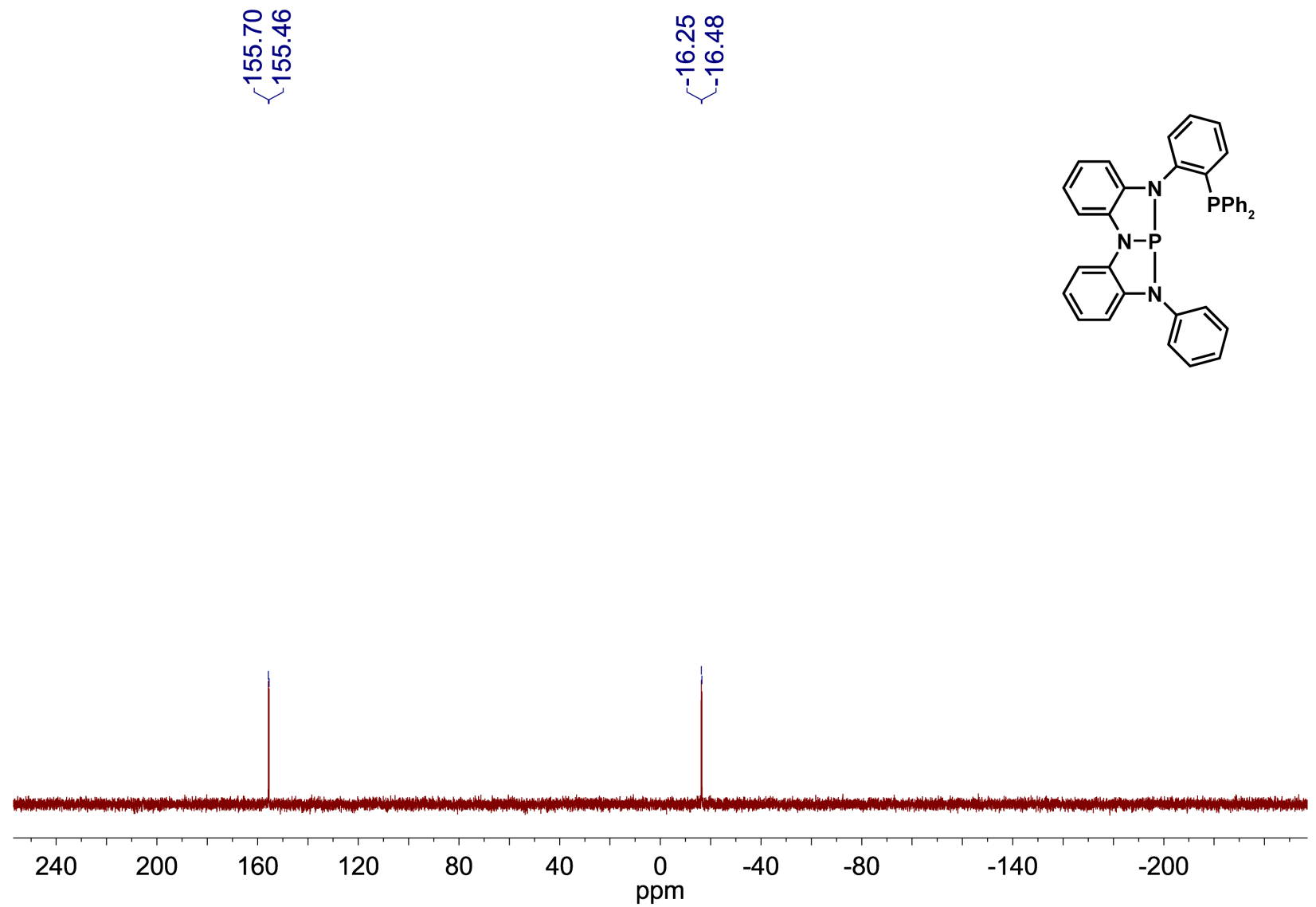

Figure S16. ${ }^{31} \mathrm{P}\left\{{ }^{1} \mathrm{H}\right\}$ NMR spectrum of $\mathbf{L} 2$ recorded in $\mathrm{C}_{6} \mathrm{D}_{6}-\mathrm{d}_{6}$ at $23{ }^{\circ} \mathrm{C}$. 


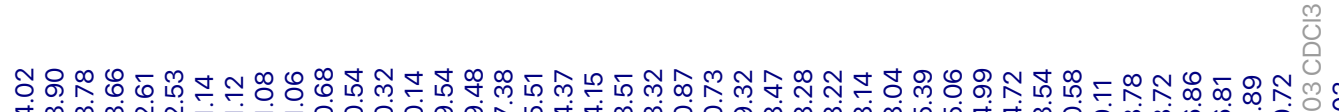

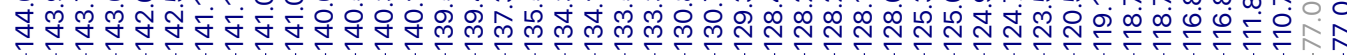
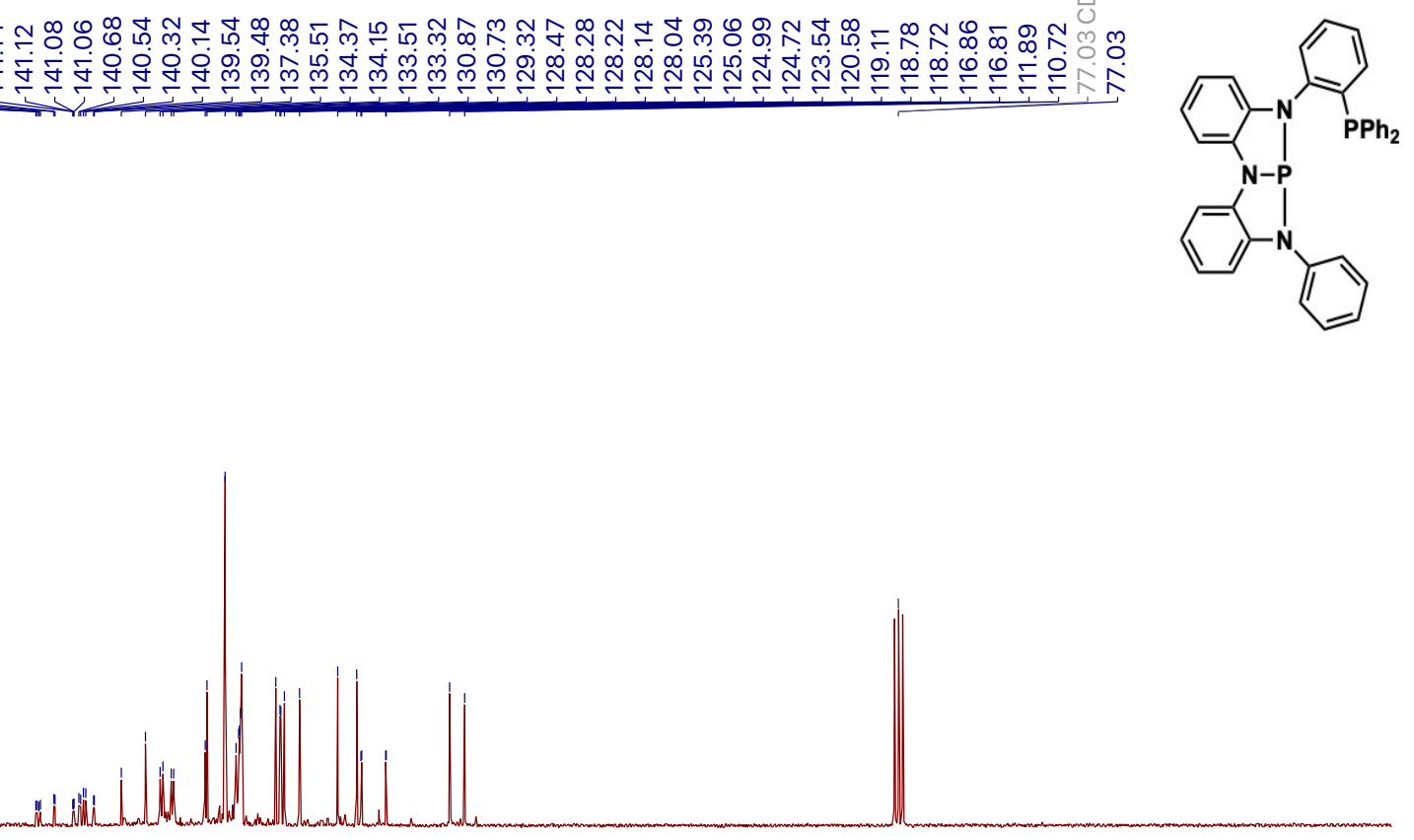

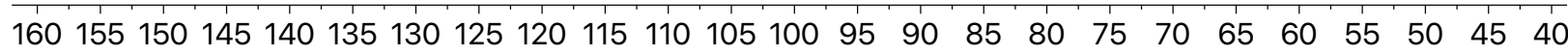
ppm

Figure S17. ${ }^{13} \mathrm{C}$ NMR spectrum of $\mathbf{L 2}$ recorded in $\mathrm{CDCl}_{3}-\mathrm{d}_{1}$ at $23{ }^{\circ} \mathrm{C}$. 


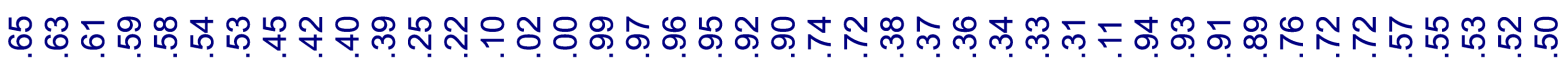

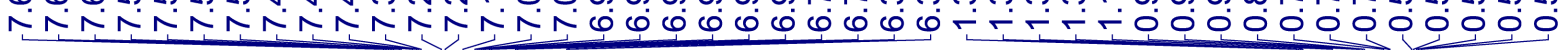
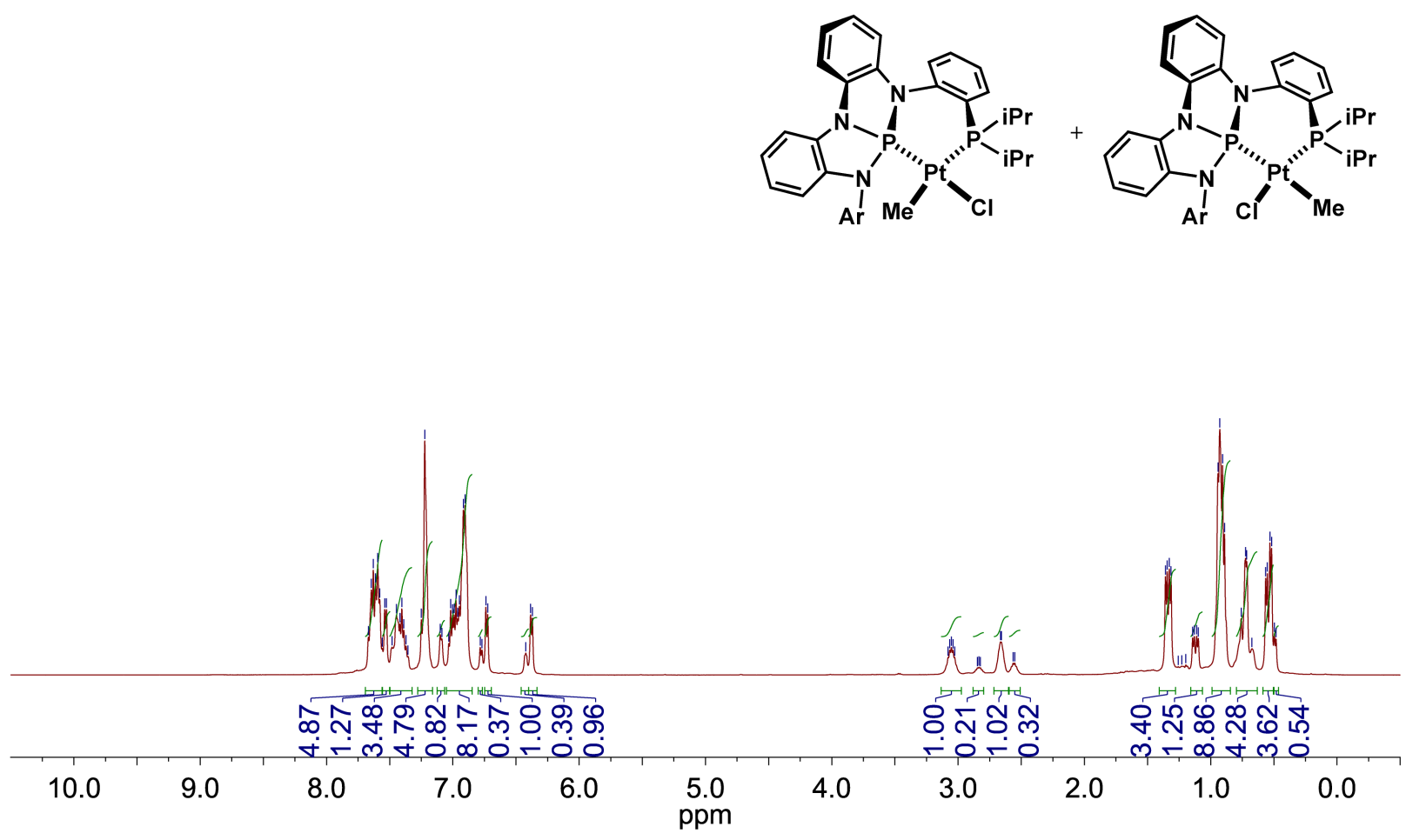

Figure S18. ${ }^{1} \mathrm{H}$ NMR spectrum of $\mathrm{PtClMeiPr}_{2} \mathrm{PNNNP}(4)$ recorded in $\mathrm{CDCl}_{3}-\mathrm{d}_{1}$ at $23{ }^{\circ} \mathrm{C}$. 


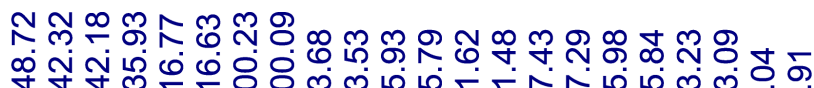

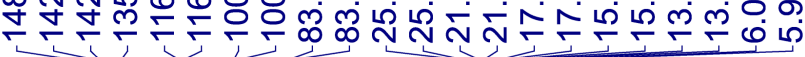

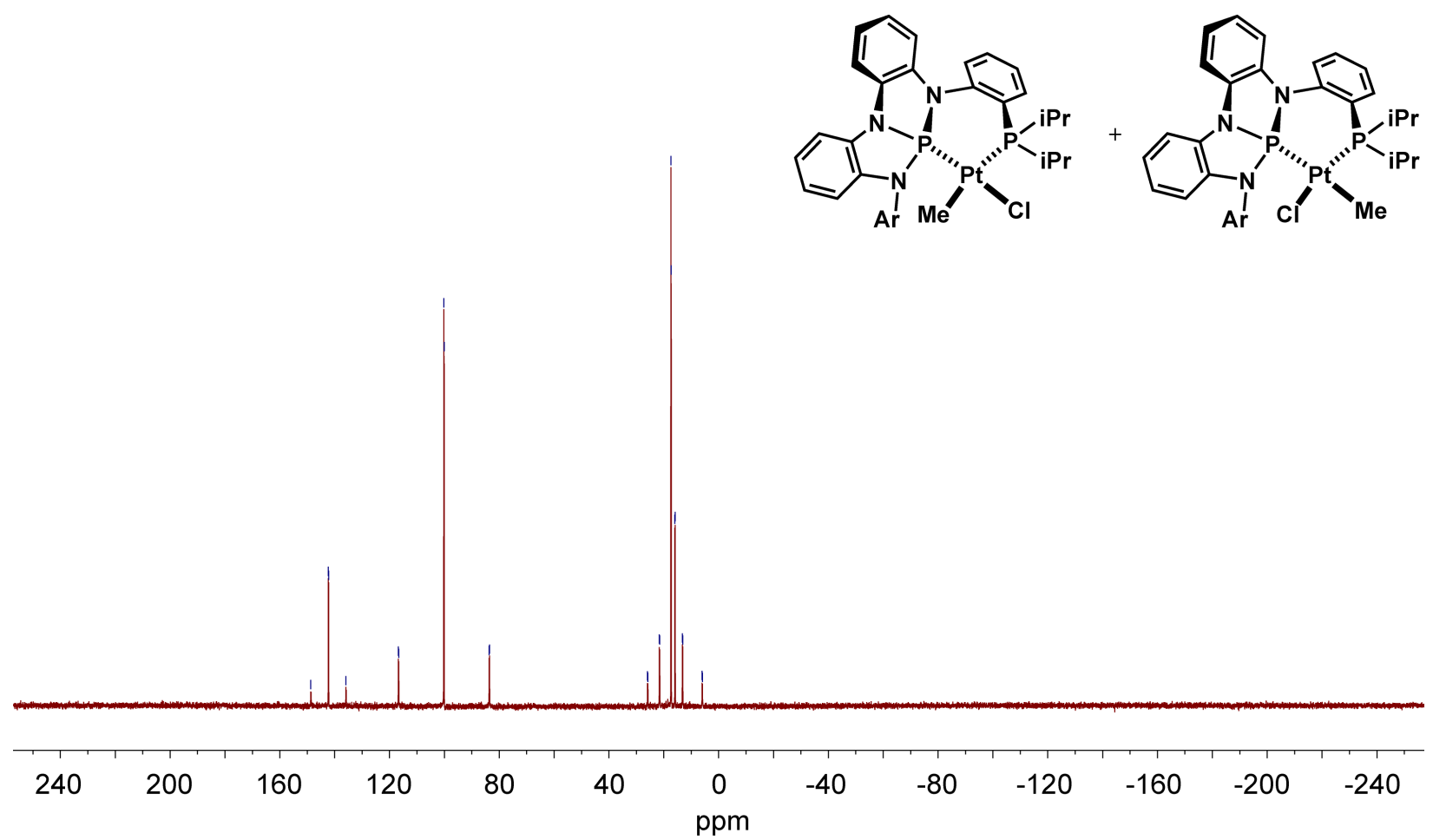

Figure S19. ${ }^{31} \mathrm{P}\left\{{ }^{1} \mathrm{H}\right\}$ NMR spectrum of $\mathrm{PtClMeiPr}_{2} \mathrm{PNNNP}(4)$ recorded in $\mathrm{CDCl}_{3}-\mathrm{d}_{1}$ at $23{ }^{\circ} \mathrm{C}$. 


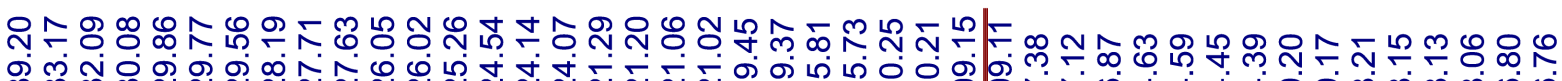
স্户

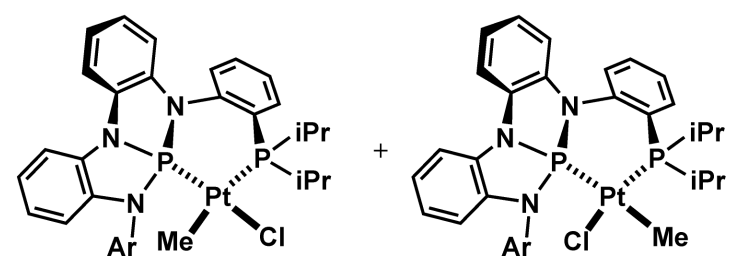

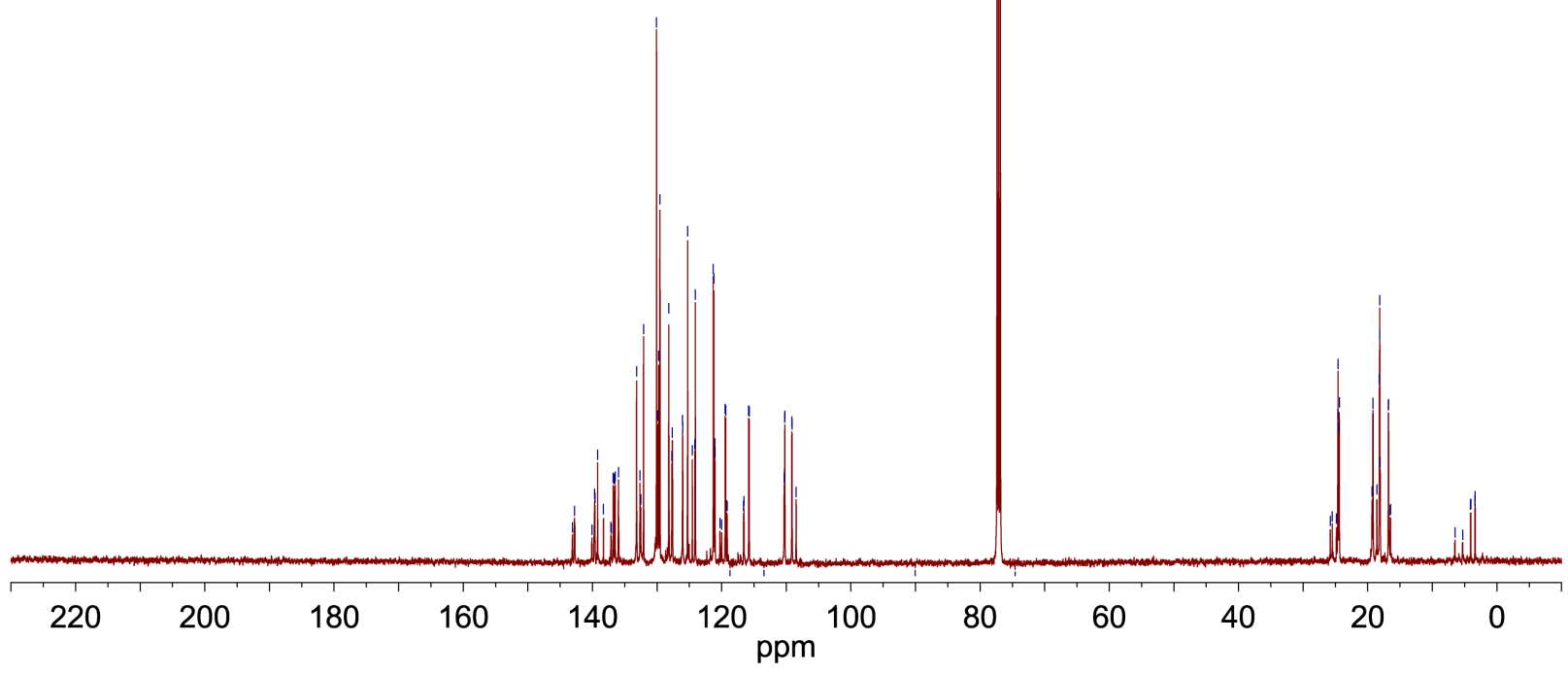

Figure S20. ${ }^{13} \mathrm{C}$ NMR spectrum of $\mathrm{PtClMeiPr}_{2} \mathrm{PNNNP}(4)$ recorded in $\mathrm{CDCl}_{3}-\mathrm{d}_{1}$ at $23{ }^{\circ} \mathrm{C}$. 


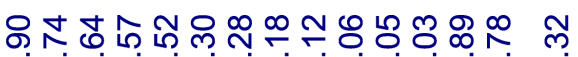

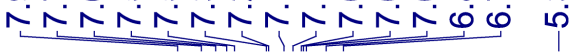
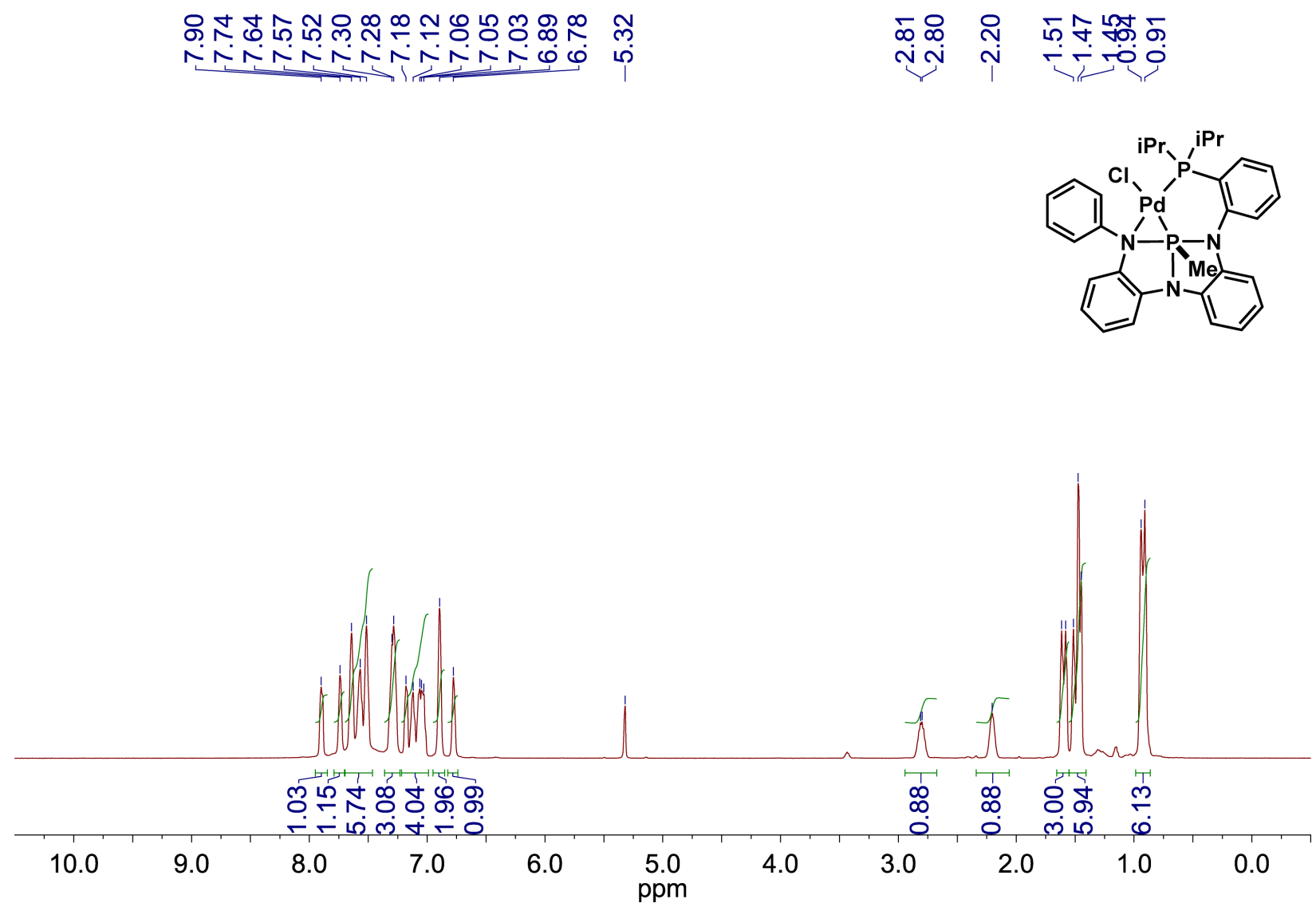

Figure S21. ${ }^{1} \mathrm{H}$ NMR spectrum of $\mathrm{PdCliPr}_{2} \mathrm{PNNNPMe}\left(\mathbf{5}^{\prime}\right)$ recorded in $\mathrm{CD}_{2} \mathrm{Cl}_{2}-\mathrm{d}_{2}$ at $23{ }^{\circ} \mathrm{C}$. 


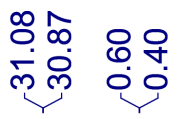
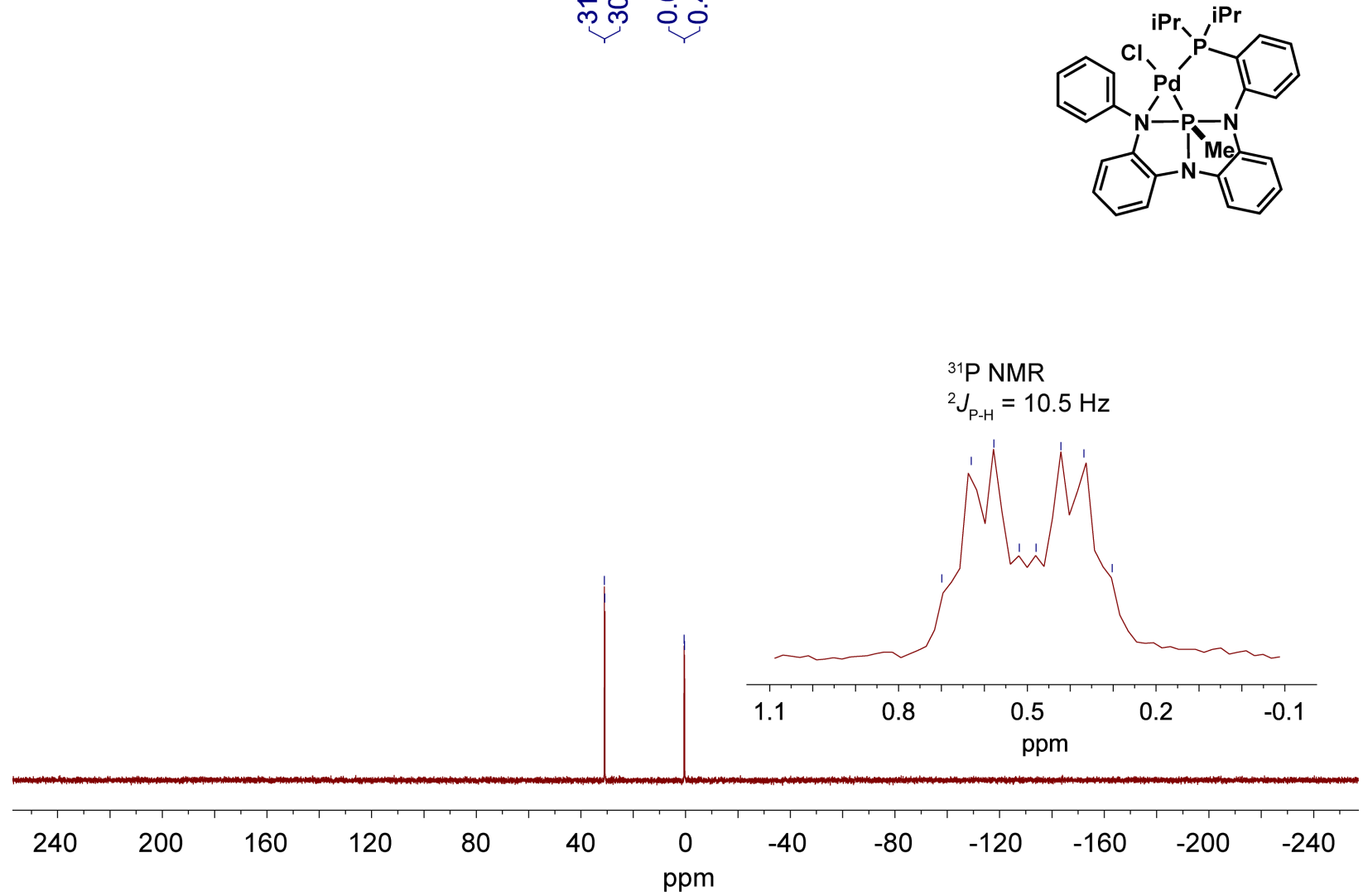

Figure S22. ${ }^{31} \mathrm{P}$ NMR spectra of $\mathrm{PdCliPr}_{2} \mathrm{PNNNPMe}\left(\mathbf{5}^{\prime}\right)$ recorded in $\mathrm{CD}_{2} \mathrm{Cl}_{2}-\mathrm{d}_{2}$ at $23{ }^{\circ} \mathrm{C}$. 


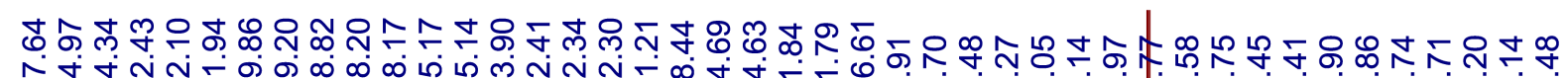
守崩岕

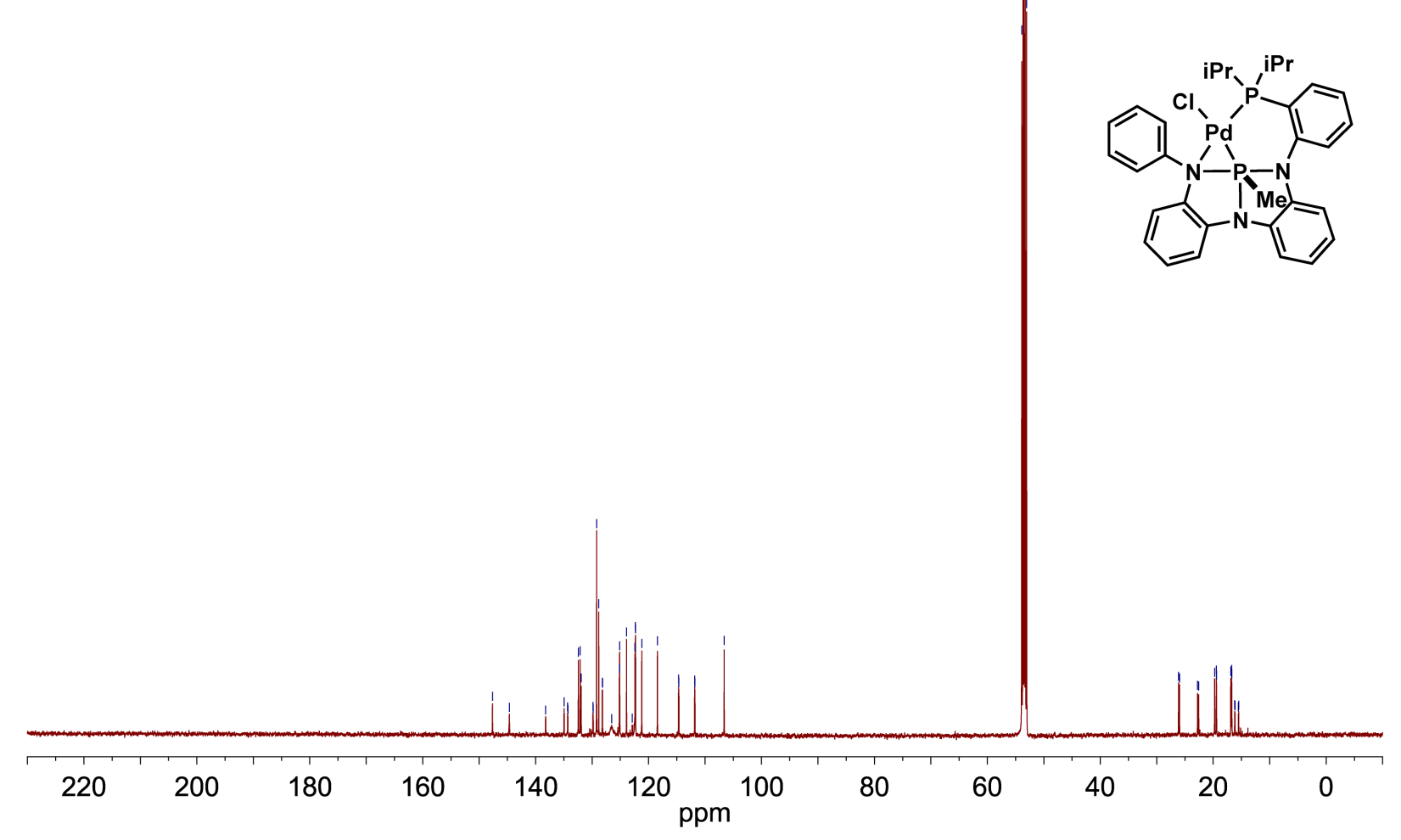

Figure S23. ${ }^{13} \mathrm{C}$ NMR spectrum of $\mathrm{PdCliPr}_{2} \mathrm{PNNNPMe}\left(5^{\prime}\right)$ recorded in $\mathrm{CD}_{2} \mathrm{Cl}_{2}-\mathrm{d}_{2}$ at $23{ }^{\circ} \mathrm{C}$. 


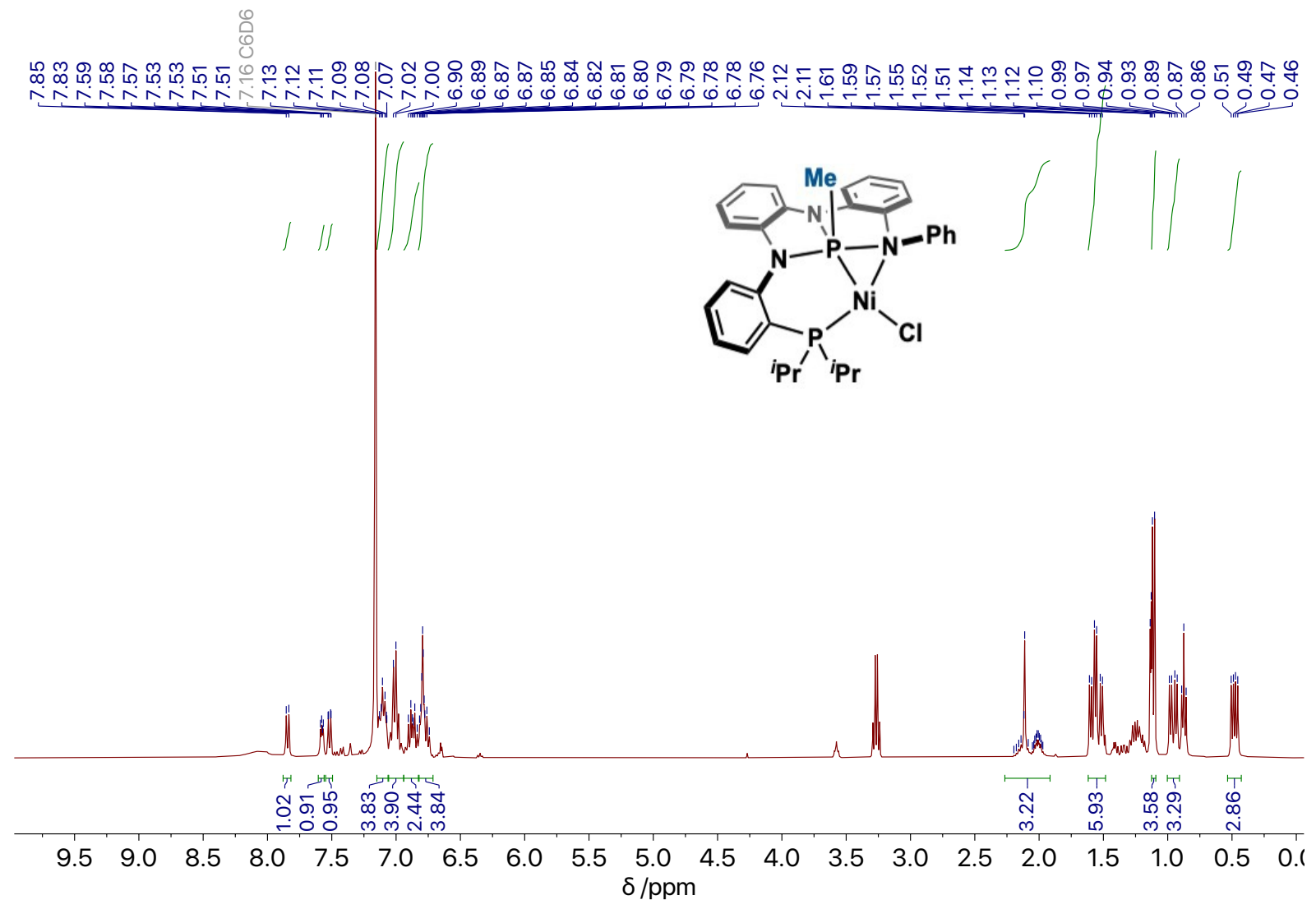

Figure S24. ${ }^{1} \mathrm{H}$ NMR spectrum of $\mathrm{NiCliPr}_{2} \mathrm{PNNNPMe}\left(6^{\prime}\right)$ recorded in $\mathrm{C}_{6} \mathrm{D}_{6}-\mathrm{d}_{6}$ at $23{ }^{\circ} \mathrm{C}$. 

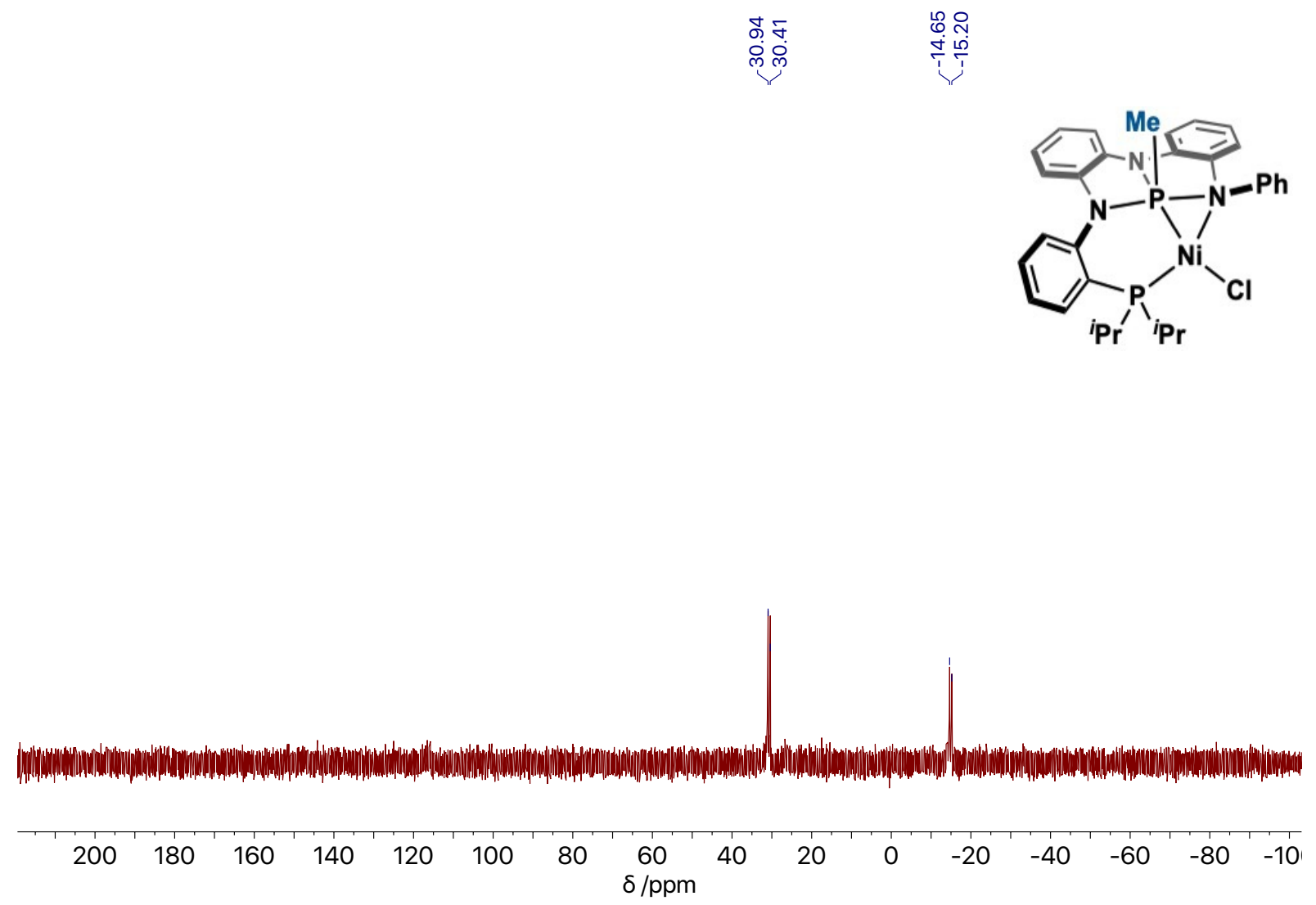

Figure S25. ${ }^{31} \mathrm{P}\left\{{ }^{1} \mathrm{H}\right\}$ NMR spectrum of $\mathrm{NiCliPr} 2 \mathrm{PNNNPMe}\left(6^{\prime}\right)$ recorded in $\mathrm{CD}_{2} \mathrm{Cl}_{2}-\mathrm{d}_{2}$ at $23{ }^{\circ} \mathrm{C}$. 


\section{X-Ray Data Analysis \\ C.1. Structural Data for $L 2$}

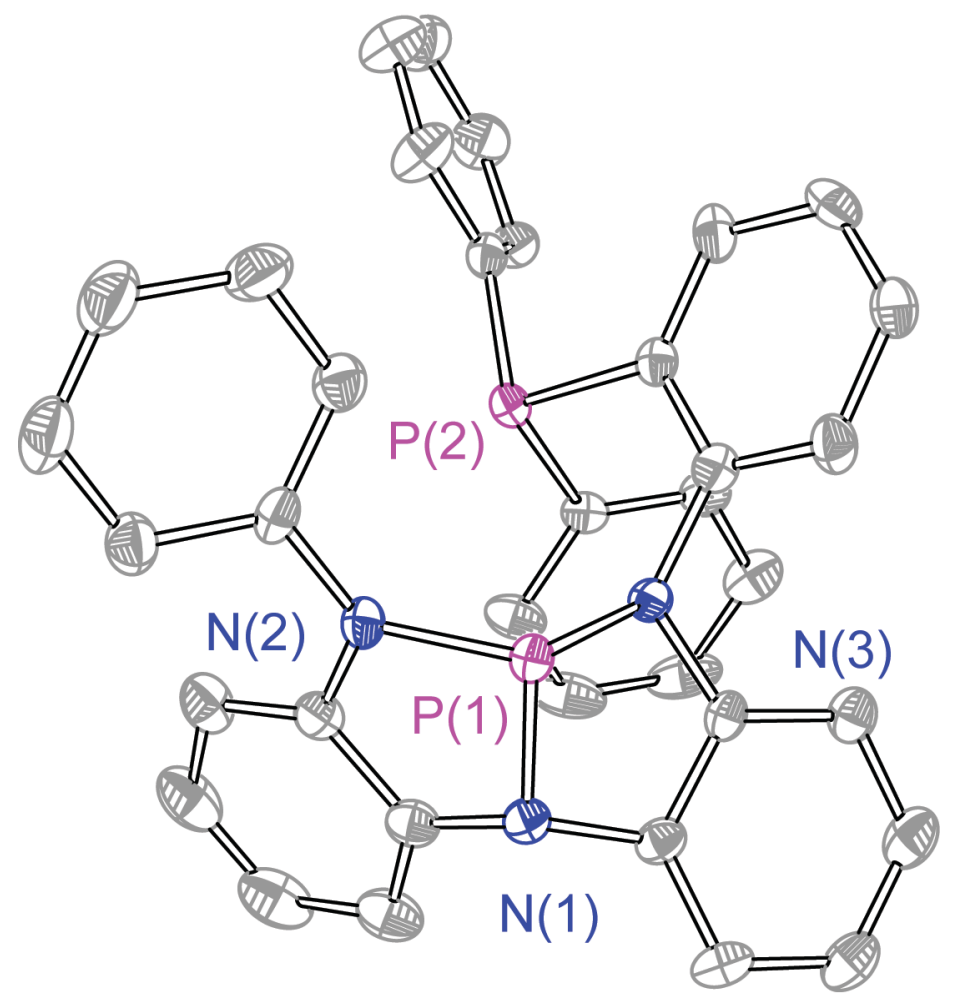

Figure S26. Thermal ellipsoid plots of $\kappa^{2}$-chelating ligand (L2) drawn at the $50 \%$ probability level. $\mathrm{H}$-atoms and solvent are omitted for clarity. 
Table S1. X-ray experimental details for complex L2 (CCDC 2033415)

\begin{tabular}{|c|c|}
\hline \multicolumn{2}{|l|}{ Crystal Data } \\
\hline Chemical formula & $\mathrm{C}_{40} \mathrm{H}_{37} \mathrm{~N}_{3} \mathrm{OP}_{2}$ \\
\hline Fw, g/mol & 637.66 \\
\hline Crystal system, space group & monoclinic, $P 2_{1}$ \\
\hline Temperature $(\mathrm{K})$ & $100(2)$ \\
\hline$a, b, c(\AA)$ & $18.9534(9), 8.4688(4), 21.9113(11)$ \\
\hline$\alpha, \beta, \gamma\left(^{\circ}\right)$ & $90,107.1402(18), 90$ \\
\hline$V\left(\AA^{3}\right)$ & $3360.8(3)$ \\
\hline$Z$ & 4 \\
\hline Radiation type & Mo $K \alpha$ \\
\hline$\mu\left(\mathrm{mm}^{-1}\right)$ & 0.166 \\
\hline Crystal size $(\mathrm{mm})$ & $0.350 \times 0.105 \times 0.040$ \\
\hline \multicolumn{2}{|l|}{ Data collection } \\
\hline Diffractometer & Bruker Photon2 CPAD \\
\hline Absorption correction & Multi-scan, $S A D A B S$ \\
\hline$T_{\min }, T_{\max }$ & $0.6610,0.7214$ \\
\hline $\begin{array}{l}\text { No. of measured, independent and observed } \\
{[I>2 \sigma(I)] \text { reflections }}\end{array}$ & $245534,19638,14985$ \\
\hline $\begin{array}{l}R_{\text {int }} \\
(\sin \theta / \lambda)_{\max }\left(\AA^{-1}\right)\end{array}$ & $\begin{array}{l}0.1159 \\
0.600\end{array}$ \\
\hline \multicolumn{2}{|l|}{ Refinement } \\
\hline$R\left[F^{2}>2 \sigma\left(F^{2}\right)\right], w R\left(F^{2}\right), S$ & $0.0942,0.2307,1.071$ \\
\hline No. of reflections & 19638 \\
\hline No. of parameters & 1339 \\
\hline No. of restraints & 0 \\
\hline $\mathrm{H}$-atom treatment & $\begin{array}{l}\mathrm{H} \text { atoms treated by a mixture of independent } \\
\text { and constrained refinement }\end{array}$ \\
\hline $\operatorname{Dr}_{\max }, \operatorname{Dr}_{\min }\left(\mathrm{e} \AA^{-3}\right)$ & $1.029,-2.297$ \\
\hline
\end{tabular}




\section{C.2. Structural Data for PtCIMeiPr2 2 PNNP (4)}

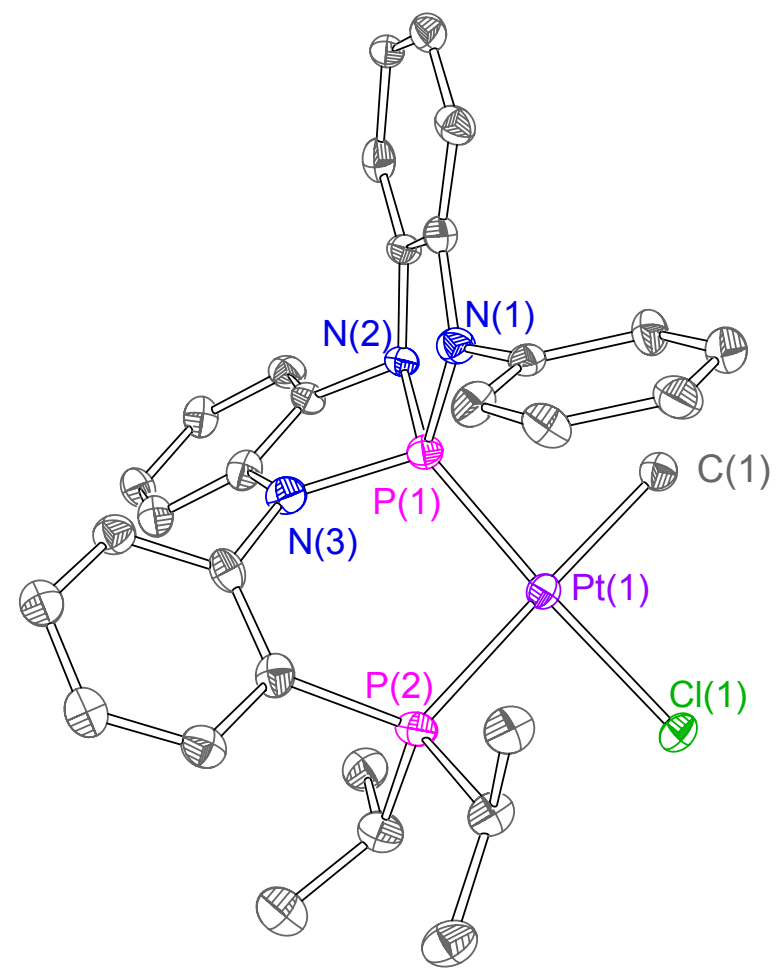

Figure S27. Thermal ellipsoid plots of $\mathrm{PtClMeiPr}_{2} \mathrm{PNNNP}$ (4) drawn at the 50\% probability level. H-atoms are omitted for clarity. 
Table S2. X-ray experimental details for complex 4 (CCDC 2036533)

\begin{tabular}{|c|c|}
\hline \multicolumn{2}{|l|}{ Crystal Data } \\
\hline Chemical formula & $\mathrm{C}_{62} \mathrm{H}_{68} \mathrm{Cl}_{2} \mathrm{~N}_{6} \mathrm{P}_{4} \mathrm{Pt}_{2}$ \\
\hline Fw, g/mol & 1482.18 \\
\hline Crystal system, space group & Monoclinic, $P 2(1) / c$ \\
\hline Temperature $(\mathrm{K})$ & $15(2)$ \\
\hline$a, b, c(\AA)$ & $21.249(3), 18.319(2), 14.6841(18)$ \\
\hline$\alpha, \beta, \gamma\left({ }^{\circ}\right)$ & $90,101.7720(10), 90$ \\
\hline$V\left(\AA^{3}\right)$ & $5595.7(12)$ \\
\hline$Z$ & 4 \\
\hline Radiation type & synchrotron \\
\hline$\mu\left(\mathrm{mm}^{-1}\right)$ & 0.297 \\
\hline Crystal size (mm) & $0.01 \times 0.01 \times 0.01$ \\
\hline \multicolumn{2}{|l|}{ Data collection } \\
\hline Diffractometer & Bruker $A P E X$-II CCD \\
\hline Absorption correction & Multi-scan, $S A D A B S$ \\
\hline$T_{\min }, T_{\max }$ & $0.9970,0.9970$ \\
\hline $\begin{array}{l}\text { No. of measured, independent and observed } \\
{[I>2 \sigma(I)] \text { reflections }}\end{array}$ & $97120,10021,9299$ \\
\hline$R_{\text {int }}$ & 0.0475 \\
\hline$(\sin \theta / \lambda)_{\max }\left(\AA^{-1}\right)$ & 0.597 \\
\hline \multicolumn{2}{|l|}{ Refinement } \\
\hline$R\left[F^{2}>2 \sigma\left(F^{2}\right)\right], w R\left(F^{2}\right), S$ & $0.0463,0.1258,0.969$ \\
\hline No. of reflections & 10021 \\
\hline No. of parameters & 718 \\
\hline No. of restraints & 12 \\
\hline H-atom treatment & $\begin{array}{l}\mathrm{H} \text { atoms treated by a mixture of independent } \\
\text { and constrained refinement }\end{array}$ \\
\hline $\mathrm{Dr}_{\max }, \operatorname{Dr}_{\min }\left(\mathrm{e} \AA^{-3}\right)$ & $0.328,-0.270$ \\
\hline
\end{tabular}




\section{C.3. Structural Data for PdCliPr 2 PNNNPMe (5')}

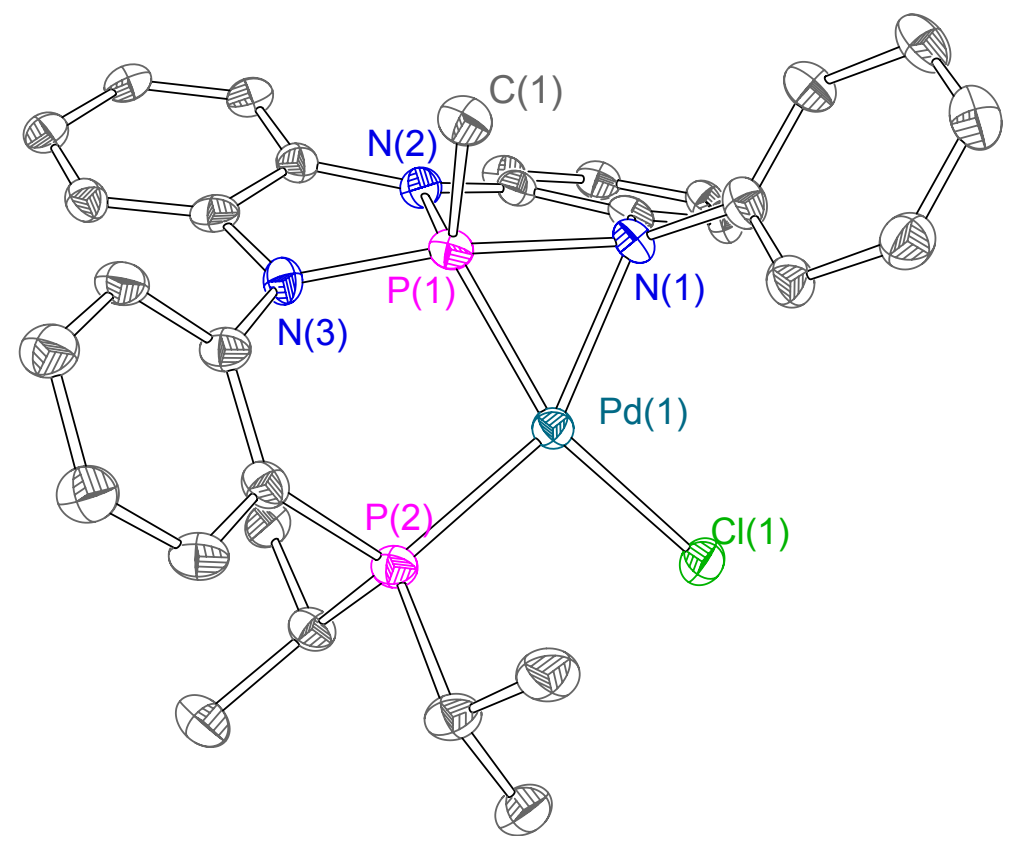

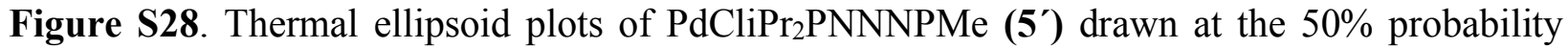
level. H-atoms and solvent are omitted for clarity. 
Table S3. X-ray experimental details for complex 5' (CCDC 2033417)

\section{Crystal Data}

Chemical formula

Fw, g/mol

Crystal system, space group

Temperature $(\mathrm{K})$

$a, b, c(\AA)$

$\alpha, \beta, \gamma\left(^{\circ}\right)$

$V\left(\AA^{3}\right)$

$Z$

Radiation type

$\mu\left(\mathrm{mm}^{-1}\right)$

Crystal size (mm)

Data collection

Diffractometer

Absorption correction

$T_{\min }, T_{\max }$

No. of measured, independent and observed

$[I>2 \sigma(I)]$ reflections

$R_{\text {int }}$

$(\sin \theta / \lambda)_{\max }\left(\AA^{-1}\right)$

Refinement

$R\left[F^{2}>2 \sigma\left(F^{2}\right)\right], w R\left(F^{2}\right), S$

No. of reflections

No. of parameters

No. of restraints

H-atom treatment

$\operatorname{Dr}_{\max }, \operatorname{Dr}_{\min }\left(\mathrm{e} \AA^{-3}\right)$
$\mathrm{C}_{131} \mathrm{H}_{141} \mathrm{Cl}_{4} \mathrm{~F}_{3} \mathrm{~N}_{12} \mathrm{P}_{8} \mathrm{Pd}_{4}$

2755.71

tetragonal, $P-42(1) / c$

$15(2)$

15.426(3), 15.426(3), 26.835(6)

$90,90,90$

6386(3)

2

synchrotron

0.227

$0.20 \times 0.07 \times 0.07$

Bruker $A P E X$-II CCD

Multi-scan, $S A D A B S$

$0.956,0.984$

131592, 5631, 5168

0.0425

0.534

$0.0370,0.0969,1.087$

5631

396

0

$\mathrm{H}$ atoms treated by a mixture of independent and constrained refinement

$1.090,-0.998$ 


\section{C.4. Structural Data for NiCliPr 2 PNNNPMe (6')}

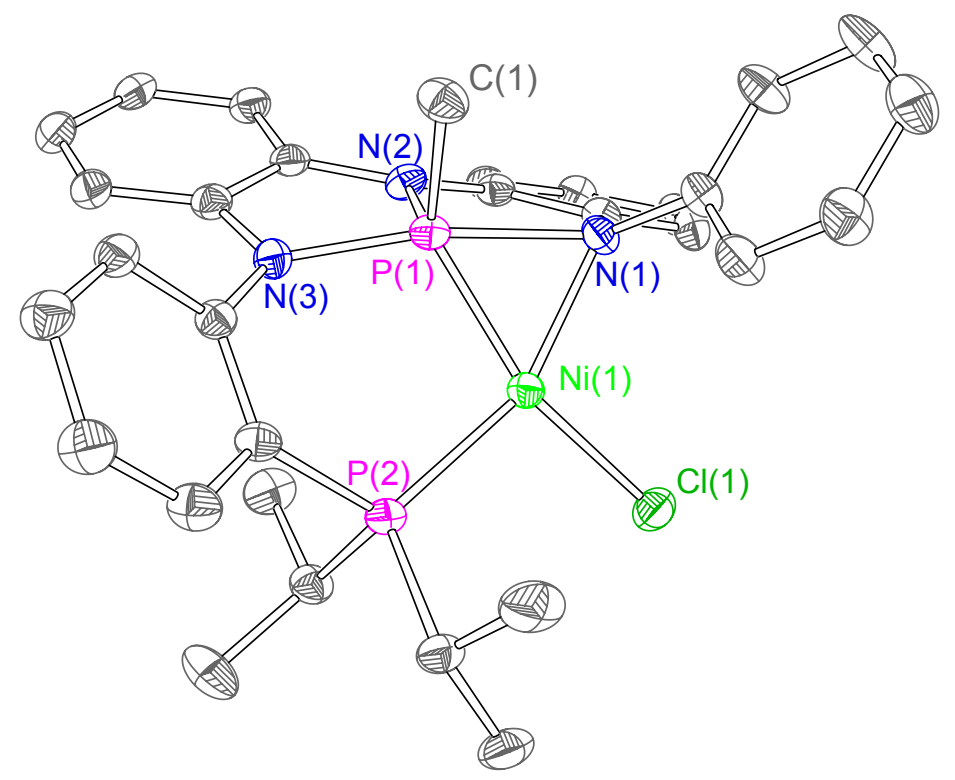

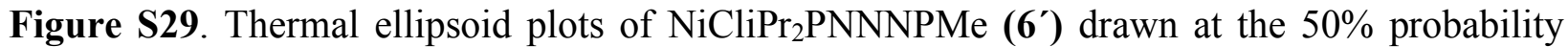
level. $\mathrm{H}$-atoms and solvent are omitted for clarity. 
Table S4. X-ray experimental details for complex 6' (CCDC 2033418)

\section{Crystal Data}

Chemical formula

Fw, g/mol

Crystal system, space group

Temperature (K)

$a, b, c(\AA)$

$\alpha, \beta, \gamma\left({ }^{\circ}\right)$

$V\left(\AA^{3}\right)$

$Z$

Radiation type

$\mu\left(\mathrm{mm}^{-1}\right)$

Crystal size (mm)

Data collection

Diffractometer

Absorption correction

$T_{\min }, T_{\max }$

No. of measured, independent and observed [ $I>$ $2 \sigma(I)]$ reflections

$R_{\text {int }}$

$(\sin \theta / \lambda)_{\max }\left(\AA^{-1}\right)$

Refinement

$R\left[F^{2}>2 \sigma\left(F^{2}\right)\right], w R\left(F^{2}\right), S$

No. of reflections

No. of parameters

No. of restraints

H-atom treatment

$\operatorname{Dr}_{\max }, \operatorname{Dr}_{\min }\left(\mathrm{e} \AA^{-3}\right)$
C33.75 H36 Cl N2 Ni P2

625.74

Tetragonal, $P-42(1) / c$

99.99

15.4791(13), 15.4791(13), 26.403(2)

$90,90,90$

6326.3(12)

8

Mo $K \alpha$

0.824

$0.285 \times 0.22 \times 0.015$

Bruker APEX-II CCD

Multi-scan, $S A D A B S$

$0.6820,0.7462$

10098

0.0601

0.710

$0.0523,0.1476,1.131$

10098

409

277

$\mathrm{H}$ atoms treated by a mixture of independent and constrained refinement

2.207, -0.607 
Table S5. Selected metrical parameters for compounds 1, 2, and L2.

\begin{tabular}{cccc} 
& $\mathbf{1}$ & $\mathbf{2}$ & $\mathbf{L 2}$ \\
\hline $\mathrm{P}(1)-\mathrm{N}(1)$ & $1.7610(12)$ & $1.7485(8)$ & $1.733(3)$ \\
$\mathrm{P}(1)-\mathrm{N}(2)$ & $1.7190(13)$ & $1.7341(8)$ & $1.696(3)$ \\
$\mathrm{P}(1)-\mathrm{N}(3)$ & $1.7014(14)$ & $1.7786(8)$ & $1.782(3)$ \\
& & & \\
$\mathrm{N}(2)-\mathrm{P}(1)-\mathrm{N}(3)$ & $115.21(7)$ & $108.67(4)$ & $110.55(18)$ \\
$\mathrm{N}(1)-\mathrm{P}(1)-\mathrm{N}(2)$ & $90.08(6)$ & $90.56(4)$ & $91.54(17)$ \\
$\mathrm{N}(1)-\mathrm{P}(1)-\mathrm{N}(3)$ & $90.51(6)$ & $89.45(4)$ & $89.18(16)$ \\
\hline
\end{tabular}


Table S6. Selected metrical parameters for complexes 4, $\mathbf{5}^{\prime}$ and $\mathbf{6}^{\prime}$

\begin{tabular}{cccc}
\hline & $\mathbf{4}(\mathrm{M}=\mathrm{Pt})$ & $\mathbf{5}^{\prime}(\mathrm{M}=\mathrm{Pd})$ & $\mathbf{6}^{\prime}(\mathrm{M}=\mathrm{Ni})$ \\
\hline $\mathrm{P}(1)-\mathrm{N}(1)$ & $1.730(5)$ & $1.716(5)$ & $1.761(4)$ \\
$\mathrm{P}(1)-\mathrm{N}(2)$ & $1.670(5)$ & $2.002(5)$ & $1.990(4)$ \\
$\mathrm{P}(1)-\mathrm{N}(3)$ & $1.717(5)$ & $1.766(5)$ & $1.720(13)$ \\
$\mathrm{P}(1)-\mathrm{C}(1)$ & - & $1.815(6)$ & $1.834(4)$ \\
$\mathrm{M}(1)-\mathrm{C}(1)$ & $2.126(7)$ & - & - \\
$\mathrm{M}(1)-\mathrm{N}(2)$ & - & $2.002(5)$ & $1.993(3)$ \\
$\mathrm{M}(1)-\mathrm{Cl}(1)$ & $2.3559(16)$ & $2.3752(17)$ & $2.2004(12)$ \\
$\mathrm{M}(1)-\mathrm{P}(1)$ & $2.1642(14)$ & $2.1434(18)$ & $2.0511(12)$ \\
$\mathrm{M}(1)-\mathrm{P}(2)$ & $2.3409(15)$ & $2.2567(17)$ & $2.1748(12)$ \\
$\mathrm{N}(2)-\mathrm{P}(1)-\mathrm{M}(1)$ & $115.87(18)$ & $63.34(16)$ & $61.99(11)$ \\
$\mathrm{N}(2)-\mathrm{P}(1)-\mathrm{N}(3)$ & $115.5(2)$ & $166.7(2)$ & $165.76(17)$ \\
$\mathrm{N}(2)-\mathrm{P}(1)-\mathrm{N}(1)$ & $93.2(2)$ & $85.5(2)$ & $85.74(16)$ \\
$\mathrm{N}(3)-\mathrm{P}(1)-\mathrm{N}(1)$ & $91.5(2)$ & $88.0(2)$ & $88.67(17)$ \\
$\mathrm{C}(1)-\mathrm{P}(1)-\mathrm{N}(1)$ & - & $112.6(3)$ & $110.6(2)$ \\
$\mathrm{C}(1)-\mathrm{P}(1)-\mathrm{M}(1)$ & - & $126.8(2)$ & $127.39(16)$ \\
$\mathrm{P}(1)-\mathrm{M}(1)-\mathrm{P}(2)$ & $94.45(5)$ & $98.76(6)$ & $98.82(5)$ \\
$\mathrm{P}(1)-\mathrm{M}(1)-\mathrm{C}(1)$ & $86.46(19)$ & - & - \\
$\mathrm{P}(1)-\mathrm{M}(1)-\mathrm{Cl}(1)$ & $173.81(6)$ & $154.29(6)$ & $154.09(5)$ \\
$\mathrm{C}(1)-\mathrm{M}(1)-\mathrm{Cl}(1)$ & $88.61(19)$ & - & - \\
$\mathrm{P}(2)-\mathrm{M}(1)-\mathrm{Cl}(1)$ & $90.65(5)$ & $103.84(6)$ & $102.56(4)$ \\
$\tau_{4}$ for metal center & 0.06 & 0.37 & 0.34 \\
$\tau_{5}$ for $\mathrm{P}$ center & $\mathrm{N} / \mathrm{A}$ & 0.67 & 0.64 \\
\hline
\end{tabular}

${ }^{a} \tau_{4}$ is a metric for four coordinate complexes. $\tau_{4}=0$ for a perfect square planar and $\tau_{4}=1$ for a perfect tetrahedral. $\tau_{5}$ is a metric for five coordinate complexes. $\tau_{5}=0$ for a perfect square pyramid and $\tau_{5}=1$ for a perfect trigonal bipyramid. 


\section{DFT calculations.}

\section{D.1. Computational Details}

DFT calculations at the B3LYP level of theory were performed using the Orca 4.0.07. Gas-phase geometry optimizations and frequency calculations for thermochemistry were carried out using the def2-TZVP basis set all atoms without symmetry constraint. B3LYP geometries well reproduced experimental metrical parameters, obtained by X-ray crystallography (tabulated in Table S7). Effective oxidation analysis (EOS) was conducted based on the Kohn-Sham wave functions computed at B3LYP/def2-TZVP level. ${ }^{7}$ The analysis of intrinsic bond orbitals (IBOs) and the visualization of orbitals were conducted by IBOview. ${ }^{8}$ The localized orbital bonding analysis (LOBA) was performed by Multiwfn. ${ }^{9}$ All occupied orbitals were localized with Pipek-Mezey localization procedure ${ }^{10}$ and used in the LOBA analysis. For topological analysis of the electron density (QTAIM), ${ }^{11}$ an all-electron single point calculation was conducted. A relativistic approximation was introduced by applying zeroth-order regular approximation (ZORA) ${ }^{12}$ using the B3LYP density functional with the relativistically recontracted version of def2- TZVP basis set. Coordinates obtained from the above geometry optimization is used as the input. Analysis and visualization of the QTAIM results were performed with the Multiwfn program. 
D.2. Comparison of Crystallographic and Computed Structures of 4, $5^{\prime}$ and $6^{\prime}$ Table S7. Selected metrical parameters for complexes $4,5^{\prime}$ and $6^{\prime}$.

\begin{tabular}{|c|c|c|c|c|c|c|}
\hline & \multicolumn{2}{|c|}{$4(\mathrm{M}=\mathrm{Pt})$} & \multicolumn{2}{|c|}{$5^{\prime}(\mathrm{M}=\mathrm{Pd})$} & \multicolumn{2}{|c|}{$6^{\prime}(\mathrm{M}=\mathrm{Ni})$} \\
\hline & Experimental & Calculated & Experimental & Calculated & Experimental & Calculated \\
\hline $\mathrm{P}(1)-\mathrm{N}(1)$ & $1.730(5)$ & 1.728 & $1.716(5)$ & 1.718 & $1.761(4)$ & 1.715 \\
\hline $\mathrm{P}(1)-\mathrm{N}(2)$ & $1.670(5)$ & 1.704 & $2.002(5)$ & 2.024 & $1.990(4)$ & 2.006 \\
\hline $\mathrm{P}(1)-\mathrm{N}(3)$ & $1.717(5)$ & 1.728 & $1.766(5)$ & 1.800 & $1.720(13)$ & 1.759 \\
\hline $\mathrm{P}(1)-\mathrm{C}(1)$ & - & - & $1.815(6)$ & 1.827 & $1.834(4)$ & 1.827 \\
\hline $\mathrm{M}(1)-\mathrm{C}(1)$ & $2.126(7)$ & 2.115 & - & - & - & - \\
\hline $\mathrm{M}(1)-\mathrm{N}(2)$ & - & - & $2.002(5)$ & 2.240 & $1.993(3)$ & 2.186 \\
\hline $\mathrm{M}(1)-\mathrm{Cl}(1)$ & $2.3559(16)$ & 2.393 & $2.3752(17)$ & 2.402 & $2.2004(12)$ & 2.371 \\
\hline $\mathrm{M}(1)-\mathrm{P}(1)$ & $2.1642(14)$ & 2.171 & $2.1434(18)$ & 2.177 & $2.0511(12)$ & 2.145 \\
\hline $\mathrm{M}(1)-\mathrm{P}(2)$ & $2.3409(15)$ & 2.399 & $2.2567(17)$ & 2.287 & $2.1748(12)$ & 2.258 \\
\hline $\mathrm{N}(2)-\mathrm{P}(1)-\mathrm{M}(1)$ & $115.87(18)$ & 116.9 & $63.34(16)$ & 64.3 & $61.99(11)$ & 63.5 \\
\hline $\mathrm{N}(2)-\mathrm{P}(1)-\mathrm{N}(3)$ & $115.5(2)$ & 114.1 & $166.7(2)$ & 166.0 & $165.76(17)$ & 166.9 \\
\hline $\mathrm{N}(2)-\mathrm{P}(1)-\mathrm{N}(1)$ & $93.2(2)$ & 92.2 & $85.5(2)$ & 85.1 & $85.74(16)$ & 85.3 \\
\hline $\mathrm{N}(3)-\mathrm{P}(1)-\mathrm{N}(1)$ & $91.5(2)$ & 91.5 & $88.0(2)$ & 88.0 & $88.67(17)$ & 88.4 \\
\hline $\mathrm{C}(1)-\mathrm{P}(1)-\mathrm{N}(1)$ & - & - & $112.6(3)$ & 112.6 & $110.6(2)$ & 113.1 \\
\hline $\mathrm{C}(1)-\mathrm{P}(1)-\mathrm{M}(1)$ & - & - & $126.8(2)$ & 123.5 & $127.39(16)$ & 126.3 \\
\hline $\mathrm{P}(1)-\mathrm{M}(1)-\mathrm{P}(2)$ & $94.45(5)$ & 94.7 & $98.76(6)$ & 98.6 & $98.82(5)$ & 98.8 \\
\hline $\mathrm{P}(1)-\mathrm{M}(1)-\mathrm{C}(1)$ & $86.46(19)$ & 89.2 & - & - & - & - \\
\hline $\mathrm{P}(1)-\mathrm{M}(1)-\mathrm{Cl}(1)$ & $173.81(6)$ & 174.7 & $154.29(6)$ & 158.9 & $154.09(5)$ & 154.3 \\
\hline $\mathrm{C}(1)-\mathrm{M}(1)-\mathrm{Cl}(1)$ & $88.61(19)$ & 85.6 & - & - & - & - \\
\hline $\mathrm{P}(2)-\mathrm{M}(1)-\mathrm{Cl}(1)$ & $90.65(5)$ & 90.5 & $103.84(6)$ & 101.8 & $102.56(4)$ & 103.8 \\
\hline
\end{tabular}




\section{D.3. Comparison of Crystallographic and Computed Structures of 4}

Table S8. Relationships between $J_{\mathrm{Pt}-\mathrm{P}}$ values and Pt-P bond lengths in 4-cis and 4-trans.

\begin{tabular}{ccccccc}
\hline & \multicolumn{3}{c}{ 4-cis } & \multicolumn{3}{c}{ 4-trans } \\
\cline { 2 - 7 } & ${ }^{1} J_{\mathrm{Pt}-\mathrm{P}}(\mathrm{Hz})^{a}$ & $d_{\text {derived }}{ }^{b}$ & $d_{\text {calc }}$ & ${ }^{1} J_{\mathrm{Pt}-\mathrm{P}}(\mathrm{Hz})^{a}$ & $d_{\text {derived }}{ }^{b}$ & $d_{\text {calc }^{c}}$ \\
\hline $\mathrm{Pt}(1)-\mathrm{P}(1)$ & 2601 & 2.314 & 2.286 & 6700 & 2.145 & 2.171 \\
$\mathrm{Pt}(1)-\mathrm{P}(2)$ & 4048 & 2.254 & 2.265 & 1695 & 2.351 & 2.399
\end{tabular}

${ }^{a}$ Experimental values in ${ }^{31} \mathrm{P}$ NMR spectra. ${ }^{b}$ Bond distance derived from ${ }^{1} \mathrm{Jt}_{\mathrm{Pt}} \mathrm{P}$ based on the correlation in Woollins' paper. ${ }^{c}$ Bond distance calculated by DFT calculation at B3LYP/def2TZVP level.

\section{D.4. Energies and XYZ Coordinates}

Table S9. Energy of 8-P-4 and 10-P-5, and their differences in energies (Hartree/Particle) for group 10 metal complexes $(\mathrm{Pt}, \mathrm{Pd}$, and $\mathrm{Ni})$.

\begin{tabular}{|c|c|c|c|}
\hline & $\begin{array}{c}\mathrm{PtClMeiPr} 2 \mathrm{PNNNP} \\
(4,8-\mathrm{P}-4)\end{array}$ & $\begin{array}{c}\mathrm{PtCliPr}_{2} \mathrm{PNNNPMe} \\
\left(4^{\prime}, 10-\mathrm{P}-5\right)\end{array}$ & $\begin{array}{c}\text { Energy } \\
\text { Difference }\end{array}$ \\
\hline Thermal Energy, E & -2627.935335 & -2627.940321 & -0.004986 \\
\hline Thermal Enthalpy, H & -2627.317284 & -2627.321633 & -0.004349 \\
\hline \multirow[t]{2}{*}{ Thermal Free Energy, G } & -2627.418196 & -2627.421284 & -0.003088 \\
\hline & $\begin{array}{c}\mathrm{PdClMeiPr}_{2} \mathrm{PNNNP} \\
(\mathbf{5}, 8-\mathrm{P}-4)\end{array}$ & $\begin{array}{c}\mathrm{PdCliPr}_{2} \mathrm{PNNNPMe} \\
\left(5^{\prime}, 10-\mathrm{P}-5\right)\end{array}$ & $\begin{array}{c}\text { Energy } \\
\text { Difference }\end{array}$ \\
\hline Thermal Energy, E & -2636.465437 & -2636.481724 & -0.016286 \\
\hline Thermal Enthalpy, H & -2635.847719 & -2635.863005 & -0.015286 \\
\hline \multirow[t]{2}{*}{ Thermal Free Energy, G } & -2635.948877 & -2635.962377 & -0.013500 \\
\hline & $\begin{array}{c}\mathrm{NiClMeiPr}_{2} \mathrm{PNNNP} \\
(\mathbf{6}, 8-\mathrm{P}-4)\end{array}$ & $\begin{array}{c}\mathrm{NiCliPr}_{2} \mathrm{PNNNPMe} \\
\left(6^{\prime}, 10-\mathrm{P}-5\right)\end{array}$ & $\begin{array}{c}\text { Energy } \\
\text { Difference }\end{array}$ \\
\hline Thermal Energy, E & -4016.834067 & -4016.860843 & -0.026777 \\
\hline Thermal Enthalpy, H & -4016.216102 & -4016.242079 & -0.025977 \\
\hline Thermal Free Energy, G & -4016.315554 & -4016.340775 & -0.025221 \\
\hline
\end{tabular}




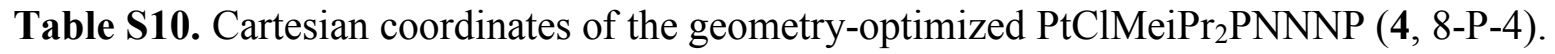

\begin{tabular}{|c|c|c|c|}
\hline Atom Type & $\mathrm{x}$ & $\mathrm{y}$ & $\mathrm{z}$ \\
\hline $\mathrm{P}$ & 19.6174 & 11.91152 & 4.57151 \\
\hline $\mathrm{P}$ & 16.75599 & 11.06654 & 3.01304 \\
\hline $\mathrm{N}$ & 20.00573 & 11.57939 & 6.19665 \\
\hline $\mathrm{C}$ & 18.44708 & 12.08838 & 8.02065 \\
\hline $\mathrm{H}$ & 18.68778 & 13.13761 & 7.9155 \\
\hline $\mathrm{N}$ & 21.18743 & 12.56317 & 4.25896 \\
\hline $\mathrm{N}$ & 19.8998 & 10.59057 & 3.49434 \\
\hline $\mathrm{C}$ & 17.52975 & 11.66428 & 8.97316 \\
\hline $\mathrm{H}$ & 17.05534 & 12.39183 & 9.61962 \\
\hline $\mathrm{C}$ & 17.20905 & 10.31462 & 9.0868 \\
\hline $\mathrm{H}$ & 16.48948 & 9.98876 & 9.82745 \\
\hline $\mathrm{C}$ & 21.27924 & 12.0691 & 6.54936 \\
\hline $\mathrm{C}$ & 23.79609 & 13.17668 & 6.88828 \\
\hline $\mathrm{H}$ & 24.77626 & 13.6146 & 7.0246 \\
\hline $\mathrm{C}$ & 15.62084 & 12.39504 & 0.77528 \\
\hline $\mathrm{H}$ & 16.00265 & 13.35676 & 1.11601 \\
\hline $\mathrm{H}$ & 15.52526 & 12.42592 & -0.31362 \\
\hline $\mathrm{H}$ & 14.62225 & 12.27857 & 1.1947 \\
\hline $\mathrm{C}$ & 21.94475 & 12.65208 & 5.46168 \\
\hline $\mathrm{C}$ & 19.05345 & 11.15712 & 7.1787 \\
\hline $\mathrm{C}$ & 17.69836 & 9.47874 & 3.14672 \\
\hline $\mathrm{C}$ & 23.19781 & 13.21467 & 5.62722 \\
\hline $\mathrm{H}$ & 23.70723 & 13.68116 & 4.79696 \\
\hline $\mathrm{C}$ & 23.13853 & 12.59214 & 7.96192 \\
\hline $\mathrm{H}$ & 23.60699 & 12.57235 & 8.93765 \\
\hline $\mathrm{C}$ & 21.87089 & 12.03137 & 7.80253 \\
\hline $\mathrm{H}$ & 21.35699 & 11.57566 & 8.6378 \\
\hline $\mathrm{C}$ & 17.81123 & 9.38752 & 8.24478 \\
\hline $\mathrm{H}$ & 17.56585 & 8.33611 & 8.32767 \\
\hline $\mathrm{C}$ & 21.03684 & 10.82007 & 2.69531 \\
\hline $\mathrm{C}$ & 16.56678 & 11.25142 & 1.15899 \\
\hline $\mathrm{H}$ & 16.13835 & 10.30295 & 0.81706 \\
\hline $\mathrm{C}$ & 23.25383 & 11.68355 & 1.25473 \\
\hline $\mathrm{H}$ & 24.11101 & 12.02087 & 0.68706 \\
\hline $\mathrm{C}$ & 19.08872 & 9.42931 & 3.38275 \\
\hline $\mathrm{C}$ & 22.8323 & 12.4139 & 2.3666 \\
\hline $\mathrm{H}$ & 23.34365 & 13.3224 & 2.65041 \\
\hline $\mathrm{C}$ & 19.73143 & 8.19357 & 3.51622 \\
\hline $\mathrm{H}$ & 20.79383 & 8.18477 & 3.72247 \\
\hline $\mathrm{C}$ & 17.02726 & 8.25596 & 3.01423 \\
\hline $\mathrm{H}$ & 15.96911 & 8.25766 & 2.80727 \\
\hline $\mathrm{C}$ & 14.02593 & 9.93327 & 2.87411 \\
\hline $\mathrm{H}$ & 14.14087 & 8.86255 & 3.04809 \\
\hline
\end{tabular}




\begin{tabular}{|c|c|c|c|}
\hline $\mathrm{H}$ & 13.0151 & 10.1936 & 3.19939 \\
\hline $\mathrm{H}$ & 14.07282 & 10.1103 & 1.79993 \\
\hline $\mathrm{C}$ & 18.73639 & 9.80617 & 7.29403 \\
\hline $\mathrm{H}$ & 19.21667 & 9.08967 & 6.63994 \\
\hline $\mathrm{C}$ & 21.45002 & 10.10113 & 1.58085 \\
\hline $\mathrm{H}$ & 20.9158 & 9.216 & 1.26539 \\
\hline $\mathrm{C}$ & 17.92936 & 11.43297 & 0.48252 \\
\hline $\mathrm{H}$ & 18.60299 & 10.59695 & 0.66908 \\
\hline $\mathrm{H}$ & 17.79076 & 11.50942 & -0.5989 \\
\hline $\mathrm{H}$ & 18.41835 & 12.34826 & 0.82086 \\
\hline $\mathrm{C}$ & 15.02892 & 10.77501 & 3.67589 \\
\hline $\mathrm{H}$ & 14.67772 & 11.81172 & 3.65677 \\
\hline $\mathrm{C}$ & 21.73952 & 11.9706 & 3.08944 \\
\hline $\mathrm{C}$ & 19.03755 & 7.00268 & 3.38292 \\
\hline $\mathrm{H}$ & 19.55915 & 6.05939 & 3.48677 \\
\hline $\mathrm{C}$ & 15.06892 & 10.34726 & 5.14781 \\
\hline $\mathrm{H}$ & 15.69639 & 11.01151 & 5.74235 \\
\hline $\mathrm{H}$ & 14.0588 & 10.38243 & 5.56379 \\
\hline $\mathrm{H}$ & 15.44125 & 9.32743 & 5.26664 \\
\hline $\mathrm{C}$ & 22.56771 & 10.53956 & 0.87104 \\
\hline $\mathrm{H}$ & 22.89088 & 9.98203 & 0.00102 \\
\hline $\mathrm{C}$ & 17.67375 & 7.03352 & 3.12249 \\
\hline $\mathrm{H}$ & 17.11329 & 6.11438 & 3.00754 \\
\hline $\mathrm{Pt}$ & 17.72664 & 12.91197 & 4.19906 \\
\hline $\mathrm{Cl}$ & 15.73788 & 14.20033 & 3.86317 \\
\hline $\mathrm{C}$ & 18.45751 & 14.61198 & 5.22332 \\
\hline $\mathrm{H}$ & 19.44297 & 14.51572 & 5.68105 \\
\hline $\mathrm{H}$ & 18.49016 & 15.4252 & 4.49705 \\
\hline $\mathrm{H}$ & 17.72546 & 14.86368 & 5.99122 \\
\hline
\end{tabular}


Table S11. Cartesian coordinates of the geometry-optimized $\left.\mathrm{PtCliPr}_{2} \mathrm{PNNNPMe}^{\prime}{ }^{\prime}, 10-\mathrm{P}-5\right)$.

\begin{tabular}{|c|c|c|c|}
\hline Atom Type & $\mathrm{x}$ & $\mathrm{y}$ & $\mathrm{z}$ \\
\hline $\mathrm{Pt}$ & 7.73642 & 3.47172 & 8.91436 \\
\hline $\mathrm{Cl}$ & 6.42444 & 2.98657 & 6.96237 \\
\hline $\mathrm{P}$ & 8.25653 & 3.97455 & 10.96311 \\
\hline $\mathrm{P}$ & 9.7594 & 2.67086 & 8.26619 \\
\hline $\mathrm{N}$ & 7.45784 & 3.07899 & 12.1799 \\
\hline $\mathrm{N}$ & 6.16583 & 4.28036 & 10.21102 \\
\hline $\mathrm{N}$ & 9.79043 & 3.26569 & 11.44021 \\
\hline $\mathrm{C}$ & 6.13693 & 2.62883 & 11.94321 \\
\hline $\mathrm{C}$ & 5.52924 & 1.56163 & 12.60347 \\
\hline $\mathrm{H}$ & 6.06343 & 1.00234 & 13.35481 \\
\hline $\mathrm{C}$ & 4.2285 & 1.19381 & 12.27712 \\
\hline $\mathrm{H}$ & 3.76667 & 0.36685 & 12.80181 \\
\hline $\mathrm{C}$ & 3.5321 & 1.86841 & 11.28094 \\
\hline $\mathrm{H}$ & 2.52141 & 1.57596 & 11.0261 \\
\hline $\mathrm{C}$ & 4.14926 & 2.90041 & 10.58768 \\
\hline $\mathrm{H}$ & 3.63881 & 3.40664 & 9.7791 \\
\hline $\mathrm{C}$ & 5.4482 & 3.2908 & 10.90723 \\
\hline $\mathrm{C}$ & 8.36382 & 2.59122 & 13.14676 \\
\hline $\mathrm{C}$ & 9.70393 & 2.67523 & 12.7086 \\
\hline $\mathrm{C}$ & 10.72125 & 2.15612 & 13.50093 \\
\hline $\mathrm{H}$ & 11.74486 & 2.16191 & 13.1561 \\
\hline $\mathrm{C}$ & 10.41186 & 1.61849 & 14.75087 \\
\hline $\mathrm{H}$ & 11.20822 & 1.21748 & 15.36486 \\
\hline $\mathrm{C}$ & 9.10567 & 1.61684 & 15.21559 \\
\hline $\mathrm{H}$ & 8.87675 & 1.23322 & 16.20127 \\
\hline $\mathrm{C}$ & 8.0734 & 2.10526 & 14.41342 \\
\hline $\mathrm{H}$ & 7.05947 & 2.11229 & 14.78353 \\
\hline $\mathrm{C}$ & 5.57454 & 5.5368 & 9.97032 \\
\hline $\mathrm{C}$ & 4.68964 & 6.10796 & 10.90138 \\
\hline $\mathrm{H}$ & 4.41558 & 5.55102 & 11.78784 \\
\hline $\mathrm{C}$ & 4.16261 & 7.37469 & 10.69453 \\
\hline $\mathrm{H}$ & 3.4804 & 7.78956 & 11.4273 \\
\hline $\mathrm{C}$ & 4.50657 & 8.11302 & 9.56569 \\
\hline $\mathrm{H}$ & 4.09273 & 9.10083 & 9.40763 \\
\hline $\mathrm{C}$ & 5.38341 & 7.55704 & 8.64184 \\
\hline $\mathrm{H}$ & 5.65317 & 8.10891 & 7.74927 \\
\hline $\mathrm{C}$ & 5.90801 & 6.28457 & 8.83479 \\
\hline $\mathrm{H}$ & 6.55925 & 5.84659 & 8.09034 \\
\hline $\mathrm{C}$ & 10.985 & 3.55694 & 10.74512 \\
\hline $\mathrm{C}$ & 11.12774 & 3.32497 & 9.35049 \\
\hline $\mathrm{C}$ & 12.3656 & 3.63787 & 8.77431 \\
\hline $\mathrm{H}$ & 12.52365 & 3.46916 & 7.72073 \\
\hline $\mathrm{C}$ & 13.42441 & 4.16481 & 9.50054 \\
\hline
\end{tabular}




\begin{tabular}{cclc}
$\mathrm{H}$ & 14.3611 & 4.38417 & 9.00449 \\
$\mathrm{C}$ & 13.2592 & 4.41965 & 10.85309 \\
$\mathrm{H}$ & 14.06044 & 4.85654 & 11.43614 \\
$\mathrm{C}$ & 12.04894 & 4.125 & 11.4576 \\
$\mathrm{H}$ & 11.90592 & 4.34698 & 12.50573 \\
$\mathrm{C}$ & 10.28129 & 3.10202 & 6.52477 \\
$\mathrm{H}$ & 11.33982 & 2.84932 & 6.443 \\
$\mathrm{C}$ & 10.10947 & 4.60528 & 6.26966 \\
$\mathrm{H}$ & 9.05344 & 4.87658 & 6.28145 \\
$\mathrm{H}$ & 10.51115 & 4.85554 & 5.2846 \\
$\mathrm{H}$ & 10.6291 & 5.21819 & 7.00787 \\
$\mathrm{C}$ & 9.53782 & 2.27729 & 5.46377 \\
$\mathrm{H}$ & 8.46016 & 2.42037 & 5.5275 \\
$\mathrm{H}$ & 9.75275 & 1.21111 & 5.54045 \\
$\mathrm{H}$ & 9.87117 & 2.60553 & 4.47563 \\
$\mathrm{C}$ & 9.8819 & 0.81056 & 8.36502 \\
$\mathrm{H}$ & 9.1511 & 0.49434 & 7.61462 \\
$\mathrm{C}$ & 11.26154 & 0.26108 & 7.98697 \\
$\mathrm{H}$ & 11.58471 & 0.57344 & 6.99328 \\
$\mathrm{H}$ & 11.22938 & -0.83136 & 7.99064 \\
$\mathrm{H}$ & 12.02362 & 0.56926 & 8.70537 \\
$\mathrm{C}$ & 9.42219 & 0.26403 & 9.72049 \\
$\mathrm{H}$ & 10.10064 & 0.55295 & 10.52487 \\
$\mathrm{H}$ & 9.40523 & -0.8279 & 9.68079 \\
$\mathrm{H}$ & 8.41958 & 0.60648 & 9.97521 \\
$\mathrm{C}$ & 8.49087 & 5.69553 & 11.51124 \\
$\mathrm{H}$ & 9.37697 & 6.09796 & 11.01807 \\
$\mathrm{H}$ & 8.6548 & 5.6988 & 12.59056 \\
$\mathrm{H}$ & 7.63071 & 6.31294 & 11.26596 \\
\hline & & & \\
\hline
\end{tabular}


Table S12. Cartesian coordinates of the geometry-optimized $\operatorname{PdClMeiPr}_{2} \mathrm{PNNNP}(5,8-\mathrm{P}-4)$.

\begin{tabular}{|c|c|c|c|}
\hline Atom Type & $\mathrm{x}$ & $\mathrm{y}$ & $\mathrm{z}$ \\
\hline $\mathrm{P}$ & 19.61844 & 11.92979 & 4.5801 \\
\hline $\mathrm{P}$ & 16.74874 & 11.05319 & 2.99687 \\
\hline $\mathrm{N}$ & 20.0055 & 11.57931 & 6.20353 \\
\hline $\mathrm{C}$ & 18.46517 & 12.11689 & 8.03465 \\
\hline $\mathrm{H}$ & 18.7381 & 13.15941 & 7.94147 \\
\hline $\mathrm{N}$ & 21.19666 & 12.57012 & 4.27308 \\
\hline $\mathrm{N}$ & 19.89322 & 10.61693 & 3.48122 \\
\hline $\mathrm{C}$ & 17.53554 & 11.71157 & 8.98345 \\
\hline $\mathrm{H}$ & 17.08356 & 12.44686 & 9.63713 \\
\hline $\mathrm{C}$ & 17.17424 & 10.37124 & 9.08421 \\
\hline $\mathrm{H}$ & 16.44535 & 10.06024 & 9.82206 \\
\hline $\mathrm{C}$ & 21.28834 & 12.04399 & 6.55767 \\
\hline $\mathrm{C}$ & 23.82161 & 13.10982 & 6.9028 \\
\hline $\mathrm{H}$ & 24.80828 & 13.53207 & 7.04157 \\
\hline $\mathrm{C}$ & 15.57142 & 12.35836 & 0.7708 \\
\hline $\mathrm{H}$ & 15.91608 & 13.32412 & 1.13922 \\
\hline $\mathrm{H}$ & 15.48893 & 12.40978 & -0.31842 \\
\hline $\mathrm{H}$ & 14.57151 & 12.19946 & 1.17302 \\
\hline $\mathrm{C}$ & 21.95776 & 12.63247 & 5.47567 \\
\hline $\mathrm{C}$ & 19.04292 & 11.17595 & 7.18305 \\
\hline $\mathrm{C}$ & 17.70458 & 9.47359 & 3.1311 \\
\hline $\mathrm{C}$ & 23.2196 & 13.17454 & 5.64468 \\
\hline $\mathrm{H}$ & 23.73326 & 13.64493 & 4.81933 \\
\hline $\mathrm{C}$ & 23.16018 & 12.51876 & 7.97052 \\
\hline $\mathrm{H}$ & 23.63192 & 12.47828 & 8.944 \\
\hline $\mathrm{C}$ & 21.88443 & 11.97789 & 7.8075 \\
\hline $\mathrm{H}$ & 21.36701 & 11.51686 & 8.6377 \\
\hline $\mathrm{C}$ & 17.74765 & 9.43462 & 8.23266 \\
\hline $\mathrm{H}$ & 17.47019 & 8.39048 & 8.30517 \\
\hline $\mathrm{C}$ & 21.03433 & 10.846 & 2.68892 \\
\hline $\mathrm{C}$ & 16.54737 & 11.23656 & 1.14359 \\
\hline $\mathrm{H}$ & 16.14352 & 10.27923 & 0.79611 \\
\hline $\mathrm{C}$ & 23.25596 & 11.71291 & 1.25713 \\
\hline $\mathrm{H}$ & 24.11411 & 12.05239 & 0.69228 \\
\hline $\mathrm{C}$ & 19.09469 & 9.44449 & 3.37496 \\
\hline $\mathrm{C}$ & 22.83805 & 12.43389 & 2.37651 \\
\hline $\mathrm{H}$ & 23.35377 & 13.3371 & 2.66925 \\
\hline $\mathrm{C}$ & 19.75237 & 8.21814 & 3.52196 \\
\hline $\mathrm{H}$ & 20.81388 & 8.22427 & 3.73297 \\
\hline $\mathrm{C}$ & 17.05036 & 8.24092 & 3.00379 \\
\hline $\mathrm{H}$ & 15.99337 & 8.22785 & 2.79044 \\
\hline $\mathrm{C}$ & 14.03218 & 9.88729 & 2.89635 \\
\hline $\mathrm{H}$ & 14.15797 & 8.82343 & 3.10264 \\
\hline
\end{tabular}




\begin{tabular}{|c|c|c|c|}
\hline $\mathrm{H}$ & 13.01881 & 10.14786 & 3.21345 \\
\hline $\mathrm{H}$ & 14.07837 & 10.03243 & 1.81719 \\
\hline $\mathrm{C}$ & 18.68454 & 9.8341 & 7.28529 \\
\hline $\mathrm{H}$ & 19.14255 & 9.10975 & 6.62388 \\
\hline $\mathrm{C}$ & 21.44535 & 10.13521 & 1.56826 \\
\hline $\mathrm{H}$ & 20.90725 & 9.25527 & 1.24482 \\
\hline $\mathrm{C}$ & 17.90411 & 11.45833 & 0.46723 \\
\hline $\mathrm{H}$ & 18.60465 & 10.64564 & 0.65823 \\
\hline $\mathrm{H}$ & 17.76475 & 11.52594 & -0.61467 \\
\hline $\mathrm{H}$ & 18.3629 & 12.3909 & 0.80127 \\
\hline $\mathrm{C}$ & 15.02859 & 10.75985 & 3.67258 \\
\hline $\mathrm{H}$ & 14.66898 & 11.7935 & 3.63264 \\
\hline $\mathrm{C}$ & 21.74361 & 11.98799 & 3.09515 \\
\hline $\mathrm{C}$ & 19.07479 & 7.01719 & 3.3941 \\
\hline $\mathrm{H}$ & 19.60826 & 6.08171 & 3.50786 \\
\hline $\mathrm{C}$ & 15.08194 & 10.36647 & 5.15324 \\
\hline $\mathrm{H}$ & 15.69753 & 11.05657 & 5.73084 \\
\hline $\mathrm{H}$ & 14.07315 & 10.39157 & 5.57311 \\
\hline $\mathrm{H}$ & 15.47477 & 9.35698 & 5.29352 \\
\hline $\mathrm{C}$ & 22.56499 & 10.57546 & 0.86273 \\
\hline $\mathrm{H}$ & 22.88569 & 10.025 & -0.01267 \\
\hline $\mathrm{C}$ & 17.71221 & 7.02794 & 3.1255 \\
\hline $\mathrm{H}$ & 17.16482 & 6.10051 & 3.01404 \\
\hline $\mathrm{Pd}$ & 17.71266 & 12.93091 & 4.19191 \\
\hline $\mathrm{Cl}$ & 15.73268 & 14.21951 & 3.85395 \\
\hline $\mathrm{C}$ & 18.48129 & 14.59931 & 5.20855 \\
\hline $\mathrm{H}$ & 19.46792 & 14.48997 & 5.6577 \\
\hline $\mathrm{H}$ & 18.50365 & 15.39116 & 4.46131 \\
\hline $\mathrm{H}$ & 17.73924 & 14.83235 & 5.96995 \\
\hline
\end{tabular}




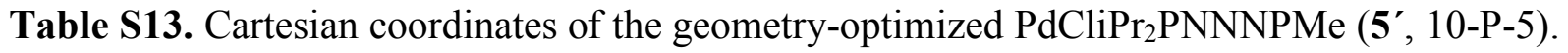

\begin{tabular}{|c|c|c|c|}
\hline Atom Type & $\mathrm{x}$ & $\mathrm{y}$ & $\mathrm{z}$ \\
\hline $\mathrm{Pd}$ & 7.69149 & 3.39745 & 8.88171 \\
\hline $\mathrm{Cl}$ & 6.39446 & 2.85916 & 6.93346 \\
\hline $\mathrm{P}$ & 8.12784 & 3.95106 & 10.94147 \\
\hline $\mathrm{P}$ & 9.7702 & 2.66087 & 8.27792 \\
\hline $\mathrm{N}$ & 7.41806 & 3.00133 & 12.18534 \\
\hline $\mathrm{N}$ & 6.22328 & 4.26522 & 10.33424 \\
\hline $\mathrm{N}$ & 9.72061 & 3.25163 & 11.40506 \\
\hline $\mathrm{C}$ & 6.07984 & 2.60372 & 12.0041 \\
\hline $\mathrm{C}$ & 5.42049 & 1.56678 & 12.66135 \\
\hline $\mathrm{H}$ & 5.92548 & 0.96767 & 13.40161 \\
\hline $\mathrm{C}$ & 4.0975 & 1.28293 & 12.33638 \\
\hline $\mathrm{H}$ & 3.58902 & 0.47942 & 12.85416 \\
\hline $\mathrm{C}$ & 3.43481 & 2.00808 & 11.35193 \\
\hline $\mathrm{H}$ & 2.40742 & 1.77575 & 11.10254 \\
\hline $\mathrm{C}$ & 4.10175 & 3.0127 & 10.66276 \\
\hline $\mathrm{H}$ & 3.61438 & 3.5572 & 9.86512 \\
\hline $\mathrm{C}$ & 5.42034 & 3.31434 & 10.98672 \\
\hline $\mathrm{C}$ & 8.34763 & 2.48239 & 13.10738 \\
\hline $\mathrm{C}$ & 9.67274 & 2.6096 & 12.64033 \\
\hline $\mathrm{C}$ & 10.7173 & 2.073 & 13.38746 \\
\hline $\mathrm{H}$ & 11.73277 & 2.11767 & 13.02087 \\
\hline $\mathrm{C}$ & 10.44692 & 1.47623 & 14.61897 \\
\hline $\mathrm{H}$ & 11.26437 & 1.06402 & 15.19705 \\
\hline $\mathrm{C}$ & 9.15202 & 1.42813 & 15.11244 \\
\hline $\mathrm{H}$ & 8.95245 & 0.99591 & 16.08428 \\
\hline $\mathrm{C}$ & 8.09325 & 1.93319 & 14.35575 \\
\hline $\mathrm{H}$ & 7.0902 & 1.90946 & 14.75286 \\
\hline $\mathrm{C}$ & 5.66234 & 5.53638 & 10.01988 \\
\hline $\mathrm{C}$ & 4.88962 & 6.21504 & 10.97342 \\
\hline $\mathrm{H}$ & 4.68635 & 5.74433 & 11.92689 \\
\hline $\mathrm{C}$ & 4.38429 & 7.47798 & 10.70185 \\
\hline $\mathrm{H}$ & 3.78781 & 7.98493 & 11.45089 \\
\hline $\mathrm{C}$ & 4.64479 & 8.09516 & 9.48086 \\
\hline $\mathrm{H}$ & 4.24993 & 9.08131 & 9.2712 \\
\hline $\mathrm{C}$ & 5.40665 & 7.42594 & 8.53201 \\
\hline $\mathrm{H}$ & 5.60216 & 7.88397 & 7.57012 \\
\hline $\mathrm{C}$ & 5.9064 & 6.15362 & 8.79298 \\
\hline $\mathrm{H}$ & 6.45485 & 5.61661 & 8.03013 \\
\hline $\mathrm{C}$ & 10.91177 & 3.61097 & 10.74211 \\
\hline $\mathrm{C}$ & 11.09732 & 3.38516 & 9.35379 \\
\hline $\mathrm{C}$ & 12.32238 & 3.76938 & 8.79371 \\
\hline $\mathrm{H}$ & 12.5068 & 3.60651 & 7.7429 \\
\hline $\mathrm{C}$ & 13.33395 & 4.359 & 9.53844 \\
\hline
\end{tabular}




\begin{tabular}{|c|c|c|c|}
\hline $\mathrm{H}$ & 14.26558 & 4.63308 & 9.06015 \\
\hline $\mathrm{C}$ & 13.13089 & 4.60112 & 10.88931 \\
\hline $\mathrm{H}$ & 13.89732 & 5.0807 & 11.48566 \\
\hline $\mathrm{C}$ & 11.92969 & 4.23726 & 11.47389 \\
\hline $\mathrm{H}$ & 11.7591 & 4.44125 & 12.52208 \\
\hline $\mathrm{C}$ & 10.27666 & 3.09463 & 6.53293 \\
\hline $\mathrm{H}$ & 11.34723 & 2.89295 & 6.45726 \\
\hline $\mathrm{C}$ & 10.03266 & 4.58523 & 6.26231 \\
\hline $\mathrm{H}$ & 8.96364 & 4.80162 & 6.26335 \\
\hline $\mathrm{H}$ & 10.42807 & 4.84608 & 5.27747 \\
\hline $\mathrm{H}$ & 10.51626 & 5.23047 & 6.99756 \\
\hline $\mathrm{C}$ & 9.57232 & 2.22572 & 5.48125 \\
\hline $\mathrm{H}$ & 8.48829 & 2.30952 & 5.55501 \\
\hline $\mathrm{H}$ & 9.84827 & 1.17364 & 5.55918 \\
\hline $\mathrm{H}$ & 9.87748 & 2.5686 & 4.48902 \\
\hline $\mathrm{C}$ & 9.95931 & 0.80638 & 8.39362 \\
\hline $\mathrm{H}$ & 9.26712 & 0.46026 & 7.62042 \\
\hline $\mathrm{C}$ & 11.37062 & 0.30995 & 8.06269 \\
\hline $\mathrm{H}$ & 11.71427 & 0.63783 & 7.08081 \\
\hline $\mathrm{H}$ & 11.38036 & -0.78299 & 8.06411 \\
\hline $\mathrm{H}$ & 12.09506 & 0.64697 & 8.80653 \\
\hline $\mathrm{C}$ & 9.47234 & 0.24588 & 9.73326 \\
\hline $\mathrm{H}$ & 10.11092 & 0.55692 & 10.56111 \\
\hline $\mathrm{H}$ & 9.49127 & -0.84606 & 9.69367 \\
\hline $\mathrm{H}$ & 8.45024 & 0.5549 & 9.95253 \\
\hline $\mathrm{C}$ & 8.46915 & 5.66467 & 11.47566 \\
\hline $\mathrm{H}$ & 9.39749 & 5.99708 & 11.01143 \\
\hline $\mathrm{H}$ & 8.60886 & 5.65492 & 12.55875 \\
\hline $\mathrm{H}$ & 7.66751 & 6.34908 & 11.216 \\
\hline
\end{tabular}


Table S14. Cartesian coordinates of the geometry-optimized NiClMeiPr ${ }_{2} \mathrm{PNNNP}(6,8-\mathrm{P}-4)$.

\begin{tabular}{|c|c|c|c|}
\hline Atom Type & $\mathrm{x}$ & $\mathrm{y}$ & $\mathrm{z}$ \\
\hline $\mathrm{P}$ & 19.58116 & 11.92493 & 4.53479 \\
\hline $\mathrm{P}$ & 16.78566 & 11.09197 & 3.05906 \\
\hline $\mathrm{N}$ & 19.96212 & 11.56311 & 6.16056 \\
\hline $\mathrm{C}$ & 18.38448 & 12.06913 & 7.96971 \\
\hline $\mathrm{H}$ & 18.62841 & 13.11841 & 7.87228 \\
\hline $\mathrm{N}$ & 21.16174 & 12.57752 & 4.24372 \\
\hline $\mathrm{N}$ & 19.89442 & 10.60988 & 3.44265 \\
\hline $\mathrm{C}$ & 17.459 & 11.64296 & 8.91373 \\
\hline $\mathrm{H}$ & 16.97911 & 12.36912 & 9.55768 \\
\hline $\mathrm{C}$ & 17.13977 & 10.29275 & 9.02384 \\
\hline $\mathrm{H}$ & 16.41513 & 9.96514 & 9.75869 \\
\hline $\mathrm{C}$ & 21.22716 & 12.06011 & 6.53235 \\
\hline $\mathrm{C}$ & 23.72905 & 13.18815 & 6.91505 \\
\hline $\mathrm{H}$ & 24.70262 & 13.63503 & 7.06836 \\
\hline $\mathrm{C}$ & 15.56028 & 12.36472 & 0.81162 \\
\hline $\mathrm{H}$ & 15.90335 & 13.34742 & 1.13133 \\
\hline $\mathrm{H}$ & 15.45197 & 12.36874 & -0.27677 \\
\hline $\mathrm{H}$ & 14.57219 & 12.21177 & 1.24394 \\
\hline $\mathrm{C}$ & 21.89975 & 12.66258 & 5.4595 \\
\hline $\mathrm{C}$ & 18.99944 & 11.13966 & 7.13107 \\
\hline $\mathrm{C}$ & 17.70263 & 9.48629 & 3.15561 \\
\hline $\mathrm{C}$ & 23.1449 & 13.23579 & 5.64762 \\
\hline $\mathrm{H}$ & 23.65899 & 13.71857 & 4.82972 \\
\hline $\mathrm{C}$ & 23.06559 & 12.58263 & 7.97311 \\
\hline $\mathrm{H}$ & 23.52266 & 12.55522 & 8.95404 \\
\hline $\mathrm{C}$ & 21.80569 & 12.01113 & 7.79143 \\
\hline $\mathrm{H}$ & 21.28706 & 11.53943 & 8.61483 \\
\hline $\mathrm{C}$ & 17.75064 & 9.36726 & 8.18615 \\
\hline $\mathrm{H}$ & 17.50662 & 8.31539 & 8.2667 \\
\hline $\mathrm{C}$ & 21.0489 & 10.83723 & 2.67282 \\
\hline $\mathrm{C}$ & 16.55817 & 11.26987 & 1.20382 \\
\hline $\mathrm{H}$ & 16.16869 & 10.29979 & 0.87623 \\
\hline $\mathrm{C}$ & 23.29301 & 11.71163 & 1.28151 \\
\hline $\mathrm{H}$ & 24.16124 & 12.05326 & 0.73357 \\
\hline $\mathrm{C}$ & 19.0997 & 9.43884 & 3.34395 \\
\hline $\mathrm{C}$ & 22.84715 & 12.4358 & 2.38799 \\
\hline $\mathrm{H}$ & 23.35108 & 13.34388 & 2.68585 \\
\hline $\mathrm{C}$ & 19.75069 & 8.20495 & 3.45231 \\
\hline $\mathrm{H}$ & 20.81894 & 8.19586 & 3.62477 \\
\hline $\mathrm{C}$ & 17.02808 & 8.26389 & 3.04316 \\
\hline $\mathrm{H}$ & 15.96378 & 8.26415 & 2.87172 \\
\hline $\mathrm{C}$ & 14.05861 & 9.9435 & 3.01152 \\
\hline $\mathrm{H}$ & 14.16105 & 8.88558 & 3.25686 \\
\hline
\end{tabular}




\begin{tabular}{|c|c|c|c|}
\hline $\mathrm{H}$ & 13.05023 & 10.23631 & 3.31613 \\
\hline $\mathrm{H}$ & 14.10992 & 10.04816 & 1.92795 \\
\hline $\mathrm{C}$ & 18.68248 & 9.78793 & 7.24303 \\
\hline $\mathrm{H}$ & 19.16998 & 9.07311 & 6.59251 \\
\hline $\mathrm{C}$ & 21.48561 & 10.12514 & 1.56224 \\
\hline $\mathrm{H}$ & 20.95848 & 9.2417 & 1.23055 \\
\hline $\mathrm{C}$ & 17.90542 & 11.50206 & 0.51135 \\
\hline $\mathrm{H}$ & 18.62405 & 10.70855 & 0.7147 \\
\hline $\mathrm{H}$ & 17.7565 & 11.54241 & -0.57065 \\
\hline $\mathrm{H}$ & 18.34749 & 12.45133 & 0.81995 \\
\hline $\mathrm{C}$ & 15.07113 & 10.82208 & 3.76072 \\
\hline $\mathrm{H}$ & 14.7183 & 11.85608 & 3.70839 \\
\hline $\mathrm{C}$ & 21.74064 & 11.98708 & 3.08658 \\
\hline $\mathrm{C}$ & 19.05318 & 7.01388 & 3.33892 \\
\hline $\mathrm{H}$ & 19.57931 & 6.07098 & 3.42183 \\
\hline $\mathrm{C}$ & 15.14221 & 10.45123 & 5.24608 \\
\hline $\mathrm{H}$ & 15.75466 & 11.15732 & 5.80719 \\
\hline $\mathrm{H}$ & 14.1371 & 10.4713 & 5.67529 \\
\hline $\mathrm{H}$ & 15.548 & 9.44884 & 5.39887 \\
\hline $\mathrm{C}$ & 22.61657 & 10.56881 & 0.8774 \\
\hline $\mathrm{H}$ & 22.95802 & 10.01676 & 0.01091 \\
\hline $\mathrm{C}$ & 17.68081 & 7.04248 & 3.12636 \\
\hline $\mathrm{H}$ & 17.11907 & 6.12219 & 3.02816 \\
\hline $\mathrm{Ni}$ & 17.76387 & 12.86809 & 4.15458 \\
\hline $\mathrm{Cl}$ & 15.94721 & 14.1249 & 3.81838 \\
\hline $\mathrm{C}$ & 18.60017 & 14.41229 & 5.07285 \\
\hline $\mathrm{H}$ & 19.57288 & 14.32024 & 5.55774 \\
\hline $\mathrm{H}$ & 18.66599 & 15.16963 & 4.29152 \\
\hline $\mathrm{H}$ & 17.8522 & 14.71146 & 5.8057 \\
\hline
\end{tabular}


Table S15. Cartesian coordinates of the geometry-optimized NiCliPr ${ }_{2} \mathrm{PNNNPMe}\left(6^{\prime}, 10-\mathrm{P}-5\right)$.

\begin{tabular}{|c|c|c|c|}
\hline Atom Type & $\mathrm{x}$ & $\mathrm{y}$ & $\mathrm{z}$ \\
\hline $\mathrm{Ni}$ & 7.69582 & 3.39351 & 9.04586 \\
\hline $\mathrm{Cl}$ & 6.4452 & 2.65006 & 7.36988 \\
\hline $\mathrm{P}$ & 8.20769 & 3.94879 & 10.97468 \\
\hline $\mathrm{P}$ & 9.68157 & 2.71826 & 8.35794 \\
\hline $\mathrm{N}$ & 7.4628 & 3.01769 & 12.21491 \\
\hline $\mathrm{N}$ & 6.29796 & 4.19436 & 10.26283 \\
\hline $\mathrm{N}$ & 9.77979 & 3.26328 & 11.47552 \\
\hline $\mathrm{C}$ & 6.14033 & 2.58508 & 11.99262 \\
\hline $\mathrm{C}$ & 5.4806 & 1.556 & 12.662 \\
\hline $\mathrm{H}$ & 5.97152 & 0.99587 & 13.44101 \\
\hline $\mathrm{C}$ & 4.17725 & 1.22768 & 12.301 \\
\hline $\mathrm{H}$ & 3.67019 & 0.4301 & 12.82925 \\
\hline $\mathrm{C}$ & 3.53412 & 1.90013 & 11.26822 \\
\hline $\mathrm{H}$ & 2.52295 & 1.6329 & 10.98939 \\
\hline $\mathrm{C}$ & 4.20309 & 2.89672 & 10.56884 \\
\hline $\mathrm{H}$ & 3.73606 & 3.39737 & 9.73168 \\
\hline $\mathrm{C}$ & 5.49998 & 3.24545 & 10.92902 \\
\hline $\mathrm{C}$ & 8.37621 & 2.52751 & 13.16806 \\
\hline $\mathrm{C}$ & 9.71192 & 2.64777 & 12.72569 \\
\hline $\mathrm{C}$ & 10.74136 & 2.12844 & 13.50462 \\
\hline $\mathrm{H}$ & 11.76342 & 2.16079 & 13.15679 \\
\hline $\mathrm{C}$ & 10.44684 & 1.55763 & 14.74312 \\
\hline $\mathrm{H}$ & 11.25304 & 1.15831 & 15.34539 \\
\hline $\mathrm{C}$ & 9.14278 & 1.51947 & 15.21207 \\
\hline $\mathrm{H}$ & 8.9246 & 1.10965 & 16.18955 \\
\hline $\mathrm{C}$ & 8.09873 & 2.00556 & 14.4234 \\
\hline $\mathrm{H}$ & 7.0876 & 1.98669 & 14.79955 \\
\hline $\mathrm{C}$ & 5.73745 & 5.44762 & 9.90696 \\
\hline $\mathrm{C}$ & 4.85275 & 6.10578 & 10.77519 \\
\hline $\mathrm{H}$ & 4.55453 & 5.62376 & 11.69709 \\
\hline $\mathrm{C}$ & 4.35896 & 7.36301 & 10.45982 \\
\hline $\mathrm{H}$ & 3.67441 & 7.85102 & 11.14328 \\
\hline $\mathrm{C}$ & 4.74132 & 8.00024 & 9.28197 \\
\hline $\mathrm{H}$ & 4.35435 & 8.98175 & 9.03933 \\
\hline $\mathrm{C}$ & 5.6171 & 7.35462 & 8.41878 \\
\hline $\mathrm{H}$ & 5.9126 & 7.82809 & 7.49034 \\
\hline $\mathrm{C}$ & 6.1056 & 6.08785 & 8.7213 \\
\hline $\mathrm{H}$ & 6.74608 & 5.57023 & 8.02024 \\
\hline $\mathrm{C}$ & 10.96484 & 3.57984 & 10.78534 \\
\hline $\mathrm{C}$ & 11.08873 & 3.36616 & 9.38801 \\
\hline $\mathrm{C}$ & 12.31259 & 3.69634 & 8.79284 \\
\hline $\mathrm{H}$ & 12.45585 & 3.53743 & 7.73515 \\
\hline $\mathrm{C}$ & 13.37694 & 4.22598 & 9.5091 \\
\hline
\end{tabular}




\begin{tabular}{cccc}
$\mathrm{H}$ & 14.30463 & 4.45908 & 9.0025 \\
$\mathrm{C}$ & 13.23008 & 4.46397 & 10.86774 \\
$\mathrm{H}$ & 14.03741 & 4.90107 & 11.44229 \\
$\mathrm{C}$ & 12.03331 & 4.15103 & 11.48984 \\
$\mathrm{H}$ & 11.90556 & 4.35856 & 12.54295 \\
$\mathrm{C}$ & 10.12106 & 3.24245 & 6.61426 \\
$\mathrm{H}$ & 11.18841 & 3.05154 & 6.49343 \\
$\mathrm{C}$ & 9.87079 & 4.74573 & 6.43824 \\
$\mathrm{H}$ & 8.80271 & 4.9626 & 6.48293 \\
$\mathrm{H}$ & 10.236 & 5.06724 & 5.45965 \\
$\mathrm{H}$ & 10.37645 & 5.3466 & 7.19604 \\
$\mathrm{C}$ & 9.39636 & 2.43521 & 5.52891 \\
$\mathrm{H}$ & 8.31523 & 2.52434 & 5.61741 \\
$\mathrm{H}$ & 9.6566 & 1.37674 & 5.55704 \\
$\mathrm{H}$ & 9.70069 & 2.81929 & 4.5513 \\
$\mathrm{C}$ & 9.84809 & 0.85806 & 8.37128 \\
$\mathrm{H}$ & 9.10125 & 0.56218 & 7.62863 \\
$\mathrm{C}$ & 11.22586 & 0.34872 & 7.9389 \\
$\mathrm{H}$ & 11.523 & 0.71732 & 6.95625 \\
$\mathrm{H}$ & 11.21027 & -0.74306 & 7.8869 \\
$\mathrm{H}$ & 11.9986 & 0.63261 & 8.65592 \\
$\mathrm{C}$ & 9.42848 & 0.25604 & 9.71628 \\
$\mathrm{H}$ & 10.11079 & 0.53973 & 10.51932 \\
$\mathrm{H}$ & 9.4398 & -0.83437 & 9.64409 \\
$\mathrm{H}$ & 8.42003 & 0.56014 & 9.99726 \\
$\mathrm{C}$ & 8.48231 & 5.66869 & 11.52742 \\
$\mathrm{H}$ & 9.39649 & 6.04347 & 11.06592 \\
$\mathrm{H}$ & 8.62109 & 5.65752 & 12.6104 \\
$\mathrm{H}$ & 7.65318 & 6.32156 & 11.27072 \\
\hline & & & \\
\hline
\end{tabular}




\section{D.5. Wavefunction analysis}

Table S16. IBO partial charge distributions for all metal-ligand bonds and oxidation state (OS) assignments based on LOBA for compounds 4-6 and $\mathbf{4}^{\prime}-\mathbf{6}^{\prime}$.

\begin{tabular}{|c|c|c|c|c|c|c|}
\hline Compounds & $\mathbf{P}^{\text {NNN }}$ & PiPr2Ar & Cl & Me & N & $\begin{array}{c}\text { OS of } \\
\text { M } \\
\text { (LOBA) }\end{array}$ \\
\hline $\mathbf{4}$ & $\mathrm{Pt}(0.415)$ & $\mathrm{Pt}(0.233)$ & $\mathrm{Pt}(0.237)$ & $\mathrm{Pt}(0.747)$ & - & $+\mathrm{II}$ \\
& $\mathrm{P}(1.516)$ & $\mathrm{P}(1.636)$ & $\mathrm{Cl}(1.694)$ & $\mathrm{C}(1.212)$ & & \\
\hline $\mathbf{5}$ & $\mathrm{Pd}(0.378)$ & $\mathrm{Pd}(0.203)$ & $\mathrm{Pd}(0.233)$ & $\mathrm{Pd}(0.903)$ & - & $+\mathrm{II}$ \\
& $\mathrm{P}(1.533)$ & $\mathrm{P}(1.636)$ & $\mathrm{Cl}(1.694)$ & $\mathrm{C}(1.066)$ & & \\
\hline $\mathbf{6}$ & $\mathrm{Ni}(0.381)$ & $\mathrm{Ni}(0.230)$ & $\mathrm{Ni}(0.252)$ & $\mathrm{Ni}(0.075)$ & - & $+\mathrm{II}$ \\
& $\mathrm{P}(1.545)$ & $\mathrm{P}(1.634)$ & $\mathrm{Cl}(1.689)$ & $\mathrm{C}(1.156)$ & & \\
\hline $\mathbf{4}^{\prime}$ & $\mathrm{Pt}(0.656)$ & $\mathrm{Pt}(0.452)$ & $\mathrm{Pt}(0.186)$ & - & $\mathrm{Pt}(0.271)$ & $+\mathrm{II}$ \\
& $\mathrm{P}(1.299)$ & $\mathrm{P}(1.479)$ & $\mathrm{Cl}(1.741)$ & & $\mathrm{N}(1.544)$ & \\
\hline $\mathbf{5}^{\prime}$ & $\mathrm{Pd}(0.815)$ & $\mathrm{Pd}(0.453)$ & $\mathrm{Pd}(0.168)$ & - & $\mathrm{Pd}(0.150)$ & $+\mathrm{II}$ \\
& $\mathrm{P}(1.154)$ & $\mathrm{P}(1.463)$ & $\mathrm{Cl}(1.748)$ & & $\mathrm{N}(1.620)$ & \\
\hline $\mathbf{6}^{\prime}$ & $\mathrm{Ni}(0.595)$ & $\mathrm{Ni}(0.394)$ & $\mathrm{Ni}(0.221)$ & - & $\mathrm{Ni}(0.279)$ & $+\mathrm{II}$ \\
& $\mathrm{P}(1.357)$ & $\mathrm{P}(1.546)$ & $\mathrm{Cl}(1.720)$ & & $\mathrm{N}(1.554)$ & \\
\hline
\end{tabular}

Table S17. IBO partial charge distributions of the M-P1-N1 cycle for compounds $5^{\prime}$ and $6^{\prime}$.

\begin{tabular}{|c|c|c|c|c|}
\hline Compounds & M-P1 & M-N1 & P1-N1 & P1-N3 \\
\hline $\mathbf{5}^{\prime}$ & $\mathrm{Pd}(0.815)$ & $\mathrm{Pd}(0.150)$ & $\mathrm{P}(0.347)$ & $\mathrm{P}(0.494)$ \\
& $\mathrm{P}(1.154)$ & $\mathrm{N}(1.620)$ & $\mathrm{N}(1.487)$ & $\mathrm{N}(1.416)$ \\
\hline $\mathbf{6}^{\prime}$ & $\mathrm{Ni}(0.595)$ & $\mathrm{Ni}(0.279)$ & $\mathrm{P}(0.257)$ & $\mathrm{P}(0.634)$ \\
& $\mathrm{P}(1.357)$ & $\mathrm{N}(1.554)$ & $\mathrm{N}(1.560)$ & $\mathrm{N}(1.331)$ \\
\hline
\end{tabular}


(a)

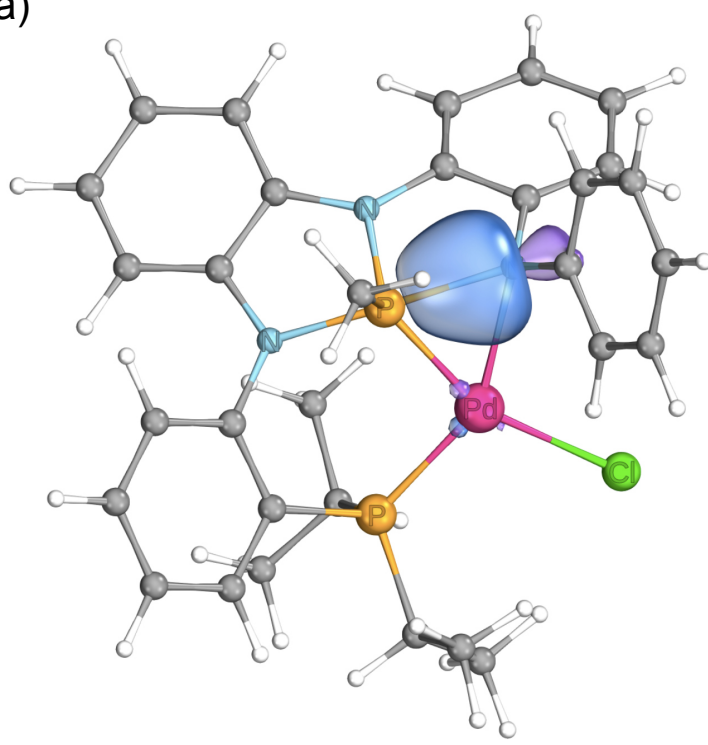

(b)



Figure S30. IBO depictions of P1-N1 bond for compounds (a) $\mathbf{5}^{\prime}$ and (b) $\mathbf{6}^{\prime}$. Numbers in parentheses correspond to the partial charge distributions of IBO. Orbital isosurface contains $80 \%$ of the integrated electron density of the orbital.

\section{D.6. QTAIM analysis}

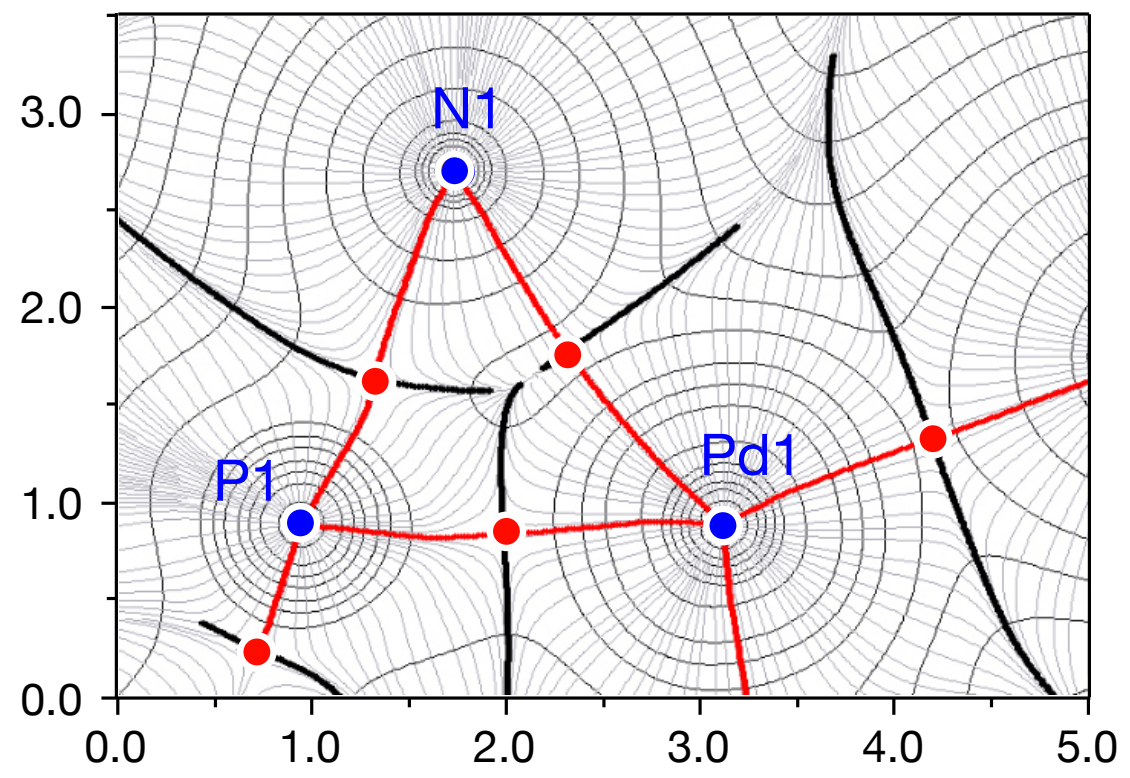

Figure S31. Calculated electron density for $\mathbf{5}^{\prime}$ in the plane defined by $\mathrm{Pd}-\mathrm{P}-\mathrm{N}$ coordinates. Electron density contours (-), gradient vector field (gray -), bond paths (red -), bond critical points (red $\bullet$ ), and atomic basins (bold - ) are depicted. Units are $\AA$. 


\section{E. References}

(1) Ren, P.; Vechorkin, O.; von Allmen, K.; Scopelliti, R.; Hu, X. J. Am. Chem. Soc. 2011, 133, 7084-7095.

(2) Clark, H. C.; Manzer, L. E. J. Organomet. Chem. 1973, 59, 411-428.

(3) Beck, R.; Klein, H.-F. Z. Anorg. Allg. Chem. 2008, 634, 1971-1974.

(4) Fulmer, G. R.; Miller, A. J. M.; Sherden, N. H.; Gottlieb, H. E.; Nudelman, A.; Stoltz, B. M.; Bercaw, J. E.; Goldberg, K. I. Organometallics, 2010, 29, 2176.

(5) Bruker AXS (2009). Apex II. Bruker AXS, Madison, Wisconsin.

(6) Sheldrick, G. M. Acta Cryst. 2010, D66, 479-485.

(7) (a) Ramos-Cordoba, E.; Postils, V.; Salvador, P. Oxidation States from Wave Function Analysis. J. Chem. Theory Comput. 2015, 11 (4), 1501-1508. (b) Postils, V.; Delgado-Alonso, C.; Luis, J. M.; Salvador, P. An Objective Alternative to IUPAC's Approach To Assign Oxidation States. Angew. Chem. Int. Ed. 2018, 57 (33), 10525-10529. (c) Gimferrer, M.; Salvador, P.; Poater, A. Computational Monitoring of Oxidation States in Olefin Metathesis. Organometallics 2019, 38 (24), 4585-4592.

(8) (a) Knizia, G.; Klein, J. E. M. N. Angew. Chem. Int. Ed. 2015, 54, 5518-5522; (b) Knizia, G. http://www.iboview.org/.

(9) (a) Lu, T.; Chen, F. Multiwfn: A Multifunctional Wavefunction Analyzer. J. Comput. Chem. 2012, 33 (5), 580-592. (b) T. Lu, F. Chen, Acta Chim. Sin. 2011, 69, 2393 - 2406.

(10) Pipek, J.; Mezey, P. G. A fast intrinsic localization procedure applicable for ab-initio and semiempirical linear combination of atomic orbital wave functions. J. Chem. Phys. 1989, 90, 4916-4926.

(11) R. F. W. Bader, 'Atoms in Molecules A Quantum Theory', Oxford University Press, Oxford, 1990.

(12) (a) van Lenthe, E.; Baerends, E. J.; Snijders, J. G. J. Chem. Phys. 1993, 99, 4597-4610. (b) van Lenthe, E.; Baerends, E. J.; Snijders, J. G. J. Chem. Phys. 1994, 101, 9783-9792. 